Supporting Information for

\title{
Catalytic Enantioselective 1,3-Dipolar Cycloaddition of Azomethine Ylides with Vinyl Sulfones
}

\author{
Tomás Llamas, Ramón Gómez Arrayás, and Juan C. Carretero* \\ Departamento de Química Orgánica. Facultad de Ciencias. Universidad Autónoma de Madrid. Cantoblanco. \\ 28049 Madrid. Spain.
}

Contents

1. Experimental Section

S 2

2. NMR Spectra

$\mathrm{S} 12$

3. X-Ray data of exo-3a

S 43 


\section{General methods}

All the reactions were carried out in anhydrous solvents and under argon atmosphere. Melting points were taken in open-end capillary tubes. NMR spectra were recorded at 200 $\mathrm{MHz}\left({ }^{1} \mathrm{H}\right), 50 \mathrm{MHz}\left({ }^{13} \mathrm{C}\right)$ or at $300 \mathrm{MHz}\left({ }^{1} \mathrm{H}\right), 75 \mathrm{MHz}\left({ }^{13} \mathrm{C}\right)$, at room temperature in $\mathrm{CDCl}_{3}$ [calibrated at $7.26 \mathrm{ppm}\left({ }^{1} \mathrm{H}\right)$, and $77.0 \mathrm{ppm}\left({ }^{13} \mathrm{C}\right)$ ]. Optical rotation was obtained on a Perkin-Elmer 241 polarimeter. MS spetra were recorded on a VG AutoSpec mass spectrometer. HPLC experiments were conducted using Daicel Chiralpak AS-H column. Flash column chromatography was performed using silica gel Merk-60 (230-400 mesh). Methyl (E)-N-benzylideneglycinate (1a) and phenyl vinyl sulfone (2a) were purchased from commercial sources.

Typical procedure for the synthesis of . $\alpha$-imino esters: methyl $(E)-N-[(p-$ fluorophenyl)methylene]glycinate (1b). To a suspension of methyl glycinate hydrochloride $(1.37 \mathrm{~g}, 10.9 \mathrm{mmol})$ and $\mathrm{MgSO}_{4}(2.38 \mathrm{~g}, 19.8 \mathrm{mmol})$ in $\mathrm{CH}_{2} \mathrm{Cl}_{2}(10 \mathrm{~mL})$ was added $\mathrm{Et}_{3} \mathrm{~N}$ (1.5 mL, $\left.10.9 \mathrm{mmol}\right)$. The mixture was stirred at room temperature for $1 \mathrm{~h}$, then $p$-fluorobenzaldehyde $(1.1 \mathrm{~mL}, 9.9 \mathrm{mmol})$ was added. The reaction mixture was stirred at room temperature for $17 \mathrm{~h}$ and it was washed with water $(2 \times 5 \mathrm{~mL})$ and brine $(5 \mathrm{~mL})$. The combined organic phase was dried $\left(\mathrm{MgSO}_{4}\right)$ and concentrated to afford $\mathbf{1 b}$ as a yellow oil; yield: $1.08 \mathrm{~g}(56 \%)$. This material was pure by NMR and used in 1,3-dipolar reactions without further purification. ${ }^{1} \mathrm{H}$ NMR $(300 \mathrm{MHz}): \delta 8.25(\mathrm{~s}, 1 \mathrm{H}), 7.81-7.70(\mathrm{~m}, 2 \mathrm{H}), 7.15-$ $7.02(\mathrm{~m}, 2 \mathrm{H}), 4.40(\mathrm{~s}, 2 \mathrm{H}), 3.77(\mathrm{~s}, 3 \mathrm{H}) .{ }^{13} \mathrm{C} \mathrm{NMR}(75 \mathrm{MHz}): \delta 170.5,164.6\left(\mathrm{~d}, J_{\mathrm{F}-\mathrm{C}}=251.4\right.$ $\mathrm{Hz}) 163.9,131.6\left(\mathrm{~d}, J_{\mathrm{F}-\mathrm{C}}=3.3 \mathrm{~Hz}\right), 130.4\left(\mathrm{~d}, J_{\mathrm{F}-\mathrm{C}}=8.8 \mathrm{~Hz}\right), 115.7\left(\mathrm{~d}, J_{\mathrm{F}-\mathrm{C}}=21.9 \mathrm{~Hz}\right), 61.8$, 52.1 .

Methyl $(E)-N$-[(p-methoxyphenyl)methylene]glycinate (1c). White solid; yield: $1.48 \mathrm{~g}$ (72\%); mp 69-70 ${ }^{\circ} \mathrm{C} .{ }^{1} \mathrm{H}$ NMR $(300 \mathrm{MHz}): \delta 8.16(\mathrm{~s}, 1 \mathrm{H}), 7.68(\mathrm{~d}, J=8.7,2 \mathrm{H}), 6.89(\mathrm{~d}, J=$ 8.7, 2H), 4.34 (s, 2H), 3.79 (s, 3H), 3.73 (s, 3H). ${ }^{13} \mathrm{C}$ NMR (75 MHz) $\delta 170.6,164.5,161.9$, $130.0,128.4,113.8,61.8,55.2,51.9$.

Methyl $(E)-N-[(m$-fluorophenyl)methylene $]$ glycinate (1d). Brown oil; yield: $1.70 \mathrm{~g}$ (88\%). ${ }^{1} \mathrm{H}$ NMR $(300 \mathrm{MHz}): \delta 8.27(\mathrm{~s}, 1 \mathrm{H}), 7.58-7.47(\mathrm{~m}, 1 \mathrm{H}), 7.45-7.30(\mathrm{~m}, 2 \mathrm{H}), 7.21-$ $7.07(\mathrm{~m}, 1 \mathrm{H}), 4.43(\mathrm{~s}, 2 \mathrm{H}), 3.79(\mathrm{~s}, 3 \mathrm{H})$.

Methyl $(E)-N$-[(m-methoxyphenyl)methylene $]$ glycinate (1e). Yellow oil; yield: $1.42 \mathrm{~g}$ (69\%). ${ }^{1} \mathrm{H}$ NMR (300 MHz): $\delta 8.27(\mathrm{~s}, 1 \mathrm{H}), 7.42-7.45(\mathrm{~m}, 3 \mathrm{H}), 7.04-6.97(\mathrm{~m}, 1 \mathrm{H}), 4.42(\mathrm{~s}$, 2H), $3.85(\mathrm{~s}, 3 \mathrm{H}), 3.78(\mathrm{~s}, 3 \mathrm{H}) .{ }^{13} \mathrm{C}$ NMR $(75 \mathrm{MHz}): \delta 170.3,165.2,159.7,136.8,129.4$, 121.7, 117.9, 111.5, 61.7, 55.2, 51.9.

Methyl $(\boldsymbol{E})-\boldsymbol{N}$-[(m-tolyl)methylene]glycinate (1f). Orange oil; yield: $1.74 \mathrm{~g}(92 \%) .{ }^{1} \mathrm{H}$ NMR (300 MHz): $\delta 8.27(\mathrm{~s}, 1 \mathrm{H}), 7.66(\mathrm{~s}, 1 \mathrm{H}), 7.58-7.49(\mathrm{~m}, 1 \mathrm{H}), 7.36-7.20(\mathrm{~m}, 2 \mathrm{H}), 4.43$ (s, 2H), 3.79 (s, 3H), 2.40 (s, 3H). ${ }^{13} \mathrm{C}$ NMR (75 MHz): $\delta 170.4,165.5,138.2,135.3,131.9$, $128.4,128.3,126.0,61.8,51.9,21.1$.

Methyl (E)- $N$-[(2-Naphtyl)methylene]glycinate (1g). White solid; yield: $1.89 \mathrm{~g}(84 \%)$; mp 89-90 ${ }^{\circ} \mathrm{C} .{ }^{1} \mathrm{H}$ NMR (300 MHz): $\delta 8.41(\mathrm{~s}, 1 \mathrm{H}), 8.09-7.99(\mathrm{~m}, 2 \mathrm{H}), 7.92-7.80(\mathrm{~m}, 3 \mathrm{H})$, 7.57-7.46 (m, 2H), 4.47 (s, 2H), $3.79(\mathrm{~s}, 3 \mathrm{H}) .{ }^{13} \mathrm{C} \mathrm{NMR}(75 \mathrm{MHz}): \delta 170.5,165.4,134.9$, $133.1,132.9,130.5,128.6,128.4,127.8,127.3,126.4,123.8,62.0,52.1$. 
Methyl (E)-N-[(o-tolyl)methylene]glycinate (1h). White solid; yield: $1.51 \mathrm{~g}(80 \%)$; mp 47-48 ${ }^{\circ} \mathrm{C} .{ }^{1} \mathrm{H}$ NMR $(300 \mathrm{MHz}): \delta 8.60(\mathrm{~s}, 1 \mathrm{H}), 7.96-7.89(\mathrm{~m}, 1 \mathrm{H}), 7.37-7.12(\mathrm{~m}, 3 \mathrm{H}), 4.44$ (s, 2H), 3.79 (s, 3H), $2.52(\mathrm{~s}, 3 \mathrm{H}) .{ }^{13} \mathrm{C}$ NMR (75 MHz): $\delta$ 170.6, 164.0, 137.9, 133.5, 130.7, 127.7, 126.1, 62.3, 52.0, 19.7.

Methyl $(E)-N$-(cyclohexylmethylene)glycinate (1i). Colourless oil; yield: $1.09 \mathrm{~g}(60 \%)$. ${ }^{1} \mathrm{H}$ NMR $(300 \mathrm{MHz}): \delta$ 7.55-7.51 (m, 1H), $4.14(\mathrm{~s}, 2 \mathrm{H}), 3.73(\mathrm{~s}, 3 \mathrm{H}), 1.92-1.54(\mathrm{~m}, 6 \mathrm{H})$, 1.40-1.05 (m, 5H).

Methyl (E)- $\boldsymbol{N}$-benzylidenealaninate (1k). Colourless oil; yield: $1.32 \mathrm{~g}(70 \%) .{ }^{1} \mathrm{H}$ NMR $(300 \mathrm{MHz}): \delta 8.04(\mathrm{~s}, 1 \mathrm{H}), 7.56-7.46(\mathrm{~m}, 2 \mathrm{H}), 7.22-6.95(\mathrm{~m}, 3 \mathrm{H}), 3.89(\mathrm{c}, J=6.8 \mathrm{~Hz}, 1 \mathrm{H})$, $3.46(\mathrm{~s}, 3 \mathrm{H}), 1.27(\mathrm{~d}, J=6.8 \mathrm{~Hz}, 3 \mathrm{H}) .{ }^{13} \mathrm{C} \mathrm{NMR}(75 \mathrm{MHz}): \delta$ 172.6, 162.6, 135.4, 130.8, $128.2,128.1,67.6,51.8,19.1$.

Typical procedure for the synthesis of vinyl sulfones 2b-d: 2-naphthyl vinyl sulfone (2b). To a solution of 2-naphthyldisulfide (1.59 g, $5.0 \mathrm{mmol})$ in THF (50 mL), cooled to $78^{\circ} \mathrm{C}$, was added a $1 \mathrm{M}$ solution of vinyl magnesium bromide in THF (7.5 mL, $7.5 \mathrm{mmol}$ ). The mixture was stirred for $1 \mathrm{~h}$ at $-78{ }^{\circ} \mathrm{C}$, then a sat. aq solution of $\mathrm{NH}_{4} \mathrm{Cl}(2 \mathrm{~mL})$ was added. The organic phase was separated and washed with a $2 \mathrm{~N}$ aq solution of $\mathrm{NaOH}(2 \mathrm{x}$ $10 \mathrm{~mL})$. The combined organic phase was dried $\left(\mathrm{MgSO}_{4}\right)$ and concentrated. The residue was dissolved in EtOAc $(25 \mathrm{~mL})$ and treated with $\mathrm{H}_{2} \mathrm{O}(3 \mathrm{~mL})$ and $\mathrm{Na}_{2} \mathrm{~W}_{7} \mathrm{O}_{4} \cdot 2 \mathrm{H}_{2} \mathrm{O}(131$ $\mathrm{mg}, 0.25 \mathrm{mmol})$. To the resulting mixture, cooled to $0{ }^{\circ} \mathrm{C}$, was slowly added $30 \% \mathrm{H}_{2} \mathrm{O}_{2}$ $(0.75 \mathrm{~mL})$. The mixture was stirred at $0{ }^{\circ} \mathrm{C}$ for $30 \mathrm{~min}$, then it was allowed to reach $\mathrm{rt}$ and stirred at $\mathrm{rt}$ for further $5 \mathrm{~h}$. Then it was cooled again to $0{ }^{\circ} \mathrm{C}$ and treated with a $1 \mathrm{M}$ aq solution of $\mathrm{Na}_{2} \mathrm{SO}_{3}(6 \mathrm{~mL})$. The aqueous phase was extracted with EtOAc $(2 \times 10 \mathrm{~mL})$ and the combined organic phase was dried $\left(\mathrm{MgSO}_{4}\right)$ and concentrated. The residue was purified by flash chromathography ( $n$-hexane-EtOAc $4: 1$ ) to afford $\mathbf{2 b}$ as a white solid; yield: 764 $\mathrm{mg}(70 \%)$; mp 97-98 ${ }^{\circ} \mathrm{C} .{ }^{1} \mathrm{H}$ NMR $(300 \mathrm{MHz}): \delta 8.50(\mathrm{~s}, 1 \mathrm{H}), 8.05-7.87(\mathrm{~m}, 3 \mathrm{H}), 7.86-$ $7.77(\mathrm{~m}, 1 \mathrm{H}), 7.72-7.57(\mathrm{~m}, 2 \mathrm{H}), 6.72(\mathrm{dd}, J=16.6,9.8 \mathrm{~Hz}, 1 \mathrm{H}), 6.51(\mathrm{~d}, J=16.6 \mathrm{~Hz}, 1 \mathrm{H})$, $6.07(\mathrm{~d}, J=9.8 \mathrm{~Hz}, 1 \mathrm{H}) .{ }^{13} \mathrm{C}$ NMR $(75 \mathrm{MHz}): \delta 138.5,136.4,135.3,132.2,129.7,129.7$, $129.4,129.3,128.0,127.8,127.7,122.6$.

2-Thienyl vinyl sulfone (2c). Chromatography: $n$-hexane-EtOAc 4:1. White solid; yield: $662 \mathrm{mg}(76 \%) ; \mathrm{mp} \mathrm{47-48}{ }^{\circ} \mathrm{C} .{ }^{1} \mathrm{H}$ NMR $(300 \mathrm{MHz}): \delta 7.72(\mathrm{dd}, J=4.9,1.3 \mathrm{~Hz}, 1 \mathrm{H}), 7.69$ (dd, $J=3.8,1.3 \mathrm{~Hz}, 1 \mathrm{H}), 7.15(\mathrm{dd}, J=4.9,3.8 \mathrm{~Hz}, 1 \mathrm{H}), 6.76(\mathrm{dd}, J=16.5,9.8 \mathrm{~Hz}, 1 \mathrm{H}), 6.46$ $(\mathrm{dd}, J=16.5,0.5 \mathrm{~Hz}, 1 \mathrm{H}), 6.46(\mathrm{dd}, J=9.8,0.5 \mathrm{~Hz}, 1 \mathrm{H}) .{ }^{13} \mathrm{C} \mathrm{NMR}(75 \mathrm{MHz}): \delta 140.8$, 139.0, 134.4, 134.1, 128.1, 127.3. FAB MS m/z $175.0\left(\mathrm{M}^{+}+\mathrm{H}, 29\right)$.

2-Pyridyl vinyl sulfone (2d). Chromatography: $n$-hexane-EtOAc 2:1. Yellow oil; yield: $728 \mathrm{mg}(86 \%) .{ }^{1} \mathrm{H}$ NMR $(200 \mathrm{MHz}): \delta 8.75-8.65(\mathrm{~m}, 1 \mathrm{H}), 8.10-7.86(\mathrm{~m}, 2 \mathrm{H}), 7.58-7.46$ $(\mathrm{m}, 1 \mathrm{H}), 6.87(\mathrm{dd}, J=16.7,10.2 \mathrm{~Hz}, 1 \mathrm{H}), 6.54(\mathrm{~d}, J=16.7 \mathrm{~Hz}, 1 \mathrm{H}), 6.18(\mathrm{~d}, J=10.2 \mathrm{~Hz}$, 1H). ${ }^{13} \mathrm{C}$ NMR (50 MHz): $\delta 157.5,150.3,138.4,135.7,130.7,127.4,122.1 . \mathrm{FAB} \mathrm{MS} \mathrm{m} / \mathrm{z}$ $170.1\left(\mathrm{M}^{+}+\mathrm{H}, 100\right)$. 
Preparation of $o$-(N,N-dimethylamino)phenyl vinyl sulfone (2e).

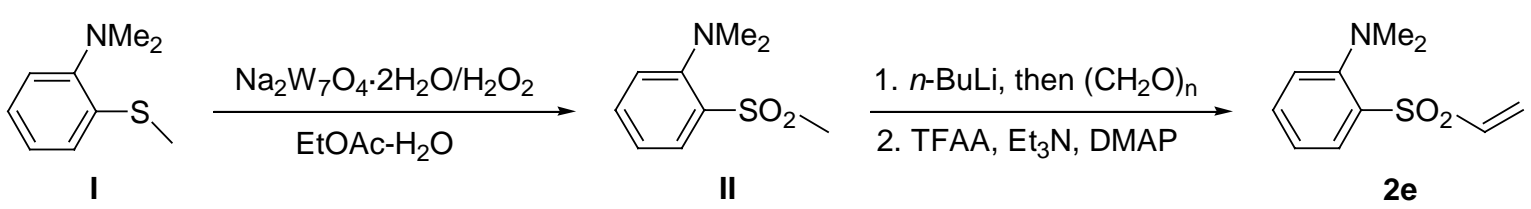

$\boldsymbol{o}$-(N,N-dimethylamino)phenyl methyl sulfone (II). ${ }^{1}$ To a solution of $o-(N, N-$ dimethylamino)phenyl methyl tioether $(\mathbf{I})(836 \mathrm{mg}, 5.0 \mathrm{mmol})$ in EtOAc $(50 \mathrm{~mL})$ and $\mathrm{H}_{2} \mathrm{O}$ $(6 \mathrm{~mL})$, cooled to $0{ }^{\circ} \mathrm{C}$, were successively added $\mathrm{Na}_{2} \mathrm{~W}_{7} \mathrm{O}_{4} \cdot 2 \mathrm{H}_{2} \mathrm{O}(262 \mathrm{mg}, 0.5 \mathrm{mmol})$ and $30 \% \mathrm{H}_{2} \mathrm{O}_{2}(1.5 \mathrm{~mL})$. The mixture was stirred at $0{ }^{\circ} \mathrm{C}$ for $30 \mathrm{~min}$, then it was allowed to reach $\mathrm{rt}$ and stirred at $\mathrm{rt}$ for further $5 \mathrm{~h}$. The mixture was cooled again to $0{ }^{\circ} \mathrm{C}$ and treated with a $1 \mathrm{M}$ aq solution of $\mathrm{Na}_{2} \mathrm{SO}_{3}(12 \mathrm{~mL})$. The aqueous phase was extracted with AcOEt $(2 \times 20 \mathrm{~mL})$ and the combined organic phase was dried $\left(\mathrm{MgSO}_{4}\right)$ and concentrated. The residue was purified by flash chromathography ( $n$-hexane-EtOAc 5:1) to afford II as a yellow solid; yield: $737 \mathrm{mg}(74 \%)$; mp 94-95 ${ }^{\circ} \mathrm{C} .{ }^{1} \mathrm{H}$ NMR $(300 \mathrm{MHz}): \delta 8.05(\mathrm{~m}, 1 \mathrm{H})$, $7.61(\mathrm{~m}, 1 \mathrm{H}), 7.42(\mathrm{~m} .1 \mathrm{H}), 7.31(\mathrm{~m} .1 \mathrm{H}), 3.22\left(\mathrm{~s}, 3 \mathrm{H}, \mathrm{CH}_{3}\right), 2.80\left(\mathrm{~s}, 6 \mathrm{H},\left(\mathrm{CH}_{3}\right)_{2} \mathrm{~N}\right) .{ }^{13} \mathrm{C}$ NMR (75 MHz): $\delta 153.8,136.5,134.5,129.2,124.9,123.4,46.0,42.3$. EI+ MS m/z 199 $\left(\mathrm{M}^{+}, 34\right), 184\left(\mathrm{M}^{+}-\mathrm{CH}_{3}, 35\right), 118$ (73), 91 (100). HMRS (FAB) Calcd for $\mathrm{C}_{9} \mathrm{H}_{14} \mathrm{NO}_{2} \mathrm{~S}$, 200.0745; found, 200.0747.

$\boldsymbol{o}$-( $\mathrm{N}, \mathrm{N}$-dimethylamino)phenyl vinyl sulfone (2e). To a solution of II (500 mg, $2.5 \mathrm{mmol})$ in THF $(20 \mathrm{~mL})$, cooled to $-78^{\circ} \mathrm{C}$, was slowly added a $2.5 \mathrm{M}$ solution of $n$-BuLi in hexanes $(1.2 \mathrm{~mL}, 3.0 \mathrm{mmol})$. The mixture was stirred at $-78^{\circ} \mathrm{C}$ for $30 \mathrm{~min}$, then it was warmed to 0 ${ }^{\circ} \mathrm{C}$ and a large excess of fresly sublimed $\left(\mathrm{CH}_{2} \mathrm{O}\right)_{\mathrm{n}}(1.50 \mathrm{~g}, 50 \mathrm{mmol})$ was added via cannula. The mixture was stirred at $0{ }^{\circ} \mathrm{C}$ for $1 \mathrm{~h}$ and $\mathrm{H}_{2} \mathrm{O}(2 \mathrm{~mL})$ was added. The mixture was washed with brine $(5 \mathrm{~mL})$ and the combined organic phase was dried $\left(\mathrm{MgSO}_{4}\right)$ and concentrated. The residue was purified by flash chromathography ( $n$-hexane-EtOAc 2:1) to afford the intermediate alcohol as a colourless oil; yield: $190 \mathrm{mg}(33 \%)$ along with starting material (226 mg, 45\%). To a solution of the alcohol (190 mg, $0.82 \mathrm{mmol})$ and DMAP (10 $\mathrm{mg}, 0.082 \mathrm{mmol}$ ) in $\mathrm{CH}_{2} \mathrm{Cl}_{2}(5 \mathrm{~mL})$, cooled to $0{ }^{\circ} \mathrm{C}$, were successively added $\mathrm{Et}_{3} \mathrm{~N}(230 \mu \mathrm{L}$, $1.64 \mathrm{mmol})$ and TFAA $(0.14 \mathrm{~mL}, 0.98 \mathrm{mmol})$. The mixture was stirred at $\mathrm{rt}$ overnight, and then sat. aq solution of $\mathrm{NH}_{4} \mathrm{Cl}(1 \mathrm{~mL})$ was added. The mixture was washed with brine (2 $\mathrm{mL}$ ), and the combined organic phase was dried $\left(\mathrm{MgSO}_{4}\right)$ and concentrated. The residue was purified by flash cromathography ( $n$-hexane-EtOAc 2:1) to afford $2 \mathbf{e}$ as a colourless oil; yield: $137 \mathrm{mg}(79 \%) .{ }^{1} \mathrm{H}$ NMR $(300 \mathrm{MHz}): \delta$ 7.99-7.94 $(\mathrm{m}, 1 \mathrm{H}), 7.55-7.47(\mathrm{~m}, 1 \mathrm{H})$, 7.34-7.29 (m, 1H), 7.25-7.18 (m, 1H), $7.09(\mathrm{dd}, J=16.8,9.9 \mathrm{~Hz}, 1 \mathrm{H}), 6.32(\mathrm{~d}, J=16.8 \mathrm{~Hz}$, $1 \mathrm{H}), 5.93(\mathrm{~d}, J=9.9 \mathrm{~Hz}, 1 \mathrm{H}), 2.67(\mathrm{~s}, 6 \mathrm{H}) .{ }^{13} \mathrm{C} \mathrm{NMR}(75 \mathrm{MHz}): \delta 154.1,138.7,137.0$, 134.6, 129.3, 127.4, 125.2, 123.7, 45.9. FAB MS m/z $212.1\left(\mathrm{M}^{+}+\mathrm{H}, 100\right)$.

\footnotetext{
${ }^{1}$ Mauleón, P; Nuñez, A. A.; Alonso, I.; Carretero, J. C. Chem. Eur. J. 2003, 9, 1511.
} 


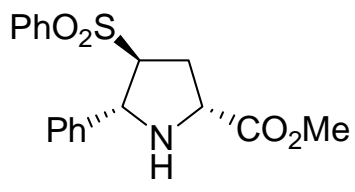

Typical procedure for asymmetric 1,3-dipolar cycloaddition of azomethine ylides: Methyl $(2 R, 4 S, 5 R)-5$-phenyl-4-phenylsulfonylpyrrolidine-2-carboxylate (exo-3a). A solution of Taniaphos ligand $(6.9 \mathrm{mg}, 0.01 \mathrm{mmol})$ and $\mathrm{Cu}\left(\mathrm{CH}_{3} \mathrm{CN}\right)_{4} \mathrm{ClO}_{4}(3.2 \mathrm{mg}, 0.01$ $\mathrm{mmol})$ in toluene $(1 \mathrm{~mL})$ was stirred at room temperature for $30 \mathrm{~min}$. Then a solution of phenylmethylene glycinate $(35.6 \mathrm{mg}, 0.20 \mathrm{mmol})$ in toluene $(0.5 \mathrm{~mL})$ and $\mathrm{Et}_{3} \mathrm{~N}(5.0 \mu \mathrm{L}$, $0.036 \mathrm{mmol}$ ) were successively added. The mixture was cooled to $0{ }^{\circ} \mathrm{C}$ and a solution of phenyl vinyl sulfone $(33.6 \mathrm{mg}, 0.20 \mathrm{mmol})$ in toluene $(0.5 \mathrm{~mL})$ was added. After $24 \mathrm{~h}$ the mixture was filtered through Celite and concentrated. The residue was purified by flash chromatography ( $n$-hexane-EtOAc 3:1) 3a to afford as a white solid; yield: $60 \mathrm{mg}$ (87\%); mp 101-102 ${ }^{\circ} \mathrm{C} .[\alpha]_{\mathrm{D}}{ }^{20}=-14\left(\mathrm{c} 0.50, \mathrm{CHCl}_{3}\right), 83 \%$ ee. HPLC: Daicel Chiralpak AS-H, iPrOH-hexane 40/60, flow rate $0.8 \mathrm{~mL} / \mathrm{min}, t_{\mathrm{R}}: 28.7 \mathrm{~min}(2 R, 4 S, 5 R)$-isomer and $32.3 \mathrm{~min}$ $(2 S, 4 R, 5 S)$-isomer, $210 \mathrm{~nm}$. Recrystalyzation with dichloromethane $/ n$-hexane at $-20{ }^{\circ} \mathrm{C}$ affored $(2 R, 4 S, 5 R)$-isomer; $[\alpha]_{\mathrm{D}}{ }^{20}=-21\left(\mathrm{c} 0.50, \mathrm{CHCl}_{3}\right), 99 \%$ ee. ${ }^{1} \mathrm{H}$ NMR $(300 \mathrm{MHz}): \delta$ 7.85-7.77 (m, 2H), 7.63-7.56 (m, 1H), 7.52-7.44 (m, 2H), 7.21 (bs, 5H), 4.73 (d, J=5.4 Hz, $1 \mathrm{H}), 4.18(\mathrm{t}, J=7.9 \mathrm{~Hz}, 1 \mathrm{H}), 3.77(\mathrm{~s}, 3 \mathrm{H}), 3.66(\mathrm{~m}, 1 \mathrm{H}), 2.70(\mathrm{ddd}, J=14.0,7.5,4.4 \mathrm{~Hz}, 1 \mathrm{H}$ ), $2.56(\mathrm{bs}, 1 \mathrm{H}), 2.36(\mathrm{dt}, J=14.0,8.9 \mathrm{~Hz}, 1 \mathrm{H}) .{ }^{13} \mathrm{C} \mathrm{NMR}(75 \mathrm{MHz}): \delta 173.2,141.0,138.0$, 133.8, 129.2, 128.5, 128.5, 127.8, 126.8, 70.1, 62.7, 59.3, 52.3, 31.1. IE MS m/z 346.0 $\left(\mathrm{M}^{+}+\mathrm{H}, 100\right)$. HRMS (EI) Calcd for $\mathrm{C}_{18} \mathrm{H}_{20} \mathrm{NO}_{4} \mathrm{~S}, 346.1121$; found, 346.1115 .

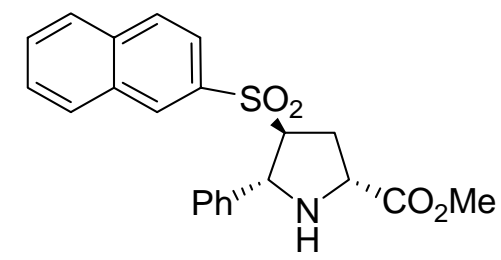

Methyl (2R,4S,5R)-4-(2-naphthyl)sulfonyl-5-phenylpyrrolidine-2-carboxylate (exo-3b). White solid; yield: $56 \mathrm{mg}(71 \%)$; mp $108-109{ }^{\circ} \mathrm{C} .[\alpha]_{\mathrm{D}}{ }^{20}=0 \quad\left(\mathrm{c} 0.51, \mathrm{CHCl}_{3}\right) .{ }^{1} \mathrm{H} \mathrm{NMR}$ (300 MHz): $\delta 8.40(\mathrm{bs}, 1 \mathrm{H}), 7.97-7.84(\mathrm{~m}, 3 \mathrm{H}), 7.76-7.59(\mathrm{~m}, 3 \mathrm{H}), 7.23-7.04(\mathrm{~m}, 5 \mathrm{H}), 4.77$ $(\mathrm{d}, J=5.7 \mathrm{~Hz}, 1 \mathrm{H}), 4.21(\mathrm{t}, J=7.8 \mathrm{~Hz}, 2 \mathrm{H}), 3.80-3.65(\mathrm{~m}, 4 \mathrm{H}), 2.75(\mathrm{ddd}, J=13.9,7.6,4.6$ $\mathrm{Hz}, 1 \mathrm{H}), 2.57(\mathrm{bs}, 1 \mathrm{H}), 2.37(\mathrm{dt}, J=13.9,8.9 \mathrm{~Hz}, 1 \mathrm{H}) .{ }^{13} \mathrm{C} \mathrm{NMR}(75 \mathrm{MHz}): \delta 173.3$, 140.9, 135.3, 134.7, 132.0, 130.5, 129.6, 129.4, 129.3, 128.4, 127.9, 127.7, 127.7, 126.8, 122.8, 70.1, 62.9, 59.3, 52.3, 31.2. FAB+ MS m/z 396.1 $\left(\mathrm{M}^{+}+\mathrm{H}, 100\right)$. HRMS (EI) Calcd for $\mathrm{C}_{22} \mathrm{H}_{22} \mathrm{NO}_{4} \mathrm{~S}, 396.1264$; found, 396.1269.

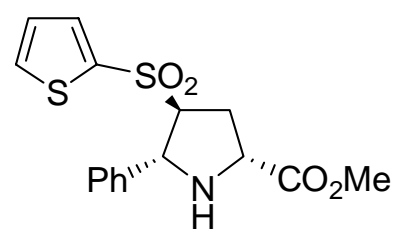

Methyl (2R,4S,5R)-5-phenyl-4-(2-thienyl)sulfonylpyrrolidine-2-carboxylate (exo-3c). White solid; yield: $61 \mathrm{mg}(87 \%)$; mp $84-85^{\circ} \mathrm{C}$. $[\alpha]_{\mathrm{D}}{ }^{20}=-16\left(\mathrm{c} 0.67, \mathrm{CHCl}_{3}\right), 83 \%$ ee. HPLC: Daicel Chiralpak AS-H, i-PrOH-hexane 40/60, flow rate $0.8 \mathrm{~mL} / \mathrm{min}, t_{\mathrm{R}}: 35.1 \mathrm{~min}$ 
$(2 R, 4 S, 5 R)$-isomer and $55.1 \mathrm{~min}(2 S, 4 R, 5 S)$-isomer, $210 \mathrm{~nm} .{ }^{1} \mathrm{H}$ NMR $(300 \mathrm{MHz}): \delta 7.68$ $(\mathrm{dd}, J=5.0,1.1 \mathrm{~Hz}, 1 \mathrm{H}), 7.62(\mathrm{dd}, J=3.7,1.1 \mathrm{~Hz}, 1 \mathrm{H}), 7.26(\mathrm{~m}, 5 \mathrm{H}), 7.09$ (dd, $J=4.8,3.9$ $\mathrm{Hz}, 1 \mathrm{H}), 4.77$ (d, $J=5.4 \mathrm{~Hz}, 1 \mathrm{H}), 4.14(\mathrm{~m}, 1 \mathrm{H}), 3.80-3.64(\mathrm{~m}, 4 \mathrm{H}), 2.76$ (ddd, $J=14.0,7.4$, $4.2 \mathrm{~Hz}, 1 \mathrm{H}), 2.57$ (bs, $1 \mathrm{H}), 2.43$ (dt, $J=14.0,9.0 \mathrm{~Hz}, 1 \mathrm{H}) .{ }^{13} \mathrm{C}$ NMR $(75 \mathrm{MHz}): \delta 173.1$, 141.0, 138.7, 134.9, 134.5, 128.6, 128.0, 127.8, 126.8, 71.7, 63.1, 59.3, 52.3, 31.5. FAB+ MS m/z 352.0 $\left(\mathrm{M}^{+}+\mathrm{H}, 100\right)$. HRMS (EI) Calcd for $\mathrm{C}_{16} \mathrm{H}_{18} \mathrm{NO}_{4} \mathrm{~S}_{2}, 352.0685$; found, 352.0677 .

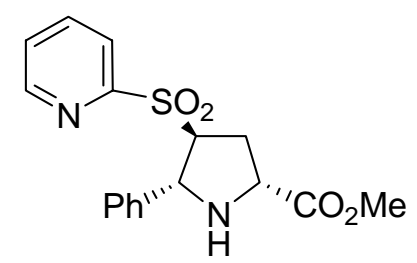

Methyl (2R,4S,5R)-5-phenyl-4-(2-pyridyl)sulfonylpyrrolidine-2-carboxylate (exo-3d). White solid; yield: $50 \mathrm{mg}(72 \%)$; $\mathrm{mp} 103-104{ }^{\circ} \mathrm{C}$. $[\alpha]_{\mathrm{D}}{ }^{20}=+3\left(\mathrm{c} 0.83, \mathrm{CHCl}_{3}\right), 50 \%$ ee. HPLC: Daicel Chiralpak AS-H, i-PrOH-hexane 40/60, flow rate $0.8 \mathrm{~mL} / \mathrm{min}, t_{\mathrm{R}}: 39.9 \mathrm{~min}$ $(2 R, 4 S, 5 R)$-isomer and $63.5 \mathrm{~min}(2 S, 4 R, 5 S)$-isomer, $210 \mathrm{~nm} .{ }^{1} \mathrm{H}$ NMR $(300 \mathrm{MHz}): \delta 8.55-$ $8.45(\mathrm{~m}, 1 \mathrm{H}), 8.05-7.95(\mathrm{~m}, 1 \mathrm{H}), 7.90-7.80(\mathrm{~m}, 1 \mathrm{H}), 7.46-7.38(\mathrm{~m}, 1 \mathrm{H}), 7.27-7.11(\mathrm{~m}, 5 \mathrm{H})$, $4.73(\mathrm{~d}, J=6.1 \mathrm{~Hz}, 1 \mathrm{H}), 4.23(\mathrm{~m}, 2 \mathrm{H}), 3.78(\mathrm{~s}, 3 \mathrm{H}), 2.76(\mathrm{ddd}, J=13.9,7.8,5.1 \mathrm{~Hz}, 1 \mathrm{H})$, 2.60 (bs, $1 \mathrm{H}), 2.47$ (dt, $J=13.8,9.1 \mathrm{~Hz}, 1 \mathrm{H}) .{ }^{13} \mathrm{C}$ NMR $(75 \mathrm{MHz}): \delta 173.3,156.3,150.2$, $140.4,137.8,128.4,127.8,127.3,127.0,122.8,66.1,62.9,59.3,52.3,30.7 . \mathrm{FAB}+\mathrm{MS} \mathrm{m} / \mathrm{z}$ $347.0\left(\mathrm{M}^{+}+\mathrm{H}, 100\right)$.<smiles>CC(=O)[C@@H]1C[C@H](SO)[C@H](c2ccc(F)cc2)N1</smiles>

Methyl (2R,4S,5R)-5-(p-fluorophenyl)-4-phenylsulfonylpyrrolidine-2-carboxylate (4). Yellow solid; yield: $66 \mathrm{mg}(91 \%)$; $\mathrm{mp} 75-76{ }^{\circ} \mathrm{C} .[\alpha]_{\mathrm{D}}{ }^{20}=-22\left(\mathrm{c} 0.50, \mathrm{CHCl}_{3}\right), 82 \%$ ee. HPLC: Daicel Chiralpak AS-H, i-PrOH-hexane 40/60, flow rate $0.8 \mathrm{~mL} / \mathrm{min}, t_{\mathrm{R}}: 32.4 \mathrm{~min}$ $(2 R, 4 S, 5 R)$-isomer and $49.0 \mathrm{~min}(2 S, 4 R, 5 S)$-isomer, $210 \mathrm{~nm} .{ }^{1} \mathrm{H}$ NMR $(300 \mathrm{MHz}): \delta 7.85-$ $7.75(\mathrm{~m}, 2 \mathrm{H}), 7.62(\mathrm{t}, J=7.4 \mathrm{~Hz}, 1 \mathrm{H}), 7.54-7.45(\mathrm{~m}, 2 \mathrm{H}), 7.26-7.15(\mathrm{~m}, 2 \mathrm{H}), 6.90(\mathrm{t}, J=8.6$ $\mathrm{Hz}, 1 \mathrm{H}), 4.73(\mathrm{~d}, J=5.8 \mathrm{~Hz}, 1 \mathrm{H}), 4.16(\mathrm{t}, J=7.9 \mathrm{~Hz}, 1 \mathrm{H}), 3.76(\mathrm{~s}, 3 \mathrm{H}), 3.60(\mathrm{~m}, 1 \mathrm{H}), 2.76$ (ddd, $J=10.3,5.6,4.9 \mathrm{~Hz}, 1 \mathrm{H}), 2.55(\mathrm{bs}, 1 \mathrm{H}), 2.34(\mathrm{dt}, J=14.0,8.8 \mathrm{~Hz}, 1 \mathrm{H}) .{ }^{13} \mathrm{C}$ NMR $(75$ MHz): $\delta 173.2,162.1\left(\mathrm{~d}, J_{\mathrm{F}-\mathrm{C}}=246.4\right), 137.8,136.8\left(\mathrm{~d}, J_{\mathrm{F}-\mathrm{C}}=2.7\right), 133.9,129.3,128.6\left(\mathrm{~d}, J_{\mathrm{F}-}\right.$ $\left.\mathrm{C}_{\mathrm{C}}=8.2\right), 128.4,115.3\left(\mathrm{~d}, J_{\mathrm{F}-\mathrm{C}}=21.4\right), 70.0,61.9,59.1,52.3,30.9 . \mathrm{FAB}+\mathrm{MS} \mathrm{m} / \mathrm{z} 364.1$ $\left(\mathrm{M}^{+}+\mathrm{H}, 100\right)$. 


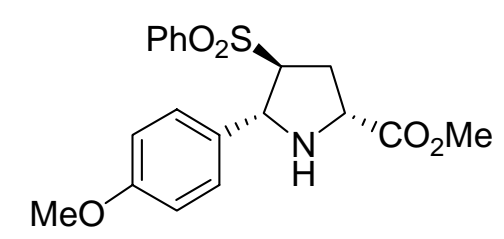

Methyl (2R,4S,5R)-5-(p-methoxyphenyl)-4-phenylsulfonylpyrrolidine-2-carboxylate (5). Yellow oil; yield: $55 \mathrm{mg}(71 \%)$. $[\alpha]_{\mathrm{D}}{ }^{20}=-17\left(\mathrm{c} 0.40, \mathrm{CHCl}_{3}\right), 84 \%$ ee. HPLC: Daicel Chiralpak AS-H, i-PrOH-hexane 40/60, flow rate $0.8 \mathrm{~mL} / \mathrm{min}, t_{\mathrm{R}}: 47.4 \mathrm{~min}(2 R, 4 S, 5 R)-$ isomer and $60.5 \mathrm{~min}(2 S, 4 R, 5 S)$-isomer, $210 \mathrm{~nm} .{ }^{1} \mathrm{H}$ NMR $(300 \mathrm{MHz}): \delta$ 7.85-7.77 (m, 2H), 7.63-7.54 (m, 1H), 7.52-7.40 (m, 2H), $7.13(\mathrm{~d}, J=8.7 \mathrm{~Hz}, 2 \mathrm{H}), 6.74(\mathrm{~d}, J=8.7 \mathrm{~Hz}$, $2 \mathrm{H}), 4.66(\mathrm{~d}, J=6.1 \mathrm{~Hz}, 1 \mathrm{H}), 4.13(\mathrm{t}, J=7.9 \mathrm{~Hz}, 1 \mathrm{H}), 3.84-3.67(\mathrm{~m}, 6 \mathrm{H}), 3.66-3.57(\mathrm{~m}, 1 \mathrm{H})$, 2.70 (ddd, $J=13.9,7.6,4.8 \mathrm{~Hz}, 1 \mathrm{H}), 2.49$ (bs, $1 \mathrm{H}), 2.35(\mathrm{dt}, J=13.8,8.9 \mathrm{~Hz}, 1 \mathrm{H}) .{ }^{13} \mathrm{C}$ NMR (75 MHz): $\delta 173.3,159.1,138.0,133.7,132.9,129.2,128.4,127.9,113.9,69.9,62.3,59.1$, 55.2, 52.3, 31.2. $\mathrm{FAB}+\mathrm{MS} \mathrm{m} / \mathrm{z} 376.1\left(\mathrm{M}^{+}+\mathrm{H}, 100\right)$. HRMS (EI) Calcd for $\mathrm{C}_{19} \mathrm{H}_{22} \mathrm{NO}_{5} \mathrm{~S}$, 376.1222; found, 376.1219 .<smiles>CC(=O)[C@H]1C[C@H](S(=O)(=O)O)[C@H](c2cccc(F)c2)N1</smiles>

Methyl (2R,4S,5R)-5-( $m$-fluorophenyl)-4-phenylsulfonylpyrrolidine-2-carboxylate (6). Orange oil; yield: $60 \mathrm{mg}(83 \%) \cdot[\alpha]_{\mathrm{D}}{ }^{20}=+61\left(\mathrm{c} 0.30, \mathrm{CHCl}_{3}\right) .{ }^{1} \mathrm{H} \mathrm{NMR}(300 \mathrm{MHz}): \delta$ 7.89-7.73 (m, 2H), 7.67-7.59 (m, 1H), 7.55-7.47 (m, 2H), 7.21-7.18 (m, 1H), 7.05-6.94 (m, 2H), 6.92-6.87 (m, 1H), $4.77(\mathrm{~d}, J=5.4 \mathrm{~Hz}, 1 \mathrm{H}), 4.20(\mathrm{t}, J=7.9 \mathrm{~Hz}, 1 \mathrm{H}), 3.76(\mathrm{~s}, 3 \mathrm{H}), 3.65-$ $3.56(\mathrm{~m}, 1 \mathrm{H}), 2.76(\mathrm{ddd}, J=14.0,7.4,4.1 \mathrm{~Hz}, 1 \mathrm{H}), 2.45-2.17(\mathrm{~m}, 2 \mathrm{H}) .{ }^{13} \mathrm{C}$ NMR $(75 \mathrm{MHz})$ : $\delta 173.1,162.8\left(\mathrm{~d}, J_{\mathrm{F}-\mathrm{C}}=246.4 \mathrm{~Hz}\right), 144.0\left(\mathrm{~d}, J_{\mathrm{F}-\mathrm{C}}=7.1 \mathrm{~Hz}\right), 137.8,134.0,130.1\left(\mathrm{~d}, J_{\mathrm{F}-\mathrm{C}}=8.2\right.$ $\mathrm{Hz}), 129.6,128.5,122.4\left(\mathrm{~d}, J_{\mathrm{F}-\mathrm{C}}=2.7 \mathrm{~Hz}\right), 114.7\left(\mathrm{~d}, J_{\mathrm{F}-\mathrm{C}}=21.4 \mathrm{~Hz}\right), 113.8\left(\mathrm{~d}, J_{\mathrm{F}-\mathrm{C}}=22.5 \mathrm{~Hz}\right)$, 70.2, 61.9, 59.2, 52.4, 30.7. FAB+ MS m/z $364.1\left(\mathrm{M}^{+}+\mathrm{H}, 100\right)$. HRMS (EI) Calcd for $\mathrm{C}_{18} \mathrm{H}_{19} \mathrm{FNO}_{4} \mathrm{~S}, 364.1028$; found, 364.1019 .<smiles>COc1cccc([C@@H]2N[C@H](C(C)=O)C[C@H]2S(=O)(=O)c2ccccc2)c1</smiles>

Methyl $(2 R, 4 S, 5 R)-5$-(m-methoxyphenyl)-4-phenylsulfonylpyrrolidine-2-carboxylate (7). Yellow oil; yield: $53 \mathrm{mg}(71 \%)$. [ $\alpha]_{\mathrm{D}}{ }^{20}=-16\left(\mathrm{c} 0.85, \mathrm{CHCl}_{3}\right), 85 \%$ ee. HPLC: Daicel Chiralpak AS-H, i-PrOH-hexane 40/60, flow rate $0.8 \mathrm{~mL} / \mathrm{min}, t_{\mathrm{R}}: 59.4 \mathrm{~min}(2 S, 4 R, 5 S)$ isomer and $67.5 \mathrm{~min}(2 R, 4 S, 5 R)$-isomer, $210 \mathrm{~nm} .{ }^{1} \mathrm{H} \mathrm{NMR}(300 \mathrm{MHz}): \delta 7.87-7.75(\mathrm{~m}$, 2H), 7.65-7.55 (m, 1H), 7.54-7.41 (m, 2H), 7.16-7.08 (m, 1H), 6.82-6.70 (m, 3H), $4.72(\mathrm{~d}$, $J=5.3 \mathrm{~Hz}, 1 \mathrm{H}), 4.19(\mathrm{t}, J=7.9 \mathrm{~Hz}, 1 \mathrm{H}), 3.78-3.71(\mathrm{~m}, 6 \mathrm{H}), 3.69-3.59(\mathrm{~m}, 1 \mathrm{H}), 2.69$ (ddd, $J=13.9,7.4,4.8 \mathrm{~Hz}, 1 \mathrm{H}), 2.49$ (bs, $1 \mathrm{H}), 2.45-2.27$ (m, 2H). ${ }^{13} \mathrm{C}$ NMR $(75 \mathrm{MHz}): \delta 173.3$, 159.7, 142.9, 137.9, 133.8, 129.6, 129.2, 128.5, 118.8, 113.4, 112.3, 70.3, 62.6, 59.4, 55.1, 52.3, 30.9. FAB+ MS m/z $376.0\left(\mathrm{M}^{+}+\mathrm{H}, 100\right)$. HRMS (EI) Calcd for $\mathrm{C}_{19} \mathrm{H}_{22} \mathrm{NO}_{5} \mathrm{~S}$, 376.1232; found, 376.1219 . 
<smiles>COC(=O)[C@@H]1C[C@H](SO)[C@H](c2cccc(C)c2)N1</smiles>

Methyl $(2 R, 4 S, 5 R)$-4-phenylsulfonyl-5-( $m$-tolyl)pyrrolidine-2-carboxylate (8). White solid; yield: $53 \mathrm{mg}(74 \%)$; mp 93-94 ${ }^{\circ} \mathrm{C} .[\alpha]_{\mathrm{D}}{ }^{20}=-19\left(\mathrm{c} 0.27, \mathrm{CHCl}_{3}\right) .{ }^{1} \mathrm{H}$ NMR $(300$ MHz): $\delta$ 7.87-7.75 (m, 2H), 7.63-7.54 (m, 1H), 7.51-7.42 (m, 2H), 7.15-7.05 (m, 1H), 7.04-6.95 (m, 2H), 6.94-6.87 (m, 1H), $4.65(\mathrm{~d}, J=5.8 \mathrm{~Hz}, 1 \mathrm{H}), 4.17(\mathrm{t}, J=7.8 \mathrm{~Hz}, 1 \mathrm{H}), 3.76$ (s, 3H), 3.71-3.61 (m, 1H), 2.80-2.64 (m, 1H), 2.62-2.30 (m, 2H), $2.24(\mathrm{~s}, 3 \mathrm{H}) .{ }^{13} \mathrm{C}$ NMR (75 MHz): $\delta$ 173.2, 140.6, 138.1, 138.0, 133.8, 129.1, 128.6, 128.5, 127.5, 123.8, 70.0, 62.9, 59.3, 52.3, 31.1, 21.3. FAB+ MS m/z $360.0\left(\mathrm{M}^{+}+\mathrm{H}, 100\right)$. HRMS (EI) Calcd for $\mathrm{C}_{19} \mathrm{H}_{22} \mathrm{NO}_{4} \mathrm{~S}, 360.1263$; found, 360.1269 .<smiles>CC(=O)[C@@H]1C[C@H](S(=O)(=O)Oc2ccccc2)[C@H](c2ccc3ccccc3c2)N1</smiles>

Methyl (2R,4S,5R)-5-(2-naphthyl)-4-phenylsulfonylpyrrolidine-2-carboxylate (9). White solid; yield: $56 \mathrm{mg}(71 \%)$; $\mathrm{mp} 147-148^{\circ} \mathrm{C} .[\alpha]_{\mathrm{D}}{ }^{20}=-46\left(\mathrm{c} 0.50, \mathrm{CHCl}_{3}\right), 65 \%$ ee. HPLC: Daicel Chiralpak AS-H, i-PrOH-hexane 40/60, flow rate $0.8 \mathrm{~mL} / \mathrm{min}, t_{\mathrm{R}}: 46.3 \mathrm{~min}$ $(2 S, 4 R, 5 S)$-isomer and $50.4 \mathrm{~min}(2 R, 4 S, 5 R)$-isomer, $210 \mathrm{~nm} .{ }^{1} \mathrm{H}$ NMR $(300 \mathrm{MHz})$ : $\delta 7.85-$ $7.65(\mathrm{~m}, 5 \mathrm{H}), 7.61(\mathrm{bs}, 1 \mathrm{H}), 7.54-7.32(\mathrm{~m}, 6 \mathrm{H}), 4.90(\mathrm{~d}, J=5.5 \mathrm{~Hz}, 1 \mathrm{H}), 4.25(\mathrm{t}, J=7.9 \mathrm{~Hz}$, $1 \mathrm{H}), 3.82-3.69(\mathrm{~m}, 4 \mathrm{H}), 2.83-2.53(\mathrm{~m}, 2 \mathrm{H}), 2.43(\mathrm{dt}, J=14.0,8.9 \mathrm{~Hz}, 1 \mathrm{H}) .{ }^{13} \mathrm{C}$ NMR $(75$ MHz): $\delta 173.2,138.3,137.9,133.8,133.0,132.9,129.2,128.5,128.5,127.9,127.5,126.2$, 126.1, 126.0, 124.2, 70.0, 62.8, 59.3, 52.3, 30.9. FAB+ MS m/z $396.2\left(\mathrm{M}^{+}+\mathrm{H}, 100\right)$. HRMS (EI) Calcd for $\mathrm{C}_{22} \mathrm{H}_{22} \mathrm{NO}_{4} \mathrm{~S}, 396.1272$; found, 396.1269 .<smiles>CC(=O)[C@@H]1C[C@H](S(=O)(=O)O)[C@H](c2ccccc2C)N1</smiles>

Methyl $(2 R, 4 S, 5 R)$-4-phenylsulfonyl-5-(o-tolyl)pyrrolidine-2-carboxylate (10). White solid; yield: $66 \mathrm{mg}(92 \%)$; $\mathrm{mp} 112-113{ }^{\circ} \mathrm{C} .[\alpha]_{\mathrm{D}}{ }^{20}=-16\left(\mathrm{c} 0.50, \mathrm{CHCl}_{3}\right), 41 \%$ ee. HPLC: Daicel Chiralpak AS-H, i-PrOH-hexane 40/60, flow rate $0.8 \mathrm{~mL} / \mathrm{min}, t_{\mathrm{R}}: 26.8 \mathrm{~min}$ $(2 S, 4 R, 5 S)$-isomer and $30.1 \mathrm{~min}(2 R, 4 S, 5 R)$-isomer, $210 \mathrm{~nm} .{ }^{1} \mathrm{H}$ NMR $(300 \mathrm{MHz}): \delta 7.84-$ 7.74 (m, 2H), 7.63-7.53 (m, 1H), 7.51-7.41 (m, 2H), 7.15-7.06 (m, 1H), 7.05-6.96 (m, 2H), $6.92(\mathrm{bs}, 1 \mathrm{H}), 4.65(\mathrm{~d}, J=5.9 \mathrm{~Hz}, 1 \mathrm{H}), 4.17(\mathrm{t}, J=7.9 \mathrm{~Hz}, 1 \mathrm{H}), 3.77(\mathrm{~s}, 3 \mathrm{H}), 3.72-3.70(\mathrm{~m}$, $1 \mathrm{H}), 2.72$ (ddd, $J=14.0,7.1,4.4 \mathrm{~Hz}, 1 \mathrm{H}), 2.60-2.30(\mathrm{~m}, 2 \mathrm{H}), 2.24(\mathrm{~s}, 3 \mathrm{H}) .{ }^{13} \mathrm{C}$ NMR $(75$ MHz): $\delta 173.2,140.6,138.1,138.0,133.8,129.1,129.2,128.6,128.5,127.5,123.8,70.0$, 62.9, 59.3, 52.3, 31.1, 21.3. FAB+ MS m/z $360.0\left(\mathrm{M}^{+}+\mathrm{H}, 100\right)$. HRMS (EI) Calcd for $\mathrm{C}_{19} \mathrm{H}_{22} \mathrm{NO}_{4} \mathrm{~S}, 360.1264$; found, 360.1269 . 


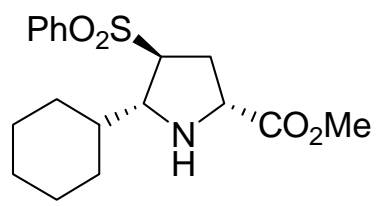

Methyl (2R,4S,5R)-5-(cyclohexyl)-4-phenylsulfonylpyrrolidine-2-carboxylate (11). Colourless oil; yield: $35 \mathrm{mg}(50 \%)$. $[\alpha]_{\mathrm{D}}{ }^{20}=+3\left(\mathrm{c} 0.31, \mathrm{CHCl}_{3}\right), 69 \%$ ee. HPLC: Daicel Chiralpak AS-H, i-PrOH-hexane 40/60, flow rate $0.8 \mathrm{~mL} / \mathrm{min}, t_{\mathrm{R}}: 22.7 \mathrm{~min}(2 R, 4 S, 5 R)-$ isomer and $25.3 \mathrm{~min}(2 S, 4 R, 5 S)$-isomer, $210 \mathrm{~nm} .{ }^{1} \mathrm{H}$ NMR $(300 \mathrm{MHz}): \delta$ 7.95-7.85 (m, $2 \mathrm{H}), 7.72-7.54(\mathrm{~m}, 3 \mathrm{H}), 3.99(\mathrm{dd}, J=9.6,7.1 \mathrm{~Hz}, 1 \mathrm{H}), 3.71(\mathrm{~s}, 3 \mathrm{H}), 3.57-3.39(\mathrm{~m}, 2 \mathrm{H}), 2.50$ (ddd, $J=14.1,6.9,2.5 \mathrm{~Hz}, 1 \mathrm{H}), 2.20-1.96(\mathrm{~m}, 2 \mathrm{H}), 1.77-1.54(\mathrm{~m}, 5 \mathrm{H}), 1.32-0.81(\mathrm{~m}, 6 \mathrm{H})$. ${ }^{13} \mathrm{C}$ NMR (75 MHz): $\delta 173.4,138.2,133.9,129.4,128.7,66.0,63.9,59.3,52.2,42.1,31.7$, 30.3, 28.2, 26.2, 26.1, 25.9. FAB+ MS m/z $352.0\left(\mathrm{M}^{+}+\mathrm{H}, 100\right)$. HRMS (EI) Calcd for $\mathrm{C}_{18} \mathrm{H}_{26} \mathrm{NO}_{4} \mathrm{~S}, 352.1584$; found, 352.1583 .

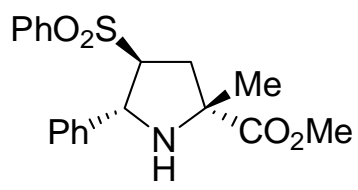

Methyl (2R,4S,5R)-2-methyl-5-phenyl-4-phenylsulfonylpyrrolidine-2-carboxylate (12). White solid; yield: $32 \mathrm{mg}(45 \%)$; $\mathrm{mp} 82-83{ }^{\circ} \mathrm{C} .[\alpha]_{\mathrm{D}}{ }^{20}=-5\left(\mathrm{c} 0.30, \mathrm{CHCl}_{3}\right), 80 \%$ ee. HPLC: Daicel Chiralpak AS-H, i-PrOH-hexane 40/60, flow rate $0.8 \mathrm{~mL} / \mathrm{min}, t_{\mathrm{R}}: 16.6 \mathrm{~min}$ $(2 S, 4 R, 5 S)$-isomer and $19.9 \mathrm{~min}(2 R, 4 S, 5 R)$-isomer, $210 \mathrm{~nm} .{ }^{1} \mathrm{H}$ NMR $(300 \mathrm{MHz}): \delta 7.74-$ $7.65(\mathrm{~m}, 2 \mathrm{H}), 7.53-7.44(\mathrm{~m}, 1 \mathrm{H}), 7.39-7.32(\mathrm{~m}, 2 \mathrm{H}), 7.15(\mathrm{bs}, 5 \mathrm{H}), 4.66(\mathrm{~d}, J=8.3 \mathrm{~Hz}, 1 \mathrm{H})$, 3.87 (c, $J=8.6, \mathrm{~Hz}, 1 \mathrm{H}), 3.71(\mathrm{~s}, 3 \mathrm{H}), 2.79$ (dd, $J=13.7,8.5 \mathrm{~Hz}, 1 \mathrm{H}), 2.61$ (bs, 1H), 2.36 $(\mathrm{dd}, J=13.6,9.6 \mathrm{~Hz}, 1 \mathrm{H}), 1.56(\mathrm{~s}, 3 \mathrm{H}) .{ }^{13} \mathrm{C}$ NMR $(75 \mathrm{MHz}): \delta 176.2,139.8,138.4,133.6$, 129.0, 128.5, 128.2, 127.8, 127.1, 69.8, 65.0, 63.3, 52.6, 38.5, 26.0. IE MS m/z 360.1 $\left(\mathrm{M}^{+}+\mathrm{H}, 100\right)$. HRMS (EI) Calcd for $\mathrm{C}_{19} \mathrm{H}_{22} \mathrm{NO}_{4} \mathrm{~S}, 360.1268$; found, 346.1269 .

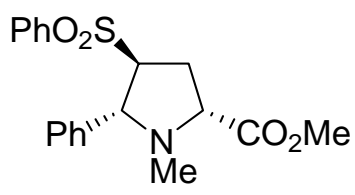

Typical procedure for $N$-methylation of cycloadducts. Methyl $(2 R, 4 S, 5 R)-1$-methyl-5phenyl-4-phenylsulfonylpyrrolidine-2-carboxylate (13). To a solution of 3a (500 mg, 1.4 $\mathrm{mmol})$ and $\mathrm{K}_{2} \mathrm{CO}_{3}(796 \mathrm{mg}, 5.8 \mathrm{mmol})$ in DMF $(14 \mathrm{~mL})$ was added at $\mathrm{rt} \mathrm{MeI}(130 \mu \mathrm{L}, 2.1$ $\mathrm{mmol})$. The mixture was stirred for $4 \mathrm{~h}$ and then $\mathrm{Et}_{2} \mathrm{O}(20 \mathrm{~mL})$ was added. The mixture was washed with $\mathrm{H}_{2} \mathrm{O}(3 \times 10 \mathrm{~mL})$ and the aqueous phase was extracted with $\mathrm{Et}_{2} \mathrm{O}(20 \mathrm{~mL})$. The combined organic phase was dried $\left(\mathrm{MgSO}_{4}\right)$ and concentrated. The residue was purified by flash cromathography ( $n$-hexane-EtOAc 2:1) to afford 13 as white solid; yield: $477 \mathrm{mg}$ $(92 \%) ; \mathrm{mp} 112-113{ }^{\circ} \mathrm{C} .[\alpha]_{\mathrm{D}}{ }^{20}=-34\left(\mathrm{c} 0.50, \mathrm{CHCl}_{3}\right), 99 \%$ ee. HPLC: Daicel Chiralpak AS-H, i-PrOH-hexane 40/60, flow rate $0.8 \mathrm{~mL} / \mathrm{min}, t_{\mathrm{R}}: 12.2 \mathrm{~min}(2 R, 4 S, 5 R)$-isomer and $15.2 \mathrm{~min}(2 S, 4 R, 5 S)$-isomer, $210 \mathrm{~nm} .{ }^{1} \mathrm{H}$ NMR $(300 \mathrm{MHz}): \delta 7.77-7.69(\mathrm{~m}, 2 \mathrm{H}), 7.58-7.50$ $(\mathrm{m}, 1 \mathrm{H}), 7.46-7.37(\mathrm{~m}, 2 \mathrm{H}), 7.21-7.09(\mathrm{~m}, 5 \mathrm{H}), 3.88(\mathrm{dd}, J=6.4 \mathrm{~Hz}, 1 \mathrm{H}), 3.72(\mathrm{~s}, 3 \mathrm{H}), 3.60$ $(\mathrm{m}, 1 \mathrm{H}), 3.50(\mathrm{dd}, J=9.7,7.2 \mathrm{~Hz}, 1 \mathrm{H}), 2.61(\mathrm{ddd}, J=13.9,7.1,3.6 \mathrm{~Hz}, 1 \mathrm{H}), 2.38(\mathrm{dt}, J=$ 
13.9, $9.9 \mathrm{~Hz}, 1 \mathrm{H}), 2.22(\mathrm{~s}, 3 \mathrm{H}) .{ }^{13} \mathrm{C}$ NMR (75 MHz): $\delta$ 172.1, 140.0, 137.8, 133.7, 129.1, 128.4, 128.3, 123.6, 127.4, 70.1, 68.9, 66.0, 51.9, 38.8, 29.8.

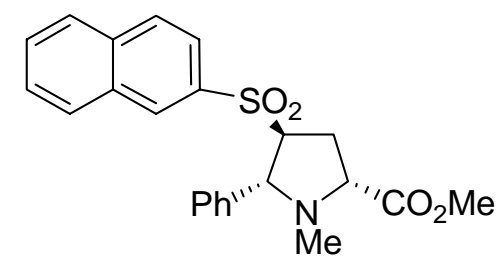

Methyl $\quad(2 R, 4 S, 5 R)$-1-methyl-4-(2-naphthyl)sulfonyl-5-phenylpyrrolidine-2-carboxylate. White solid; yield: $510 \mathrm{mg}(89 \%)$; $\mathrm{mp} 130-131{ }^{\circ} \mathrm{C}$. $[\alpha]_{\mathrm{D}}{ }^{20}=-9$ (c $\left.0.30, \mathrm{CHCl}_{3}\right), 75 \%$ ee. HPLC: Daicel Chiralpak AS-H, i-PrOH-hexane 40/60, flow rate $0.8 \mathrm{~mL} / \mathrm{min}, t_{\mathrm{R}}: 19.2$ $\min (2 R, 4 S, 5 R)$-isomer and $21.9 \min (2 S, 4 R, 5 S)$-isomer, $210 \mathrm{~nm} .{ }^{1} \mathrm{H}$ NMR $(300 \mathrm{MHz}): \delta$ 8.39 (bs, $1 \mathrm{H}), 7.97-7.80(\mathrm{~m}, 3 \mathrm{H}), 7.71-7.57(\mathrm{~m}, 3 \mathrm{H}), 7.23-7.14(\mathrm{~m}, 2 \mathrm{H}), 7.10-7.00(\mathrm{~m}, 3 \mathrm{H})$, $3.95(\mathrm{~d}, J=6.7 \mathrm{~Hz}, 1 \mathrm{H}), 3.81-3.71(\mathrm{~m}, 4 \mathrm{H}), 3.56(\mathrm{dd}, J=9.5,7.4 \mathrm{~Hz}, 1 \mathrm{H}), 2.70$ (ddd, $J=$ 13.9, 7.4, 4.0 Hz, 1H ), 2.42 (dt, $J=13.8,9.9 \mathrm{~Hz}, 1 \mathrm{H}), 2.25$ (s, 3H). ${ }^{13} \mathrm{C} \mathrm{NMR}(75 \mathrm{MHz}): \delta$ $172.3,139.7,135.1,134.5,131.8,130.4,129.4,129.3,129.2,128.1,127.8,127.7,127.6$, $127.5,122.6,70.3,68.9,66.1,52.0,38.8,29.8$.<smiles>CC(=O)[C@@H]1C[C@H](SO)[C@H](c2cccc(F)c2)N1C</smiles>

Methyl (2R,4S,5R)-5-( $m$-fluorophenyl)-1-methyl-4-phenylsulfonylpyrrolidine-2-carboxylate. White solid; yield: $449 \mathrm{mg}(85 \%)$; $\mathrm{mp} 96-97^{\circ} \mathrm{C}$. $[\alpha]_{\mathrm{D}}{ }^{20}=-30\left(\mathrm{c} 0.53, \mathrm{CHCl}_{3}\right), 85 \%$ ee. HPLC: Daicel Chiralpak AS-H, i-PrOH-hexane 40/60, flow rate $0.8 \mathrm{~mL} / \mathrm{min}, t_{\mathrm{R}}: 12.9$ $\min (2 R, 4 S, 5 R)$-isomer and $19.7 \mathrm{~min}(2 S, 4 R, 5 S)$-isomer, $210 \mathrm{~nm} .{ }^{1} \mathrm{H}$ NMR $(300 \mathrm{MHz}): \delta$ 7.85-7.71 (m, 2H), 7.65-7.57 (m, 1H), 7.53-7.44 (m, 2H), 7.21-7.11 (m, 1H), 7.02-6.83 (m, $3 \mathrm{H}), 3.94(\mathrm{~d}, J=6.1 \mathrm{~Hz}, 1 \mathrm{H}), 3.76(\mathrm{~s}, 3 \mathrm{H}), 3.62-3.47(\mathrm{~m}, 2 \mathrm{H}), 2.61$ (ddd, $J=13.9,7.1,3.5$ $\mathrm{Hz}, 1 \mathrm{H}), 2.37$ (dt, $J=13.9,9.9 \mathrm{~Hz}, 1 \mathrm{H}), 2.27$ (s, 3H). ${ }^{13} \mathrm{C}$ NMR $(75 \mathrm{MHz}): \delta 172.2,162.8$ $\left(\mathrm{d}, J_{\mathrm{F}-\mathrm{C}}=246.4\right), 143.1\left(\mathrm{~d}, J_{\mathrm{F}-\mathrm{C}}=7.1 \mathrm{~Hz}\right), 137.8,133.9,130.0\left(\mathrm{~d}, J_{\mathrm{F}-\mathrm{C}}=8.2 \mathrm{~Hz}\right), 129.3,128.6$, $123.2\left(\mathrm{~d}, J_{\mathrm{F}-\mathrm{C}}=2.7 \mathrm{~Hz}\right), 114.7\left(\mathrm{~d}, J_{\mathrm{F}-\mathrm{C}}=20.9 \mathrm{~Hz}\right), 114.3\left(\mathrm{~d}, J_{\mathrm{F}-\mathrm{C}}=22.5 \mathrm{~Hz}\right), 69.6,69.2,66.1$, $52.1,39.0,30.0$

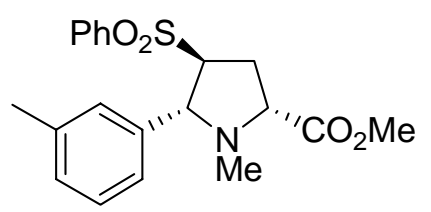

Methyl (2R,4S,5R)-1-methyl-4-phenylsulfonyl-5-( $m$-tolyl)pyrrolidine-2-carboxylate. White solid; yield: $459 \mathrm{mg}(88 \%)$; $\mathrm{mp} 89-90{ }^{\circ} \mathrm{C} .[\alpha]_{\mathrm{D}}{ }^{20}=-39\left(\mathrm{c} 0.54, \mathrm{CHCl}_{3}\right), 79 \%$ ee. HPLC: Daicel Chiralpak AS-H, i-PrOH-hexane 40/60, flow rate $0.8 \mathrm{~mL} / \mathrm{min}, t_{\mathrm{R}}: 14.4 \mathrm{~min}$ $(2 R, 4 S, 5 R)$-isomer and $18.9 \mathrm{~min}(2 S, 4 R, 5 S)$-isomer, $210 \mathrm{~nm} .{ }^{1} \mathrm{H}$ NMR $(300 \mathrm{MHz}): \delta 7.80-$ $7.73(\mathrm{~m}, 2 \mathrm{H}), 7.61-7.53(\mathrm{~m}, 1 \mathrm{H}), 7.49-7.40(\mathrm{~m}, 2 \mathrm{H}), 7.11-7.94(\mathrm{~m}, 3 \mathrm{H}), 6.86(\mathrm{bs}, 1 \mathrm{H}), 3.81$ $(\mathrm{d}, J=6.4 \mathrm{~Hz}, 1 \mathrm{H}), 3.76(\mathrm{~s}, 3 \mathrm{H}), 3.67-3.58(\mathrm{~m}, 1 \mathrm{H}), 3.50(\mathrm{dd}, J=9.7,7.2 \mathrm{~Hz}, 1 \mathrm{H}), 2.66$ (ddd, $J=13.8,7.1,3.6 \mathrm{~Hz}, 1 \mathrm{H}), 2.41$ (dt, $J=13.9,9.9 \mathrm{~Hz} 1 \mathrm{H}), 2.23$ (s, 3H), 2.21 (s, 3H). 
${ }^{13} \mathrm{C}$ NMR (75 MHz): $\delta 172.3,139.9,138.0,137.9,133.7,129.1,128.6,128.5,128.3,128.2$, $124.6,70.5,69.0,66.2,52.1,38.9,29.8,21.2$.

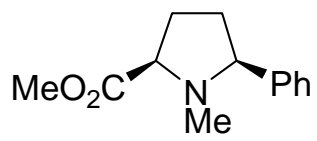

Typical procedure for desulfonilation: Methyl (2R, 5S)-1-methyl-5-phenylpyrrolidine2-carboxylate (14) and methyl $(R, E)$-2-methylamino-5-phenylpentanoate (15). A mixture of a $99 \%$ ee sample of sulfone $3 \mathbf{a}(128 \mathrm{mg}, 0.36 \mathrm{mmol}), 5 \% \mathrm{Na}(\mathrm{Hg})(570 \mathrm{mg})$ and $\mathrm{Na}_{2} \mathrm{HPO}_{4}(186 \mathrm{mg}, 1.3 \mathrm{mmol})$ in a 2:1 mixture of MeOH/THF $(6 \mathrm{~mL})$ was stirred for $5 \mathrm{~h}$ at rt. Then it was poured into $\mathrm{CH}_{2} \mathrm{Cl}_{2}(20 \mathrm{~mL})$, filtered and washed with water $(2 \times 10 \mathrm{~mL})$. The aqueous phase was extracted with $\mathrm{CH}_{2} \mathrm{Cl}_{2}(3 \times 10 \mathrm{~mL})$ and the combined organic phase was dried $\left(\mathrm{MgSO}_{4}\right)$ and concentrated. The residue was purified by flash chromathography ( $n$-hexane-EtOAc 4:1 $\rightarrow$ EtOAc) $\mathbf{1 4}$ followed by 15, both as a colourless oil:

14: Yield: $48 \mathrm{mg}(61 \%)$. $[\alpha]_{\mathrm{D}}{ }^{20}=-55\left(\mathrm{c} 0.45, \mathrm{CHCl}_{3}\right), 99 \%$ ee. HPLC: Daicel Chiralpak AS-H, i-PrOH-hexane 5/95, flow rate $0.3 \mathrm{~mL} / \mathrm{min}, t_{\mathrm{R}}$ : $14.8 \mathrm{~min}(2 R, 5 S)$-isomer and 16.2 $\min (2 S, 5 R)$-isomer, $210 \mathrm{~nm} .{ }^{1} \mathrm{H}$ NMR $(300 \mathrm{MHz}): \delta$ 7.37-7.12 $(\mathrm{m}, 5 \mathrm{H}), 3.70 \quad(\mathrm{~s}, 3 \mathrm{H})$, 3.32-3.25 (m, 1H), 3.16-3.09 (m, 1H), $2.16(\mathrm{~s}, 3 \mathrm{H}), 2.12-1.95(\mathrm{~m}, 3 \mathrm{H}), 1.82-1.70(\mathrm{~m}, 1 \mathrm{H})$. ${ }^{13} \mathrm{C}$ NMR (75 MHz): $\delta$ 174.4, 142.7, 128.3, 127.4, 127.2, 71.8, 68.0, 51.8, 39.8, 34.5, 27.8.

15: Yield: $23 \mathrm{mg}(29 \%) \cdot[\alpha]_{\mathrm{D}}{ }^{20}=-8\left(\mathrm{c} 0.25, \mathrm{CHCl}_{3}\right), 99 \%$ ee. HPLC: Daicel Chiralpak AS-H, i-PrOH-hexane 40/60, flow rate $0.8 \mathrm{~mL} / \mathrm{min}, t_{\mathrm{R}}: 4.9 \mathrm{~min}(R)$-isomer and $5.2 \mathrm{~min}(S)$ isomer, $210 \mathrm{~nm} .{ }^{1} \mathrm{H}$ NMR $(300 \mathrm{MHz}): \delta 7.30-7.10(\mathrm{~m}, 5 \mathrm{H}), 6.39(\mathrm{~d}, J=15.7 \mathrm{~Hz}, 1 \mathrm{H})$, 6.06(dt, $J=15.7,7.4 \mathrm{~Hz}, 1 \mathrm{H}) 3.66(\mathrm{~s}, 3 \mathrm{H}), 3.26(\mathrm{t}, J=6.3 \mathrm{~Hz}, 1 \mathrm{H}), 2.54-2.47(\mathrm{~m}, 1 \mathrm{H}), 2.33$ (s, 3H), 1.56 (bs, $1 \mathrm{H}) .{ }^{13} \mathrm{C}$ NMR $(75 \mathrm{MHz}): \delta 174.9,137.1,133.2,128.5,127.3,126.2$, $124.9,63.0,51.7,36.8,34.7$. 

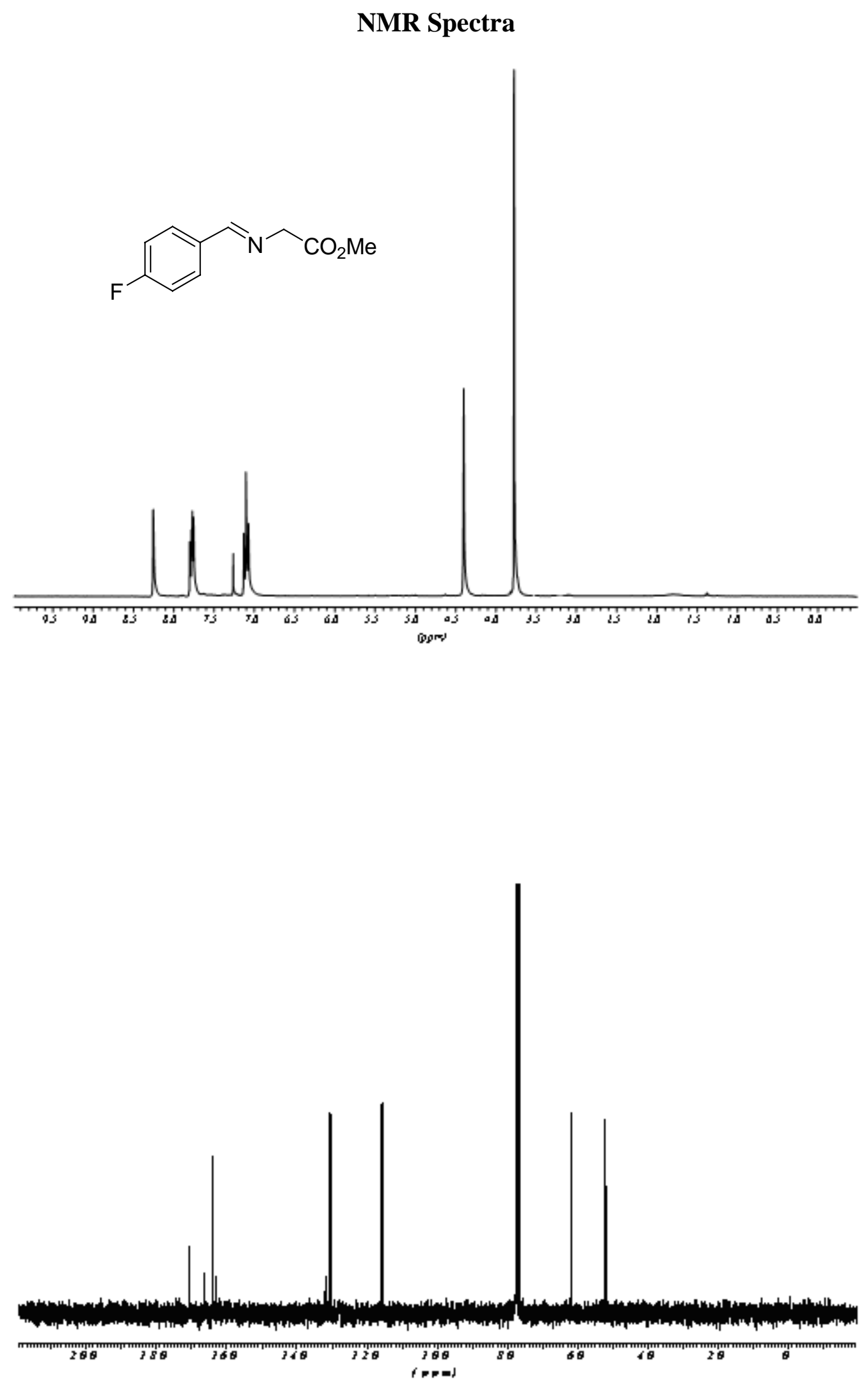


$$
\text { Lلب }
$$



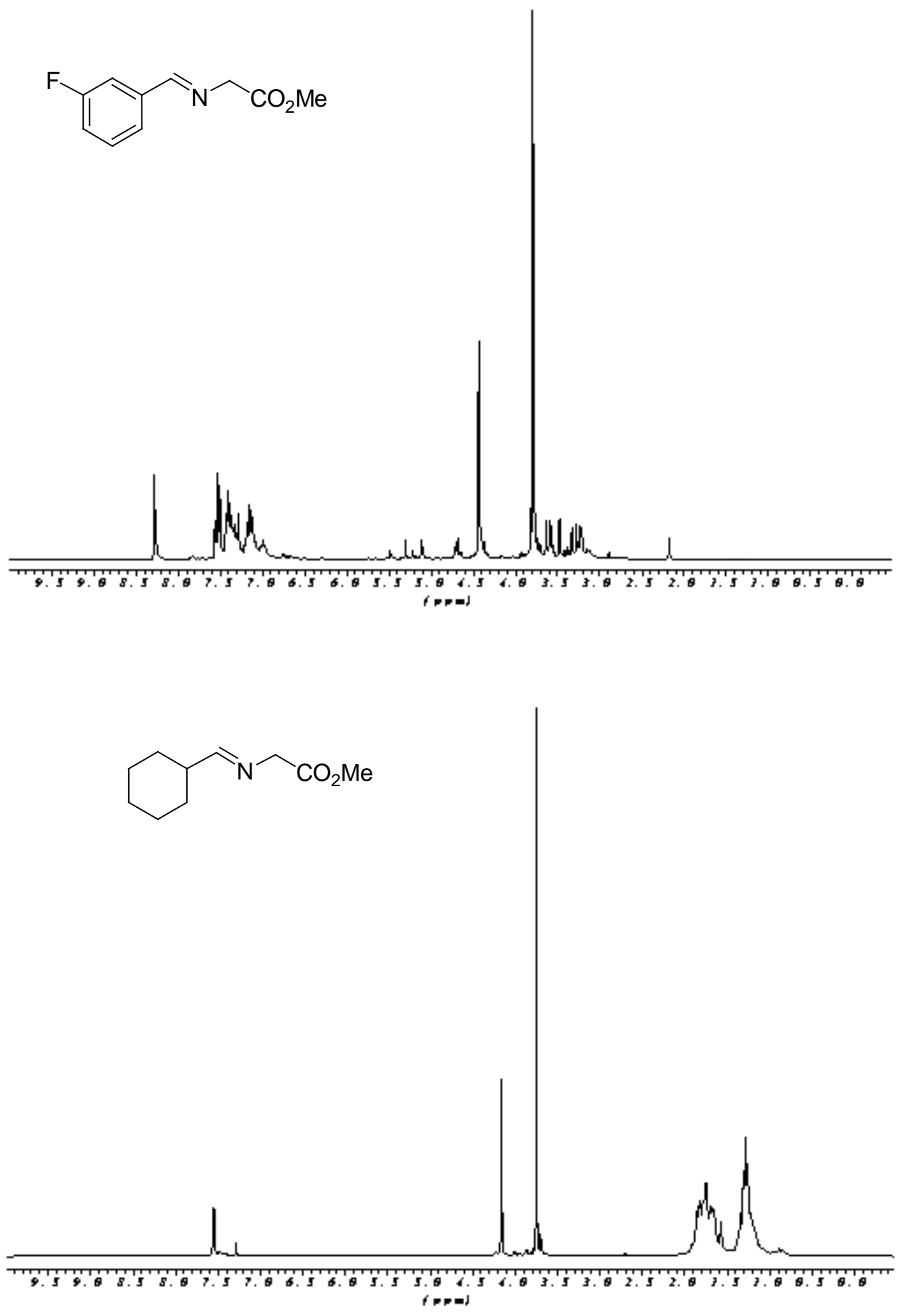

S 14 

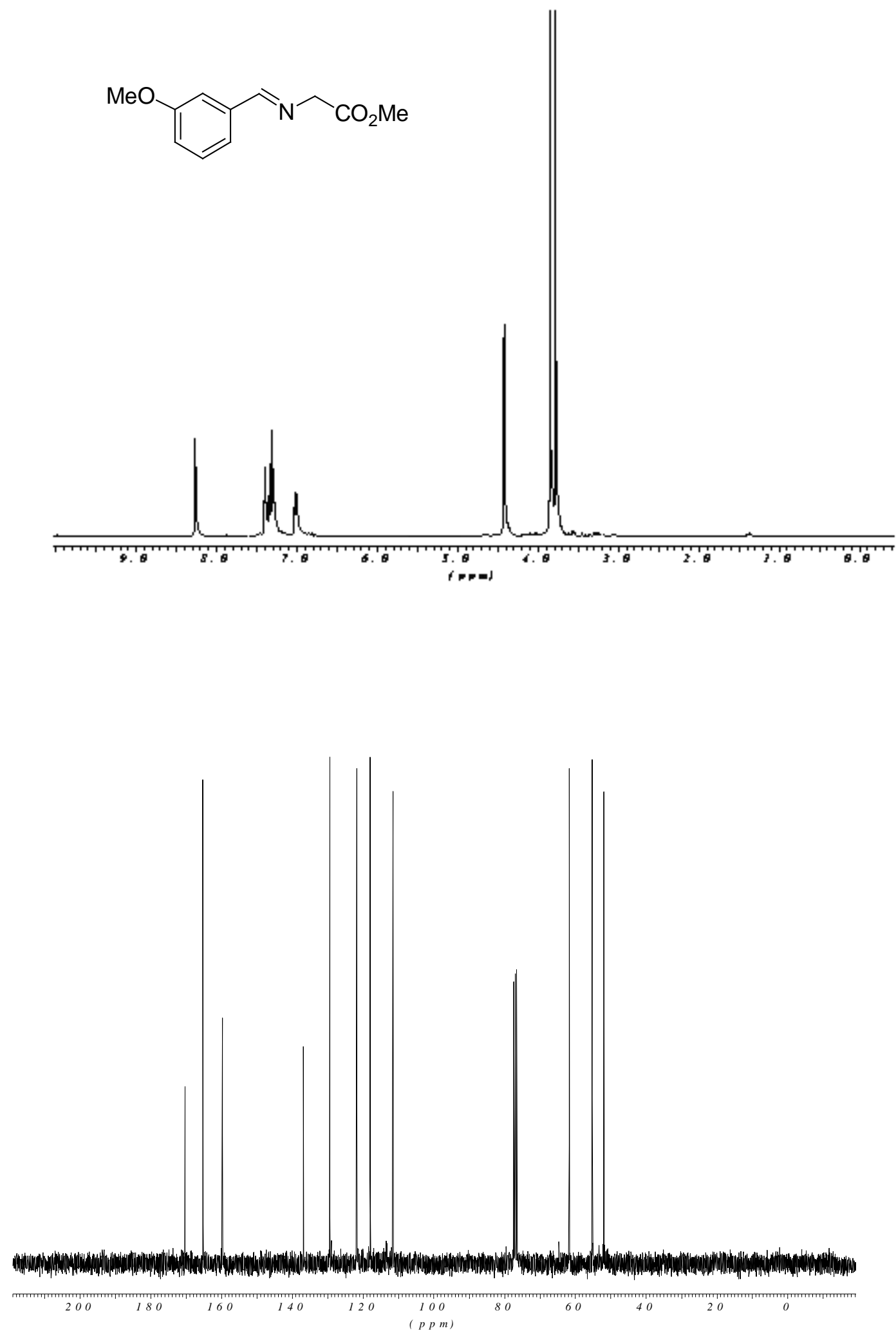

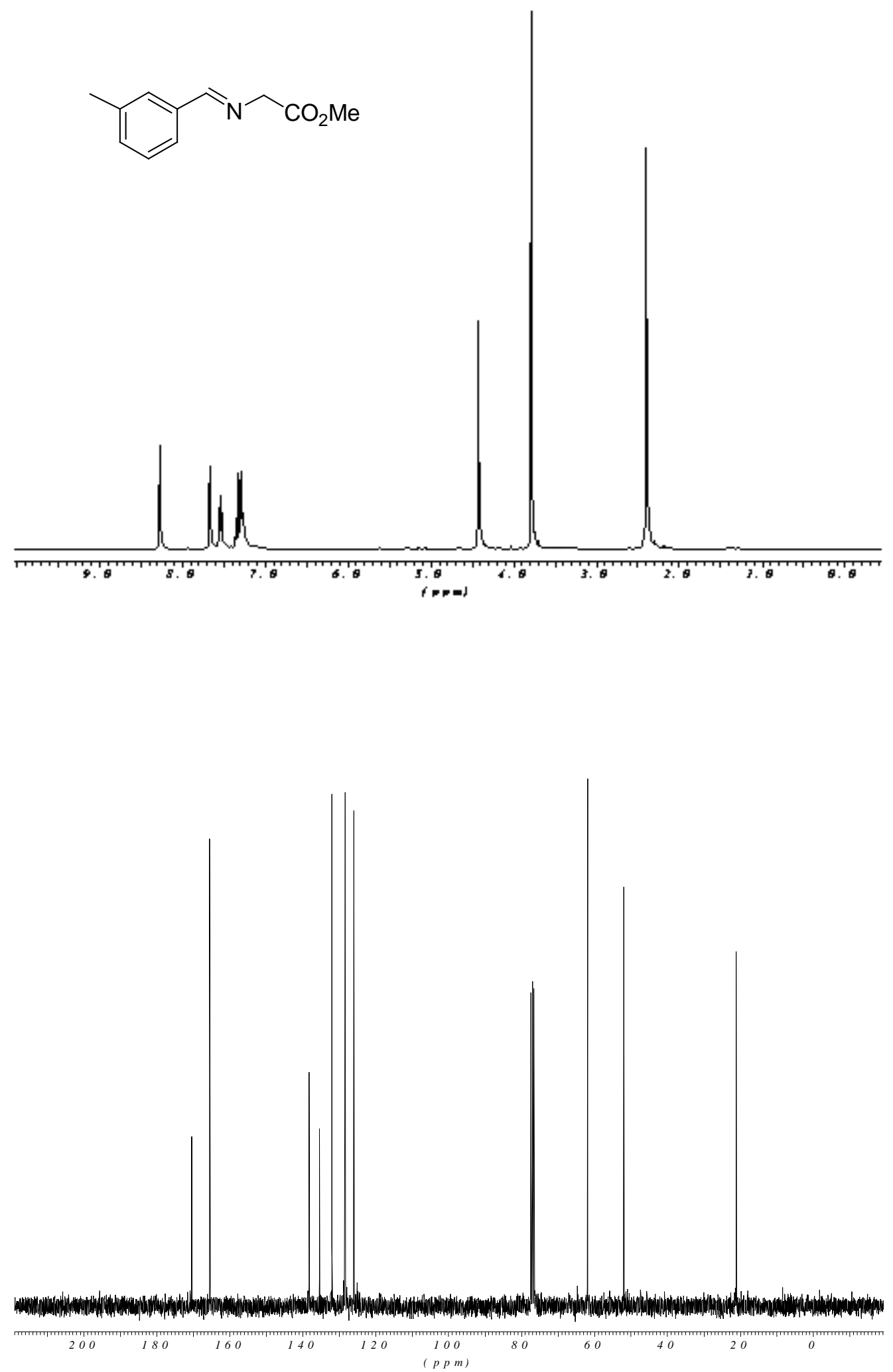

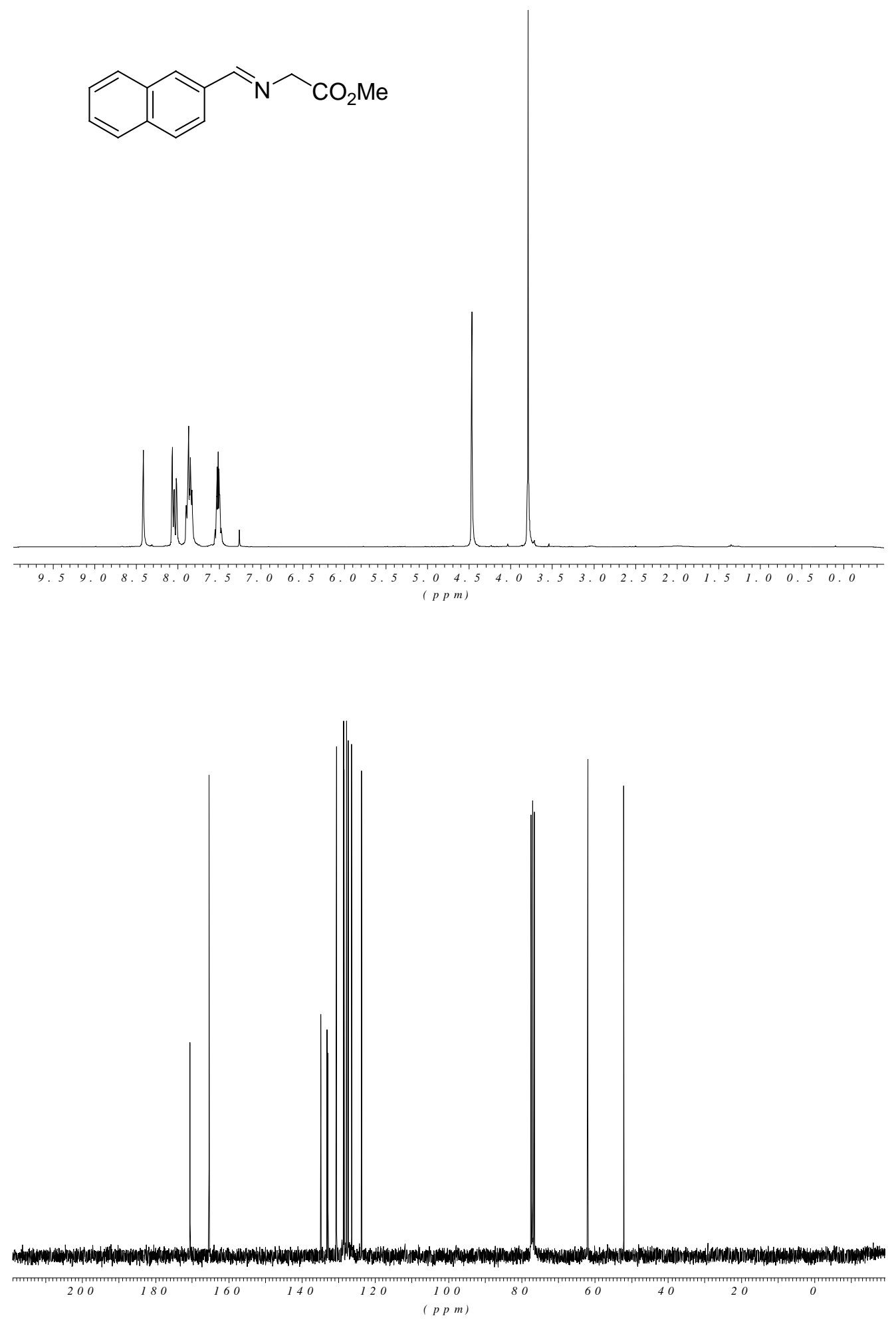

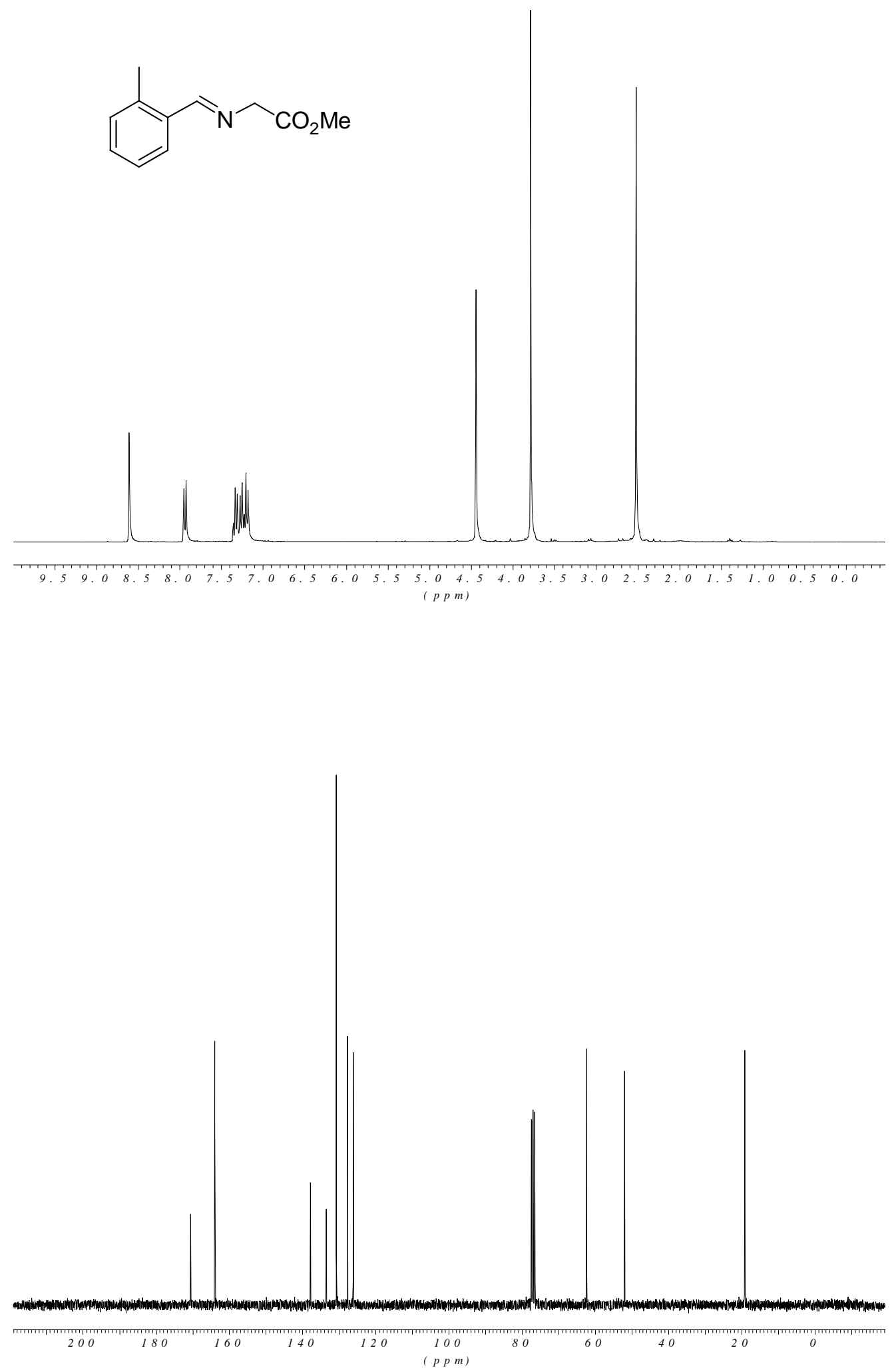

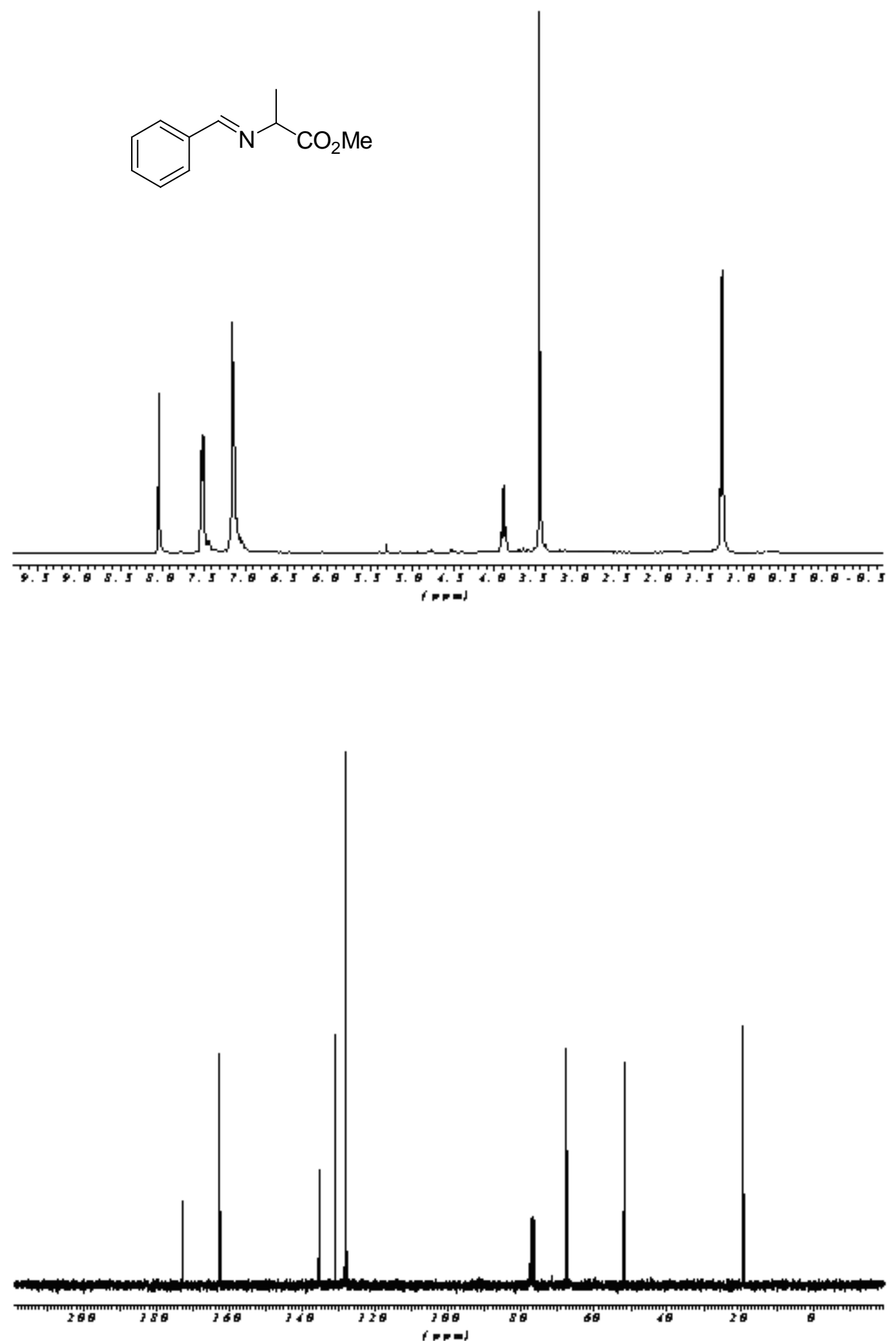


$$
\frac{\|^{\infty}}{+}
$$




$$
\frac{\mathbb{H}^{\circ}}{4}
$$



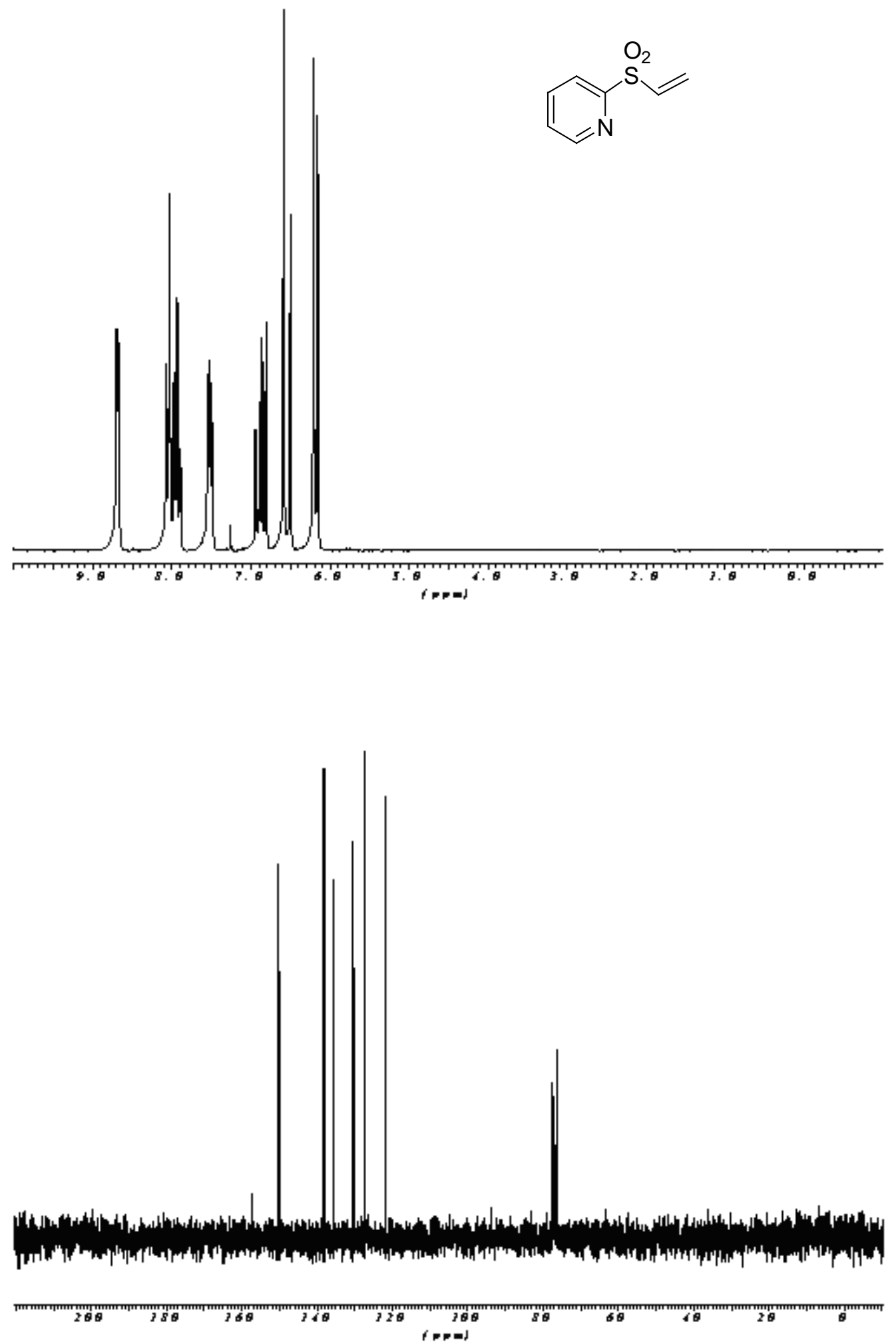

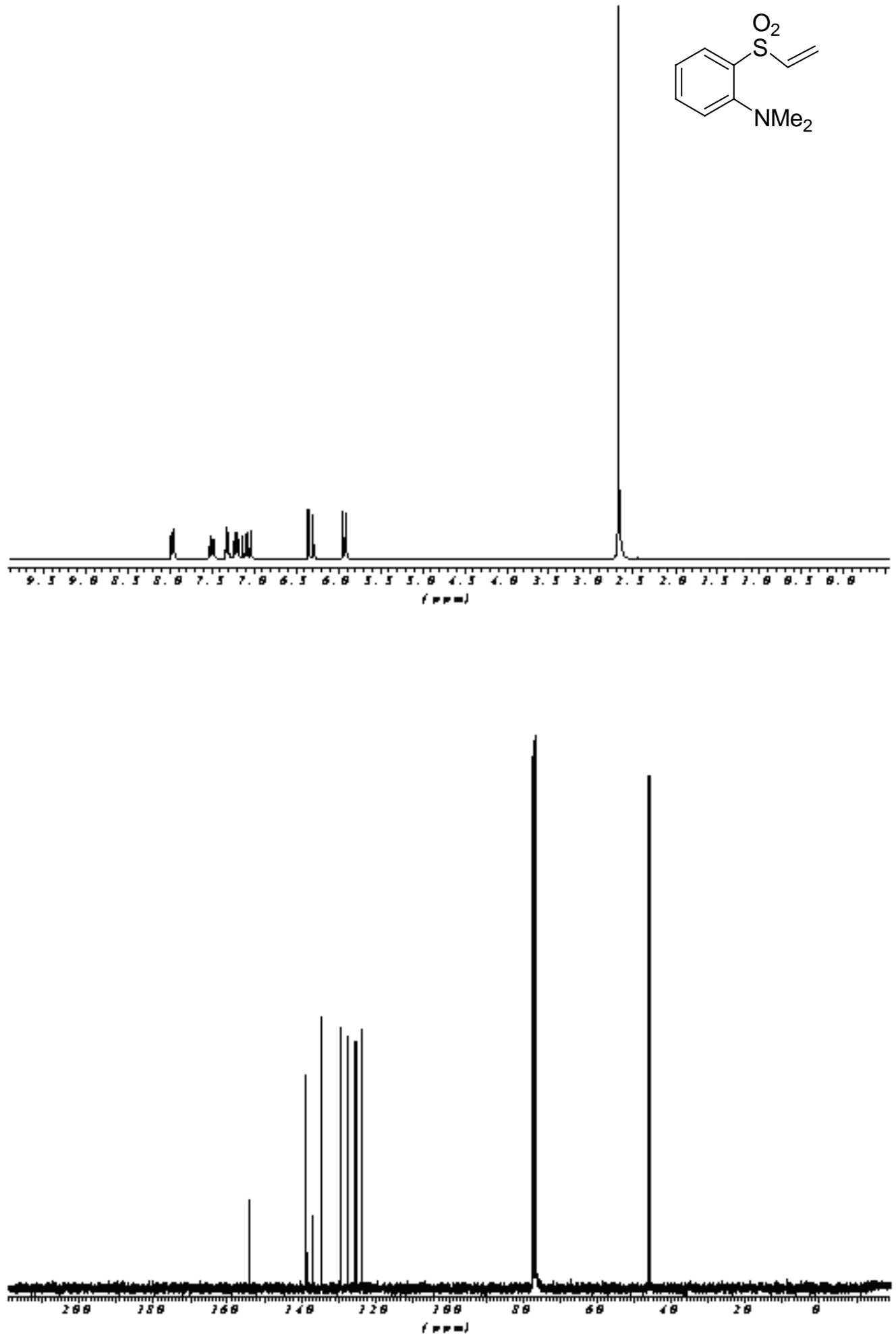

S 23 

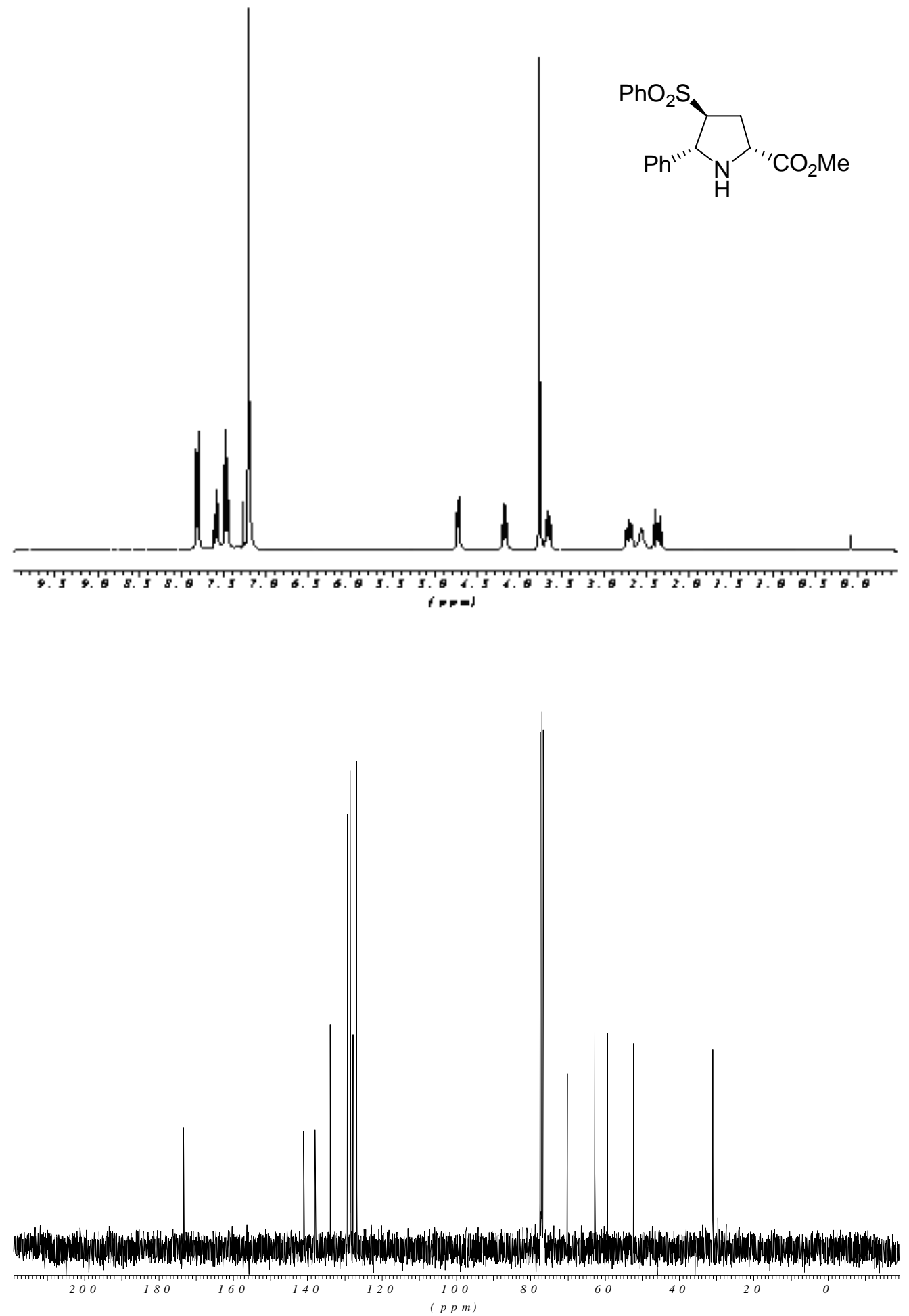

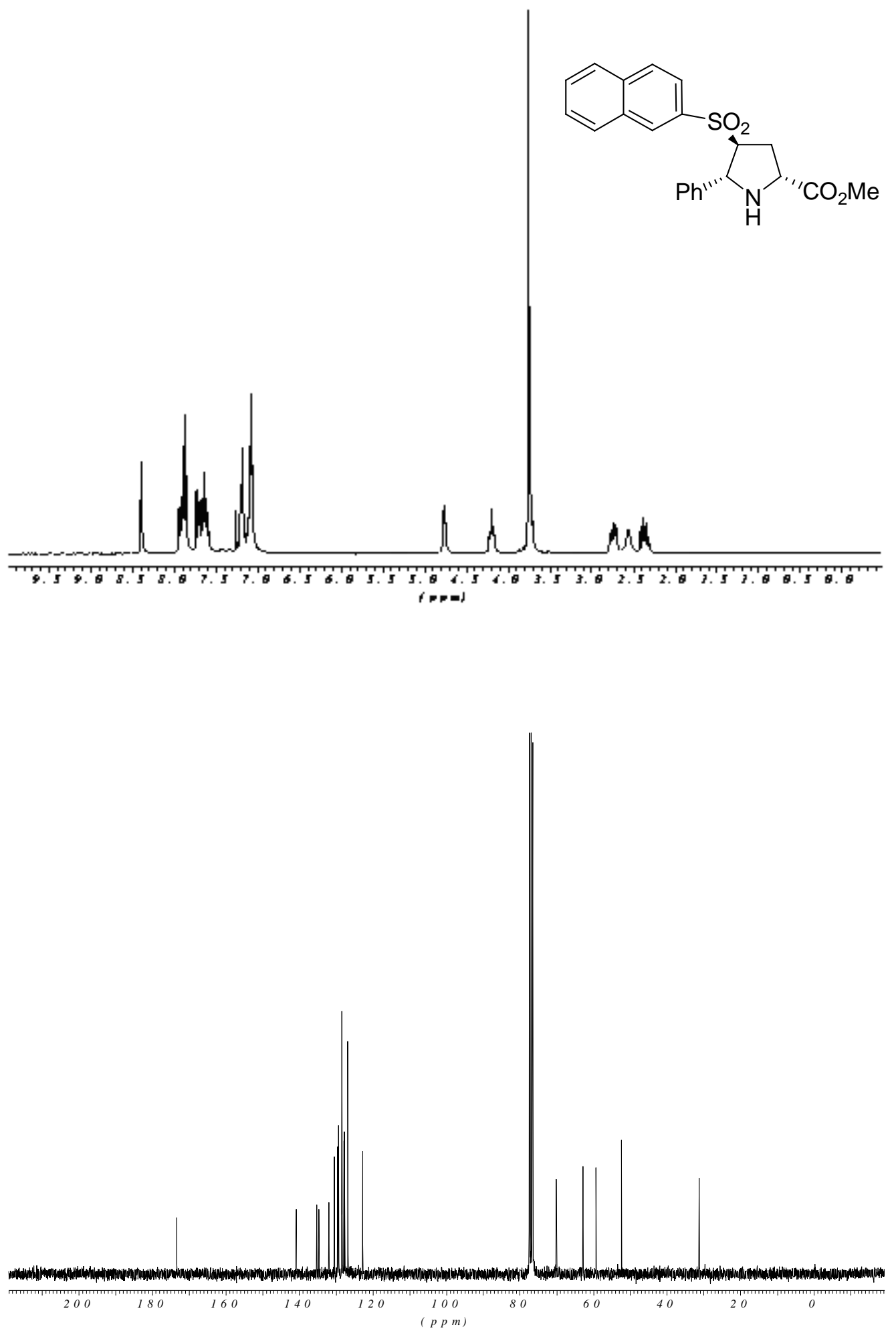

S 25 

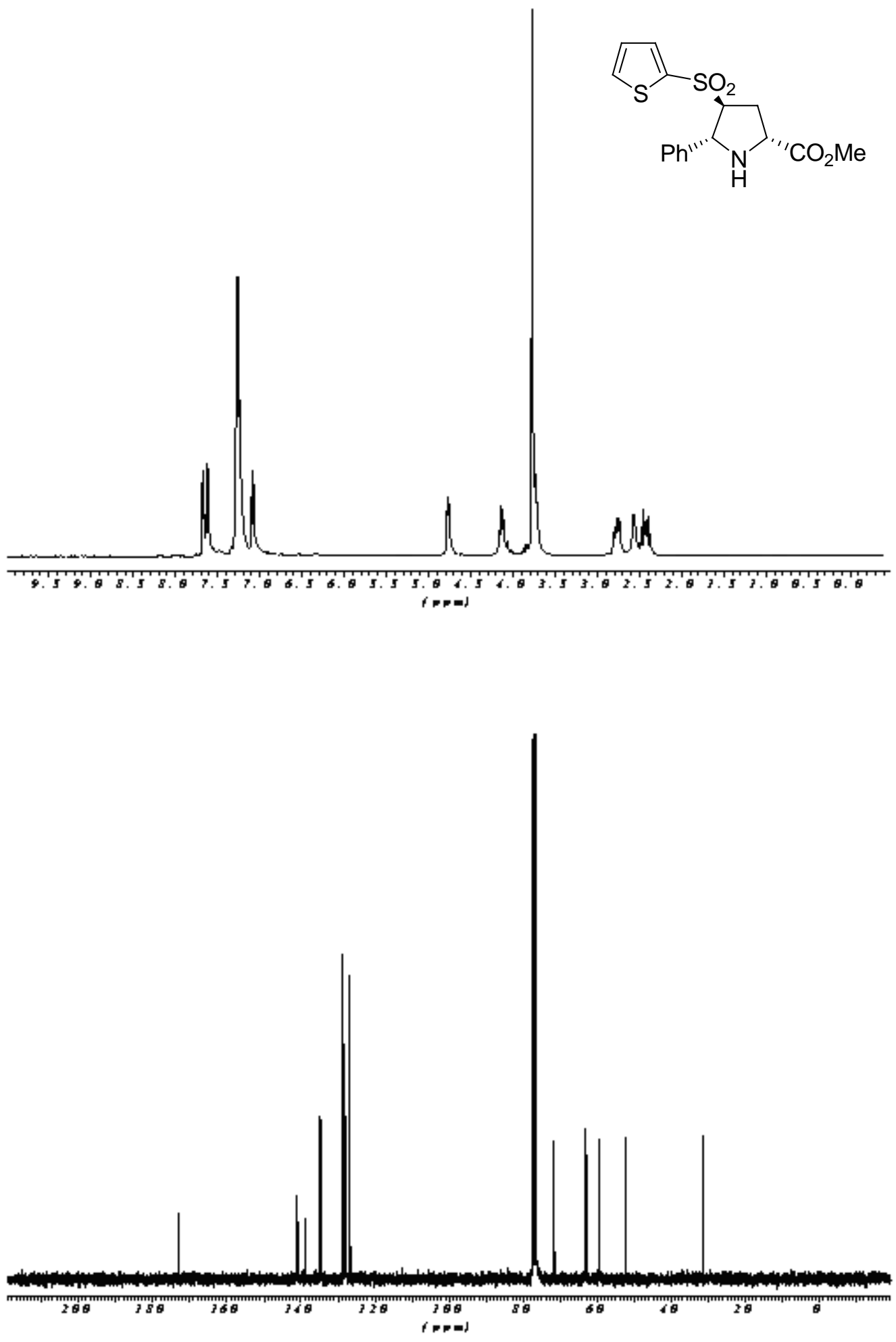

S 26 

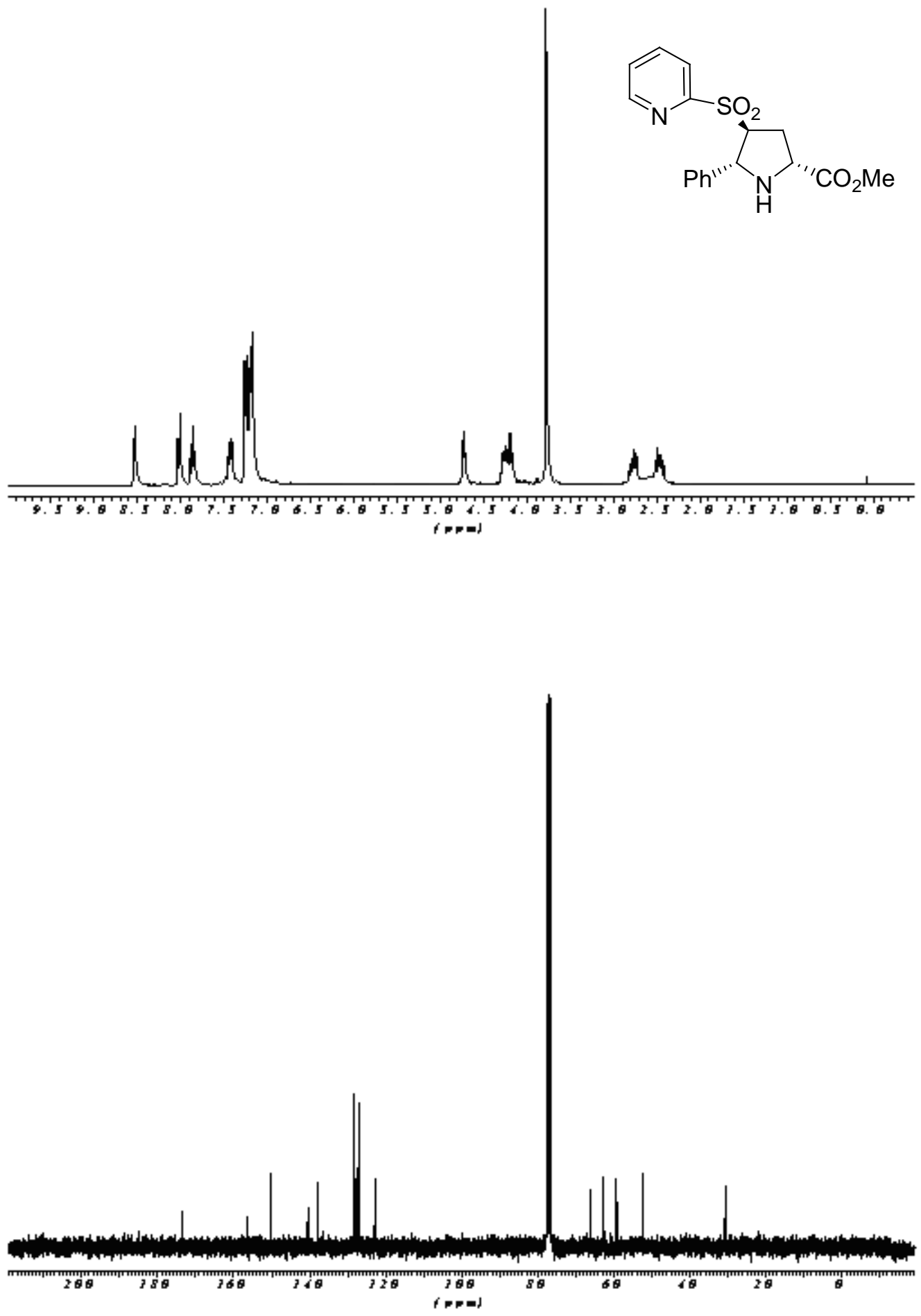

S 27 

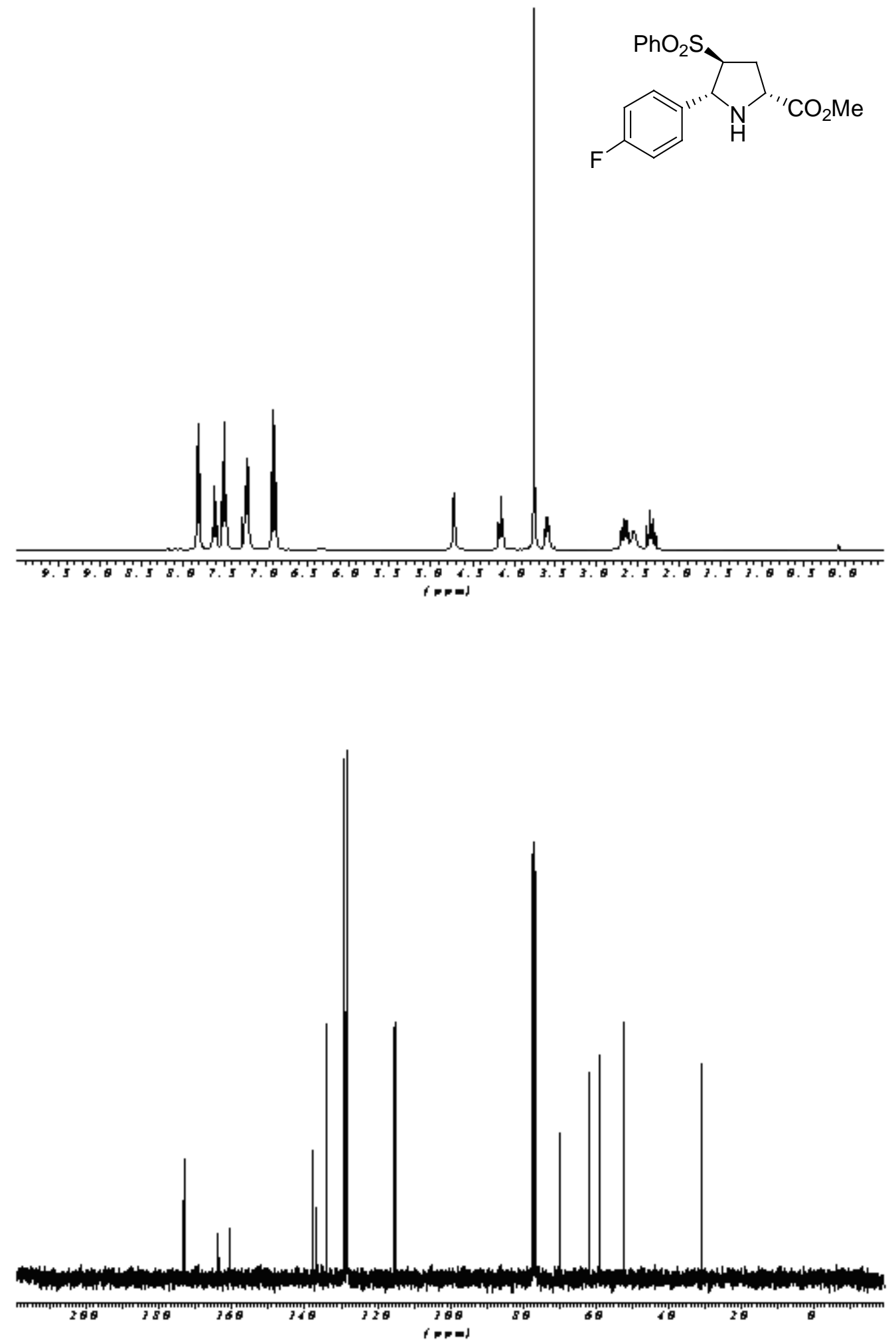

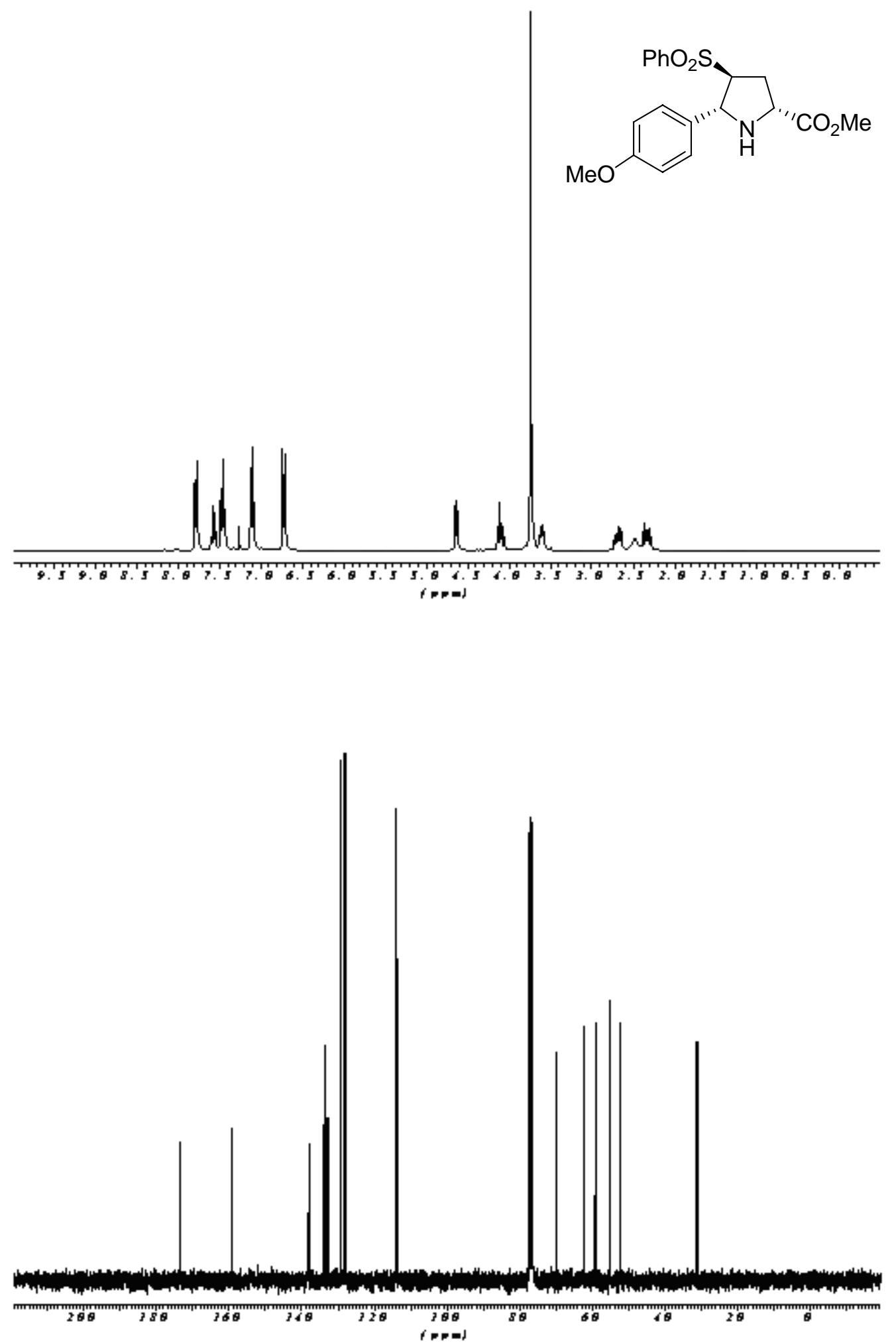

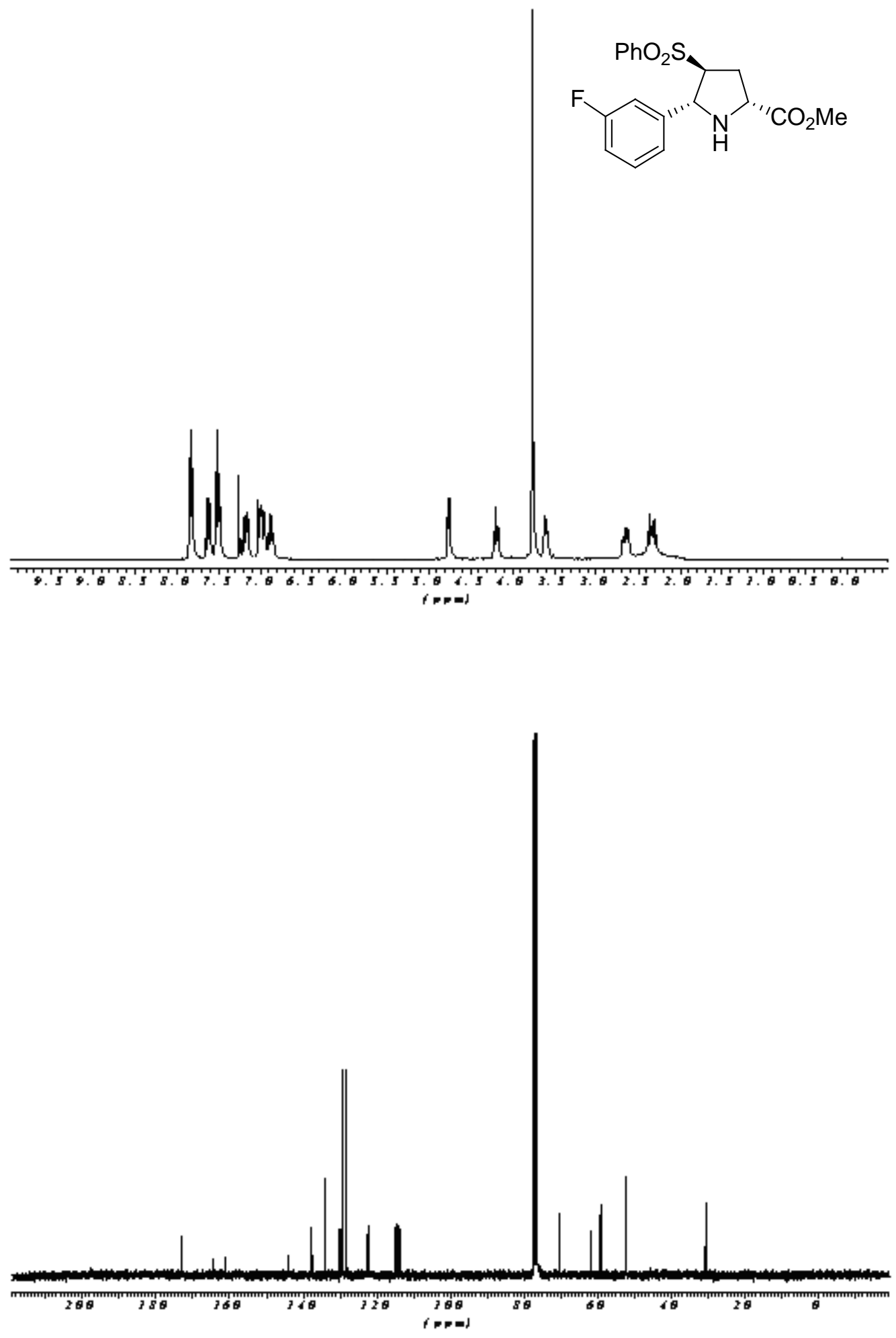

S 30 

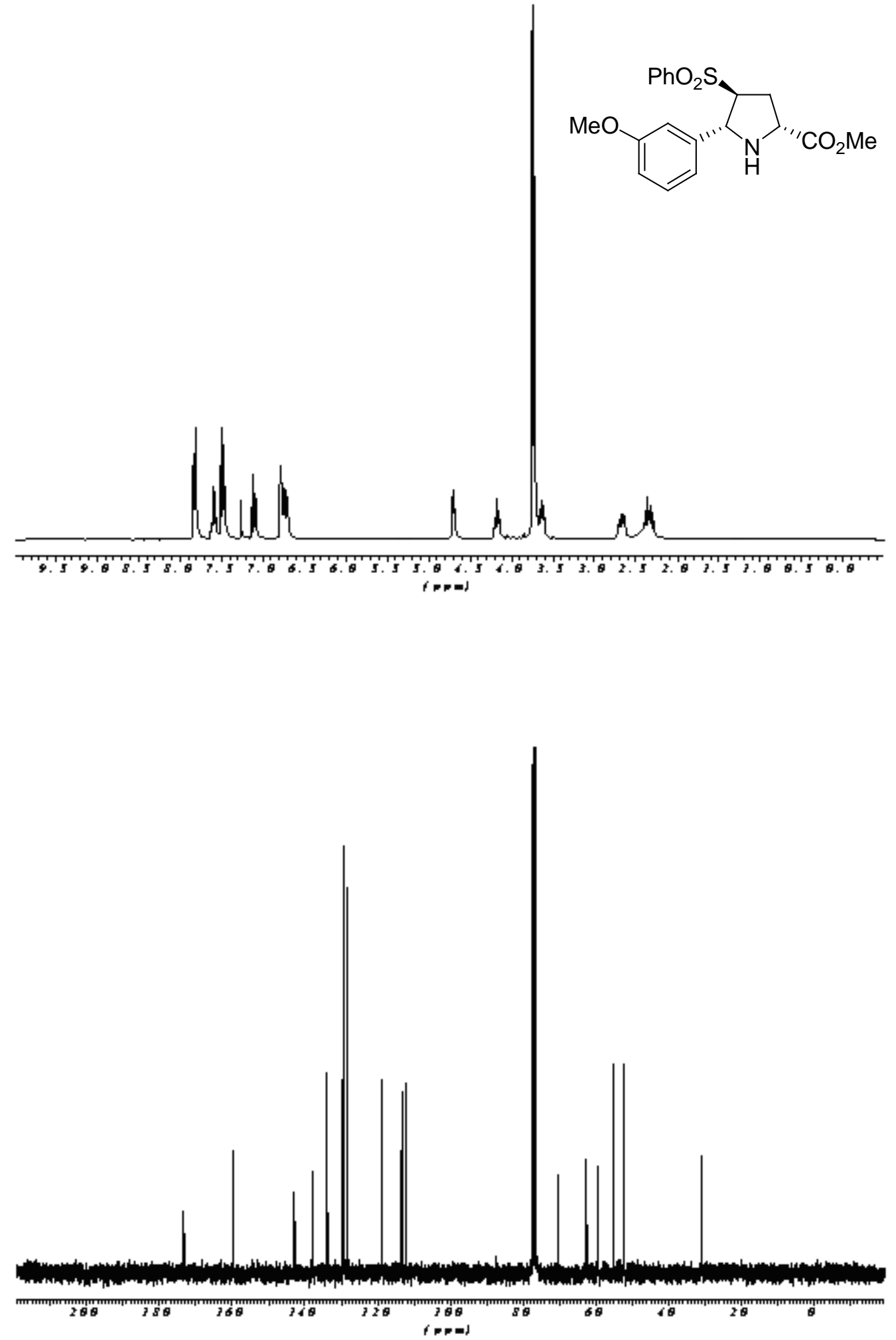

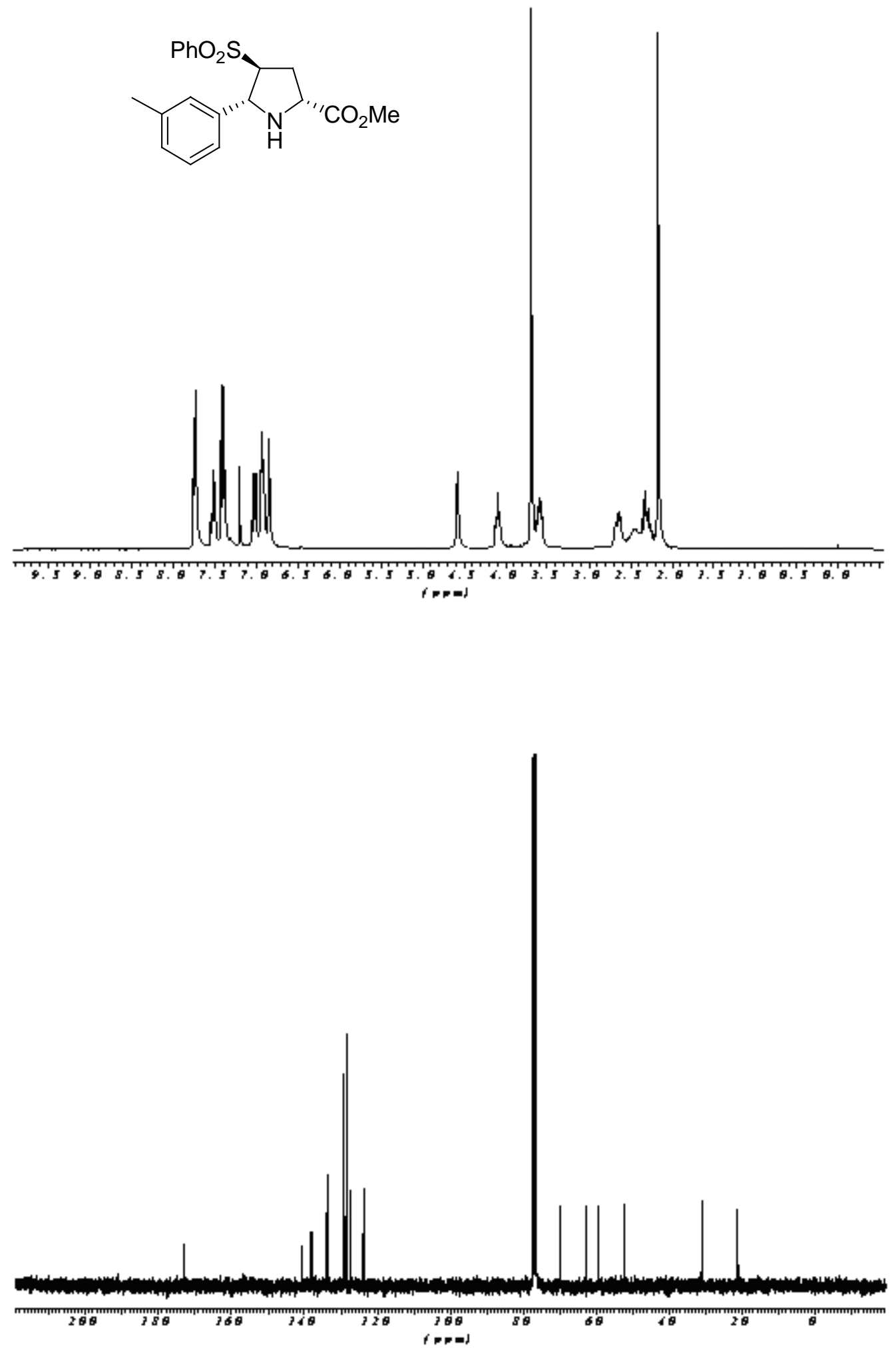

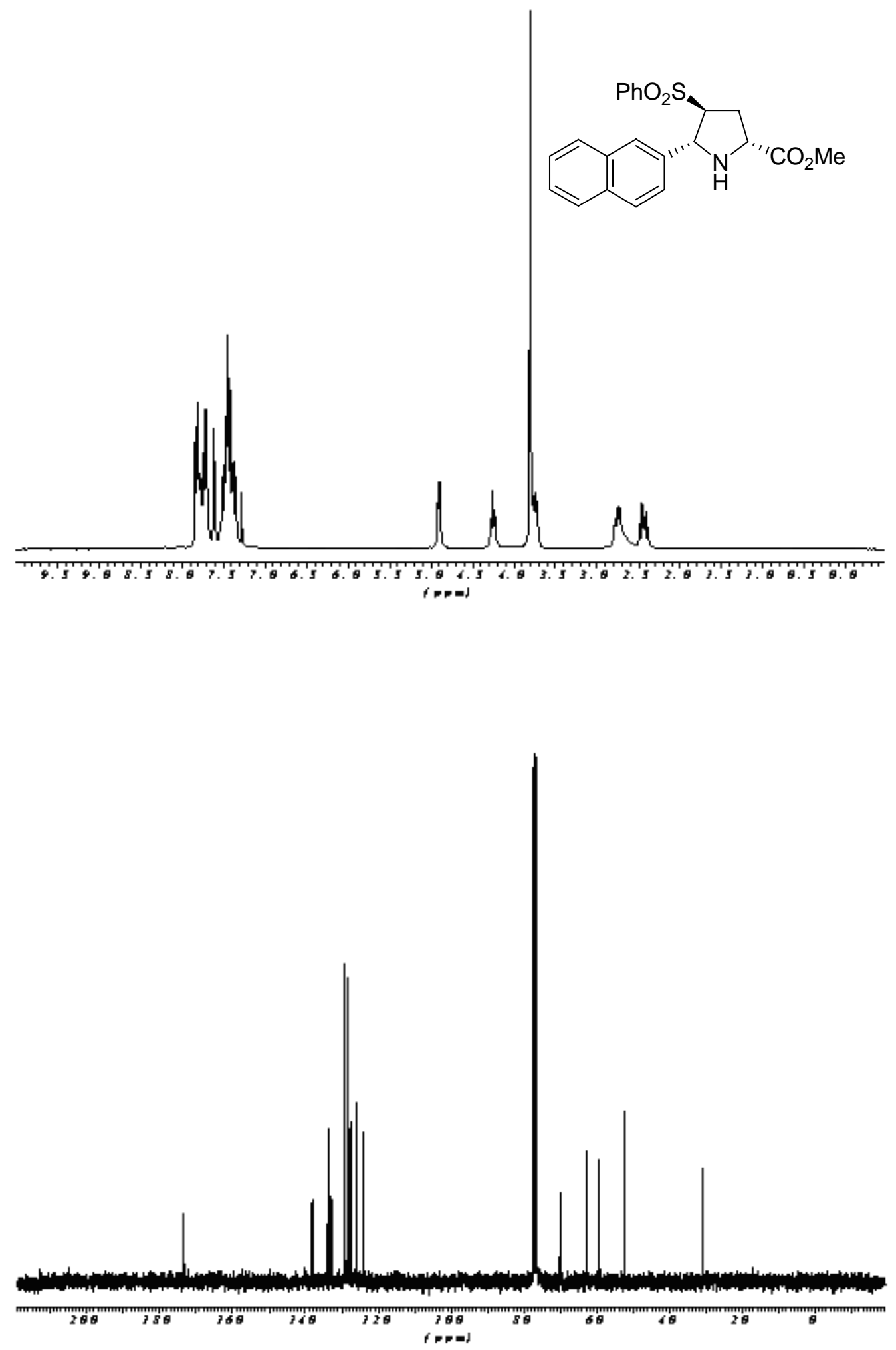

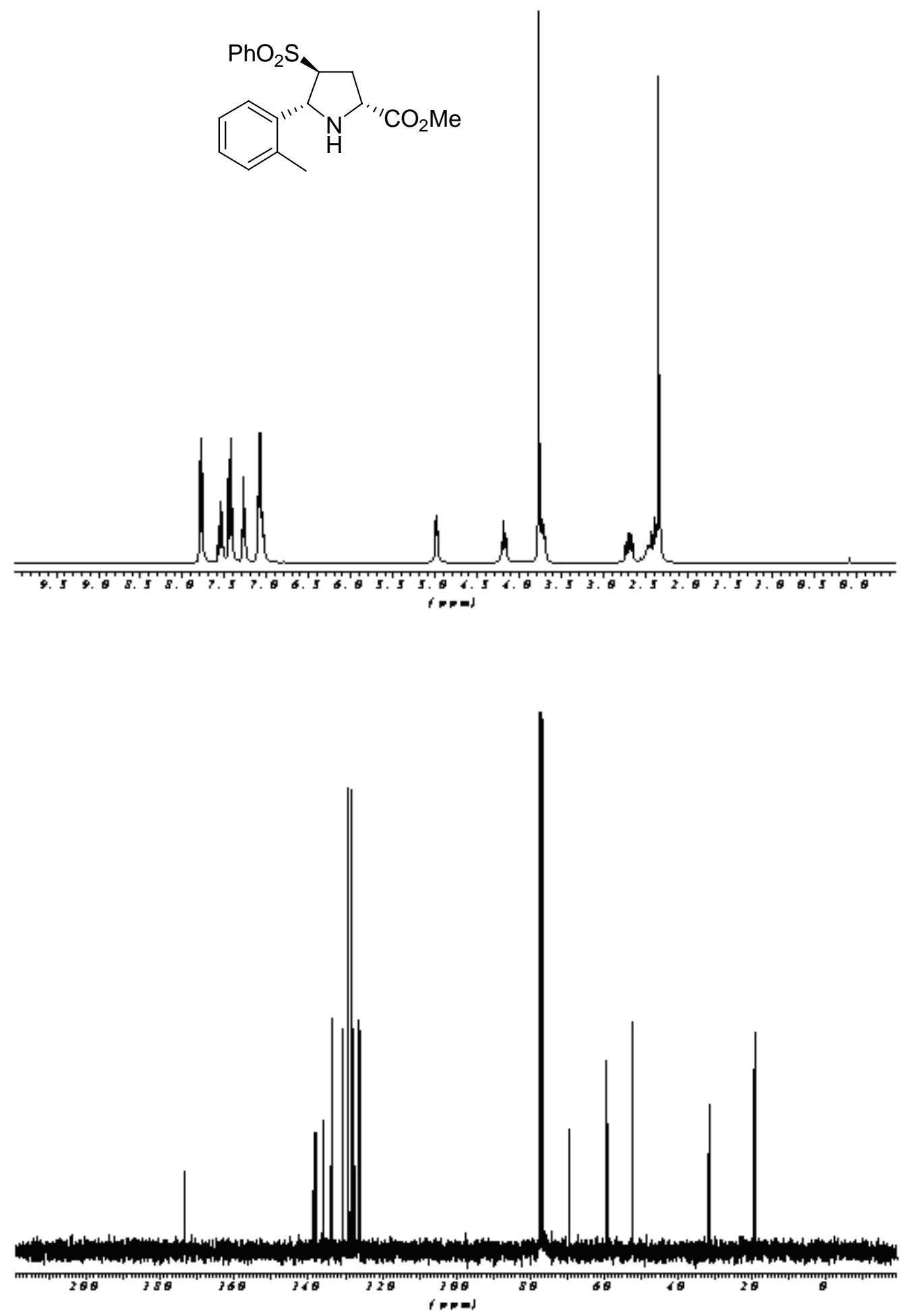

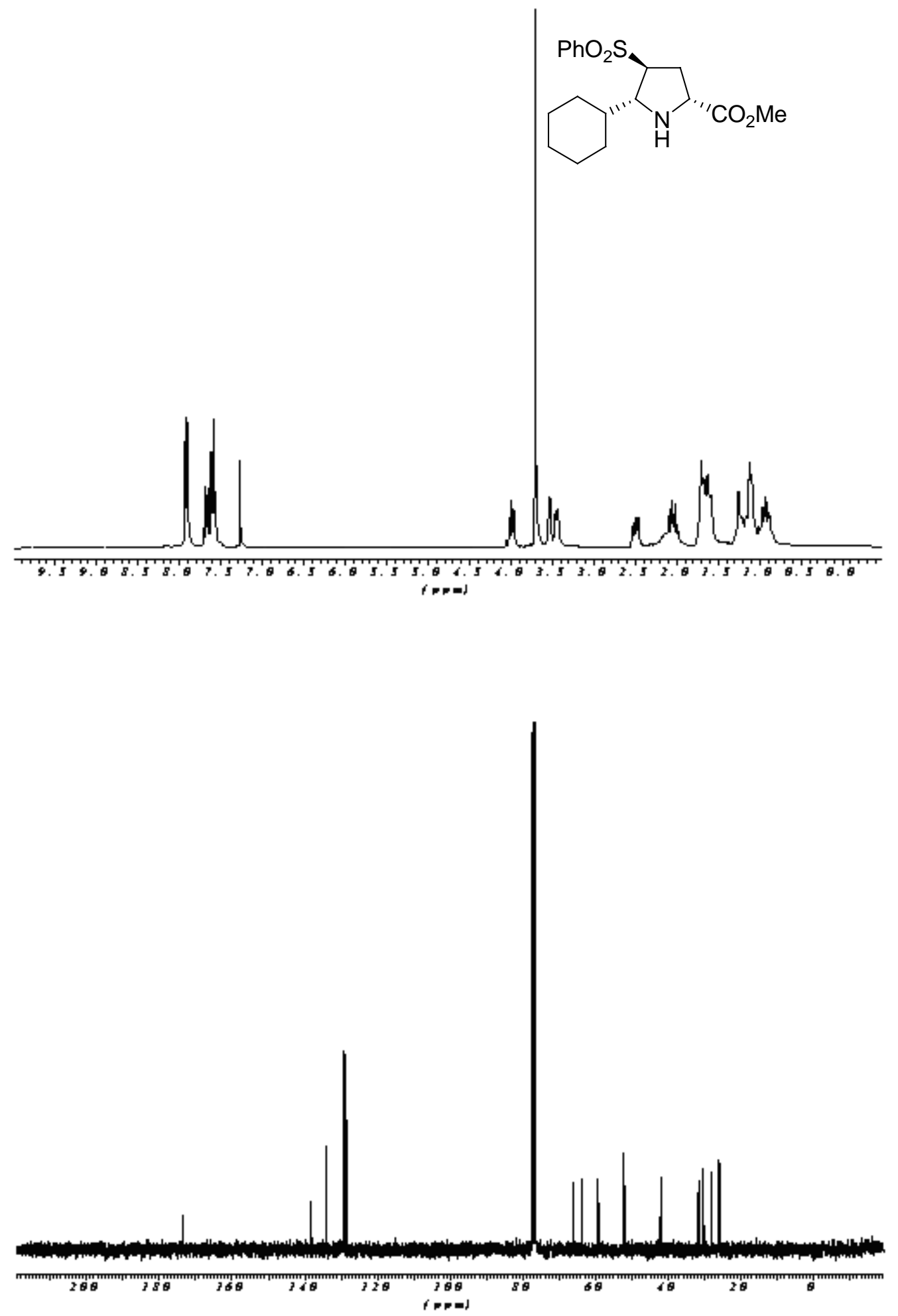

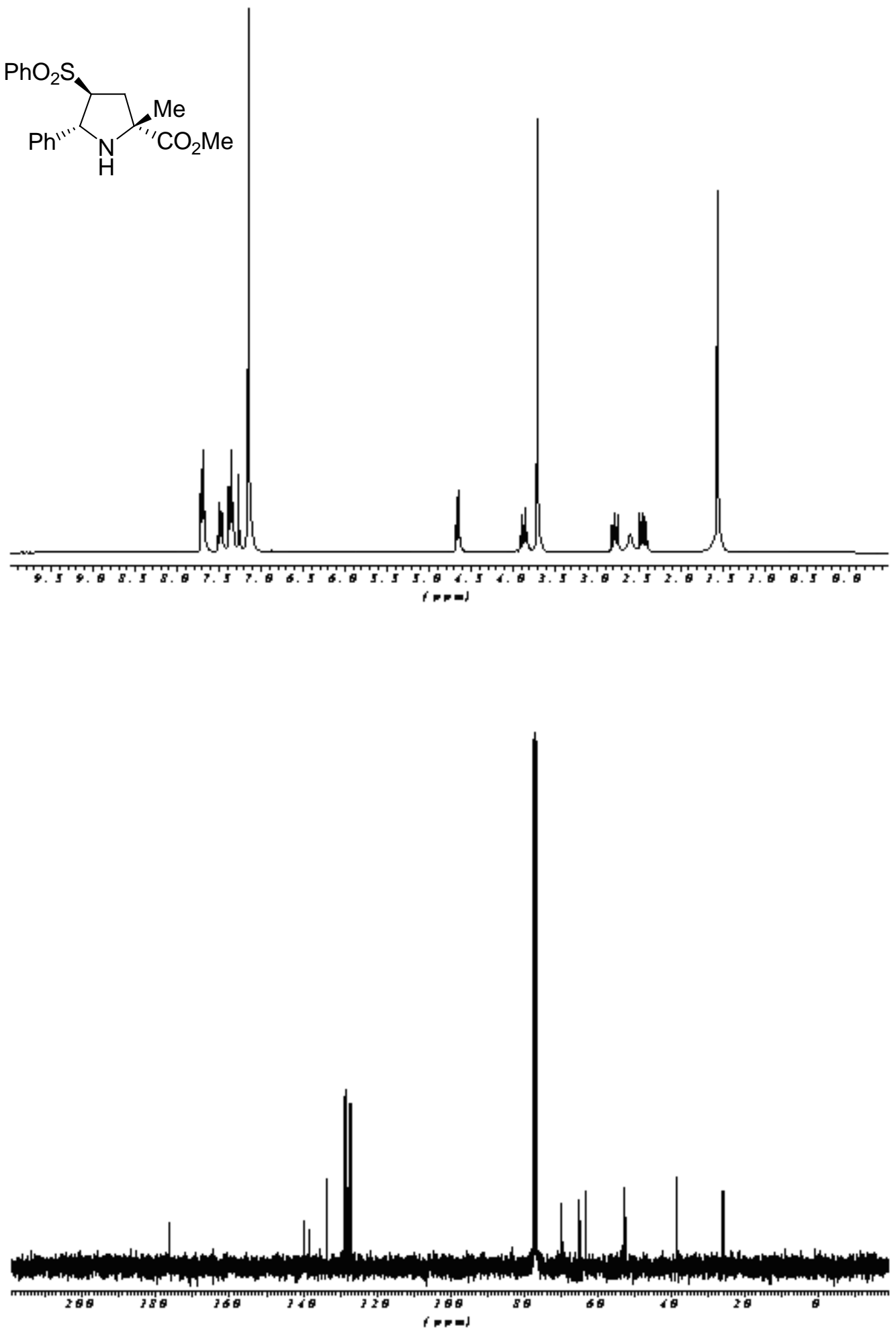

S 36 

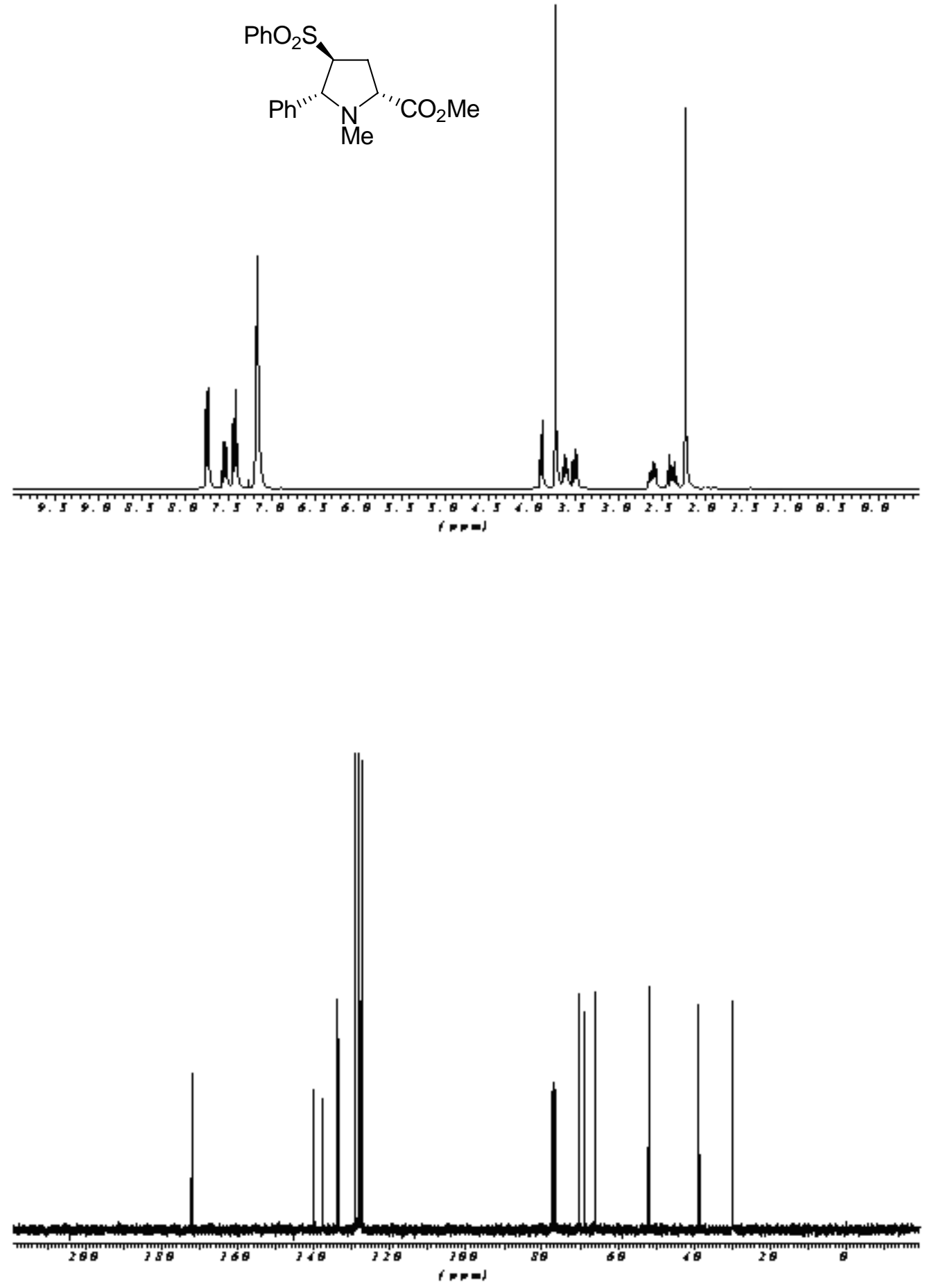

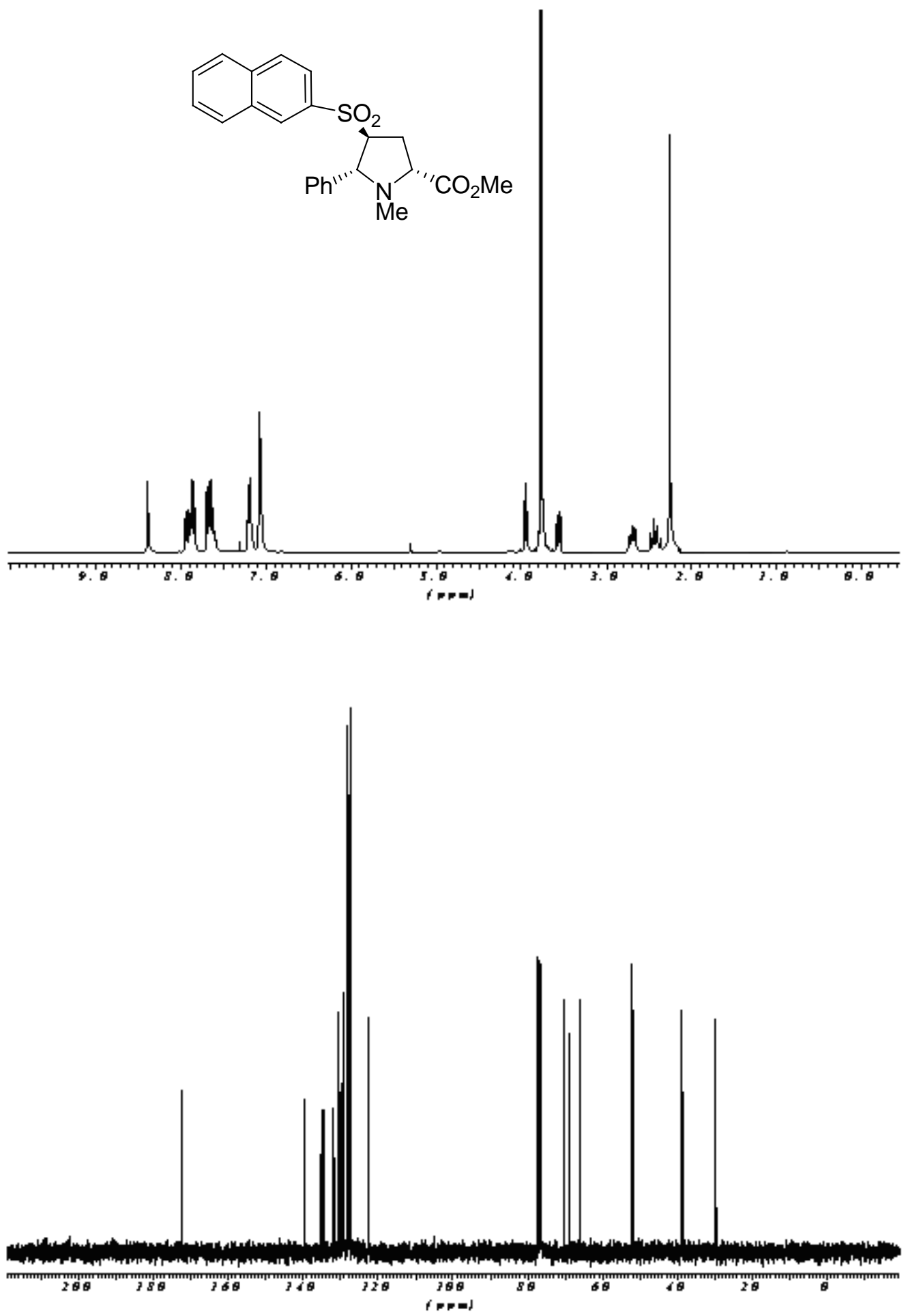

S 38 

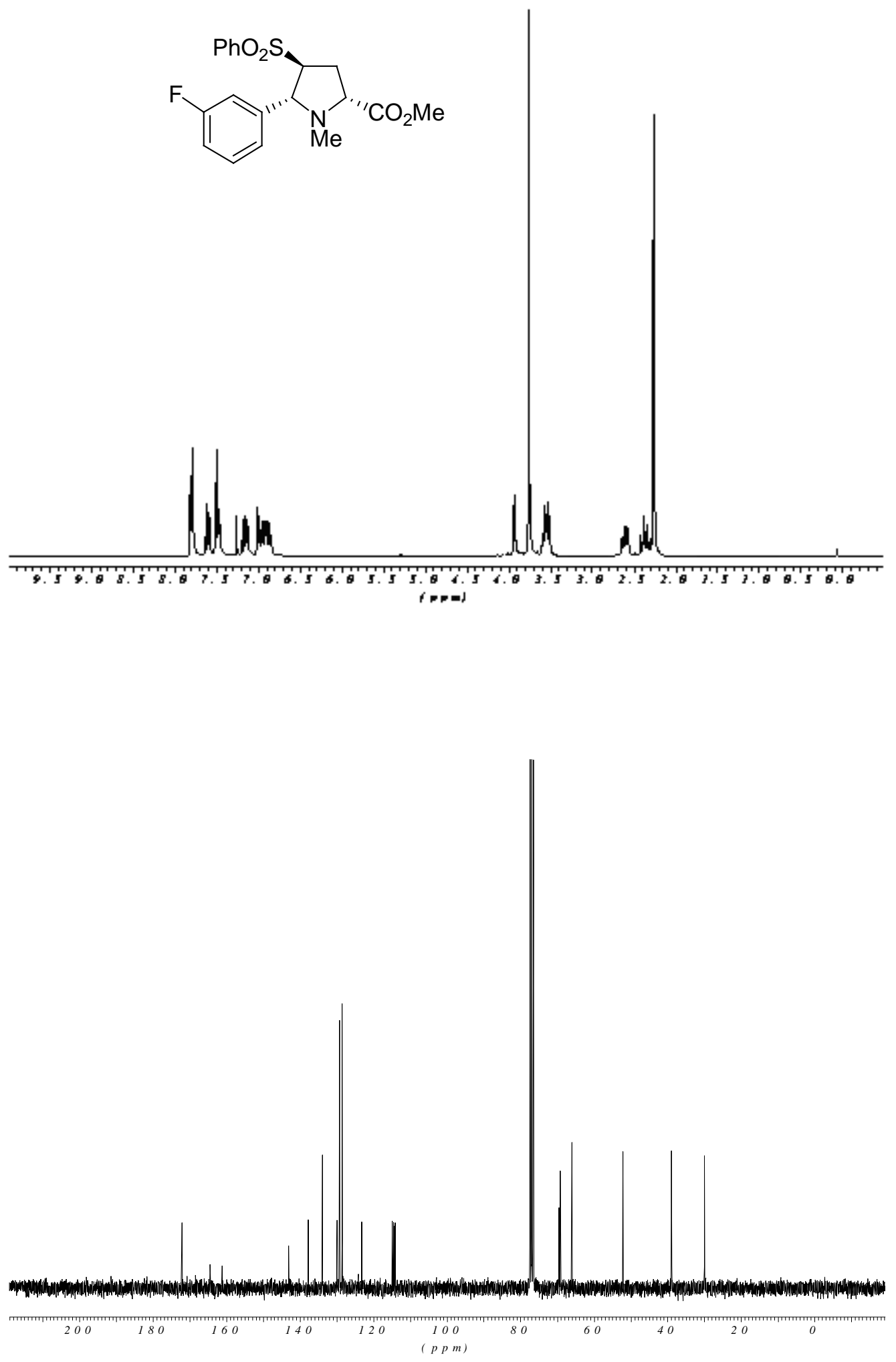

S 39 

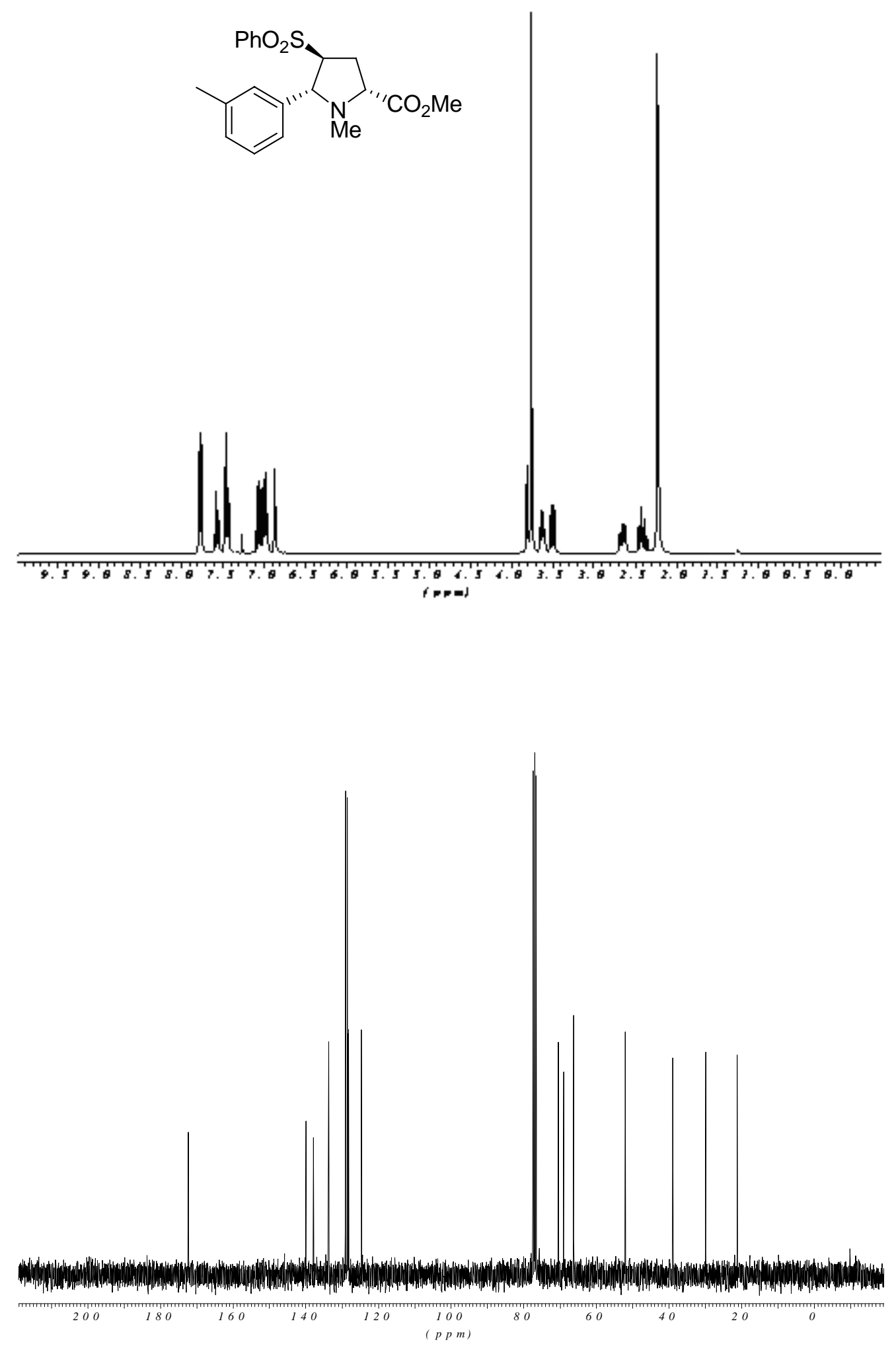

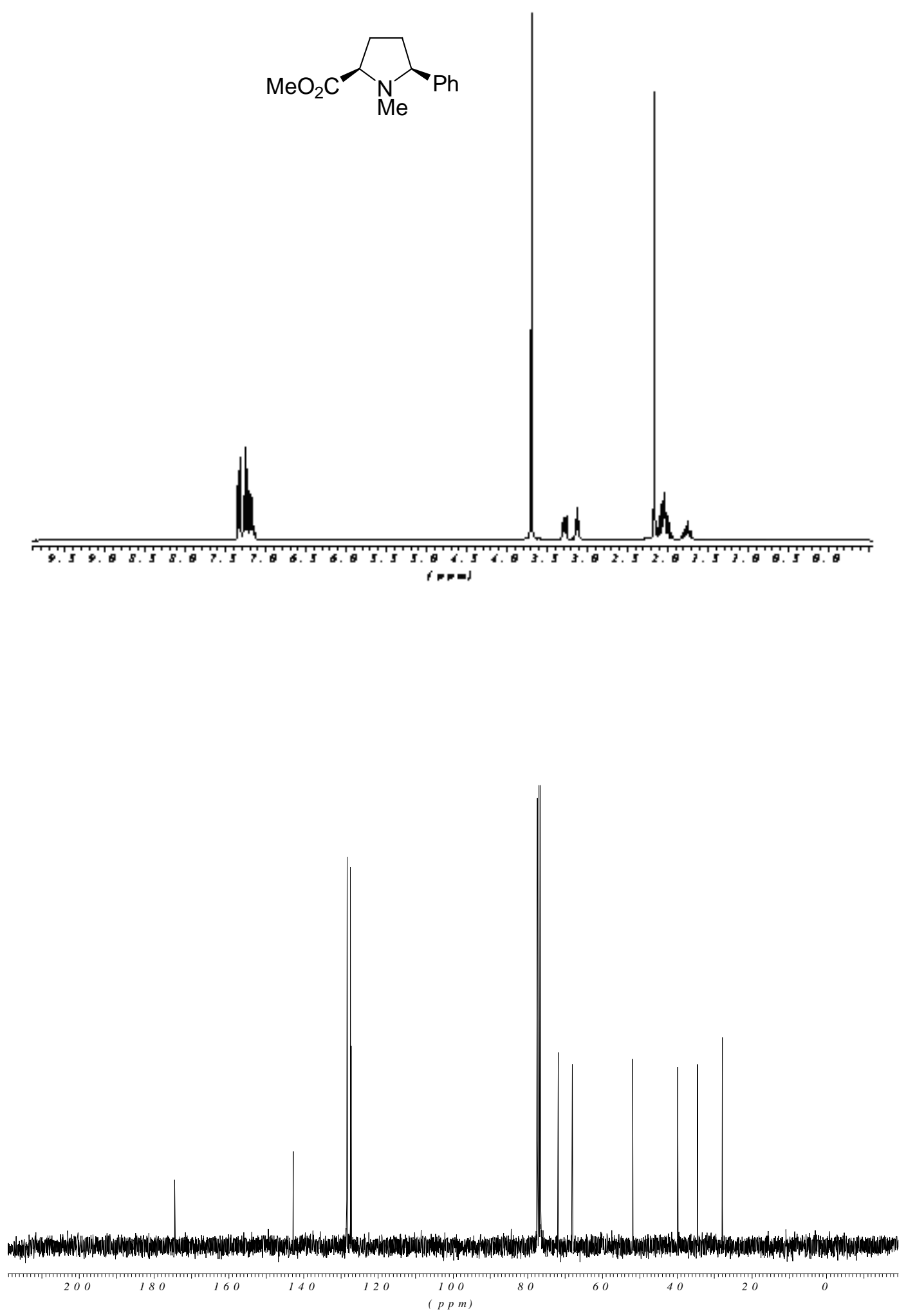

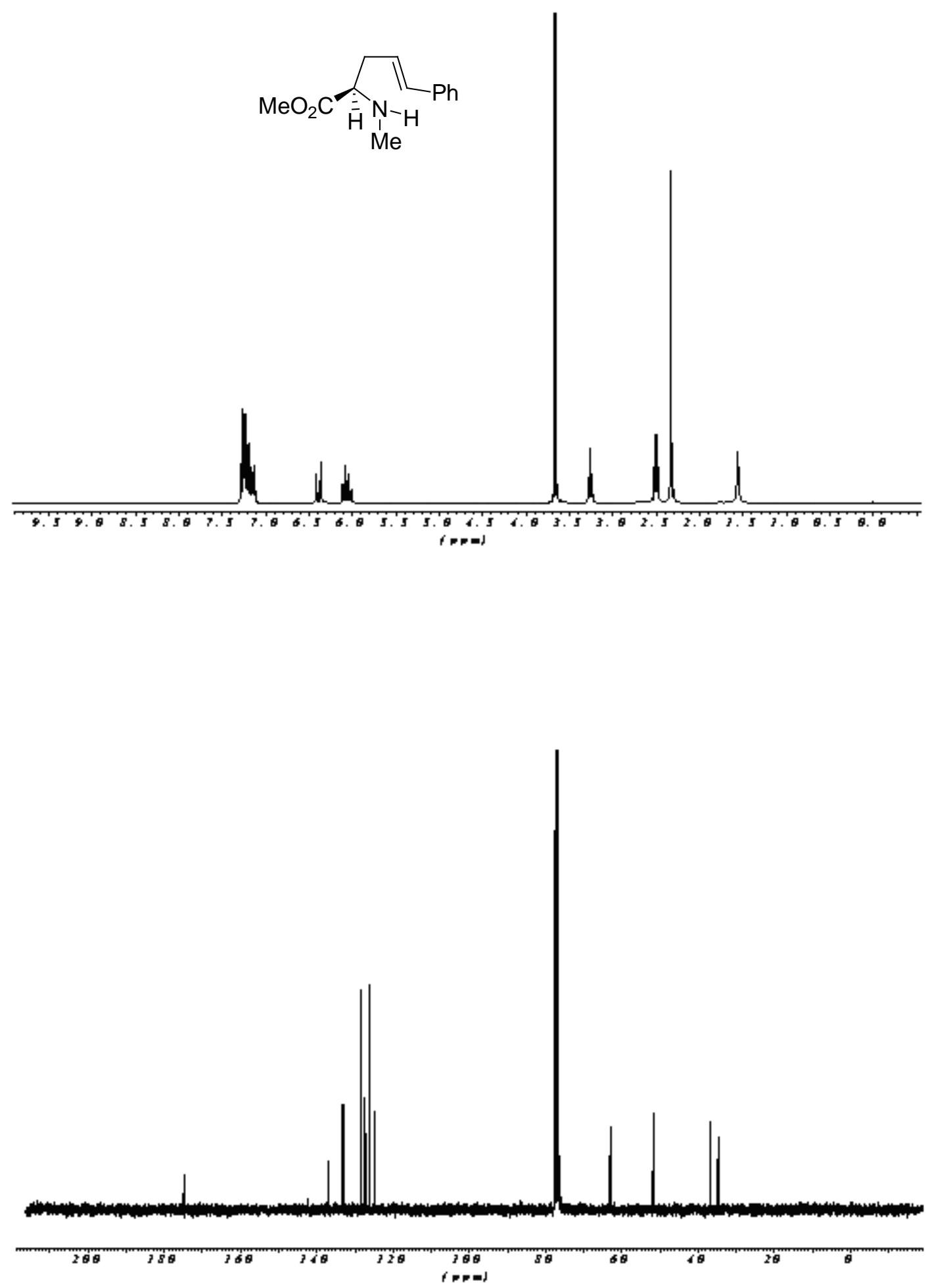


\section{X-Ray Analysis of exo-3a}

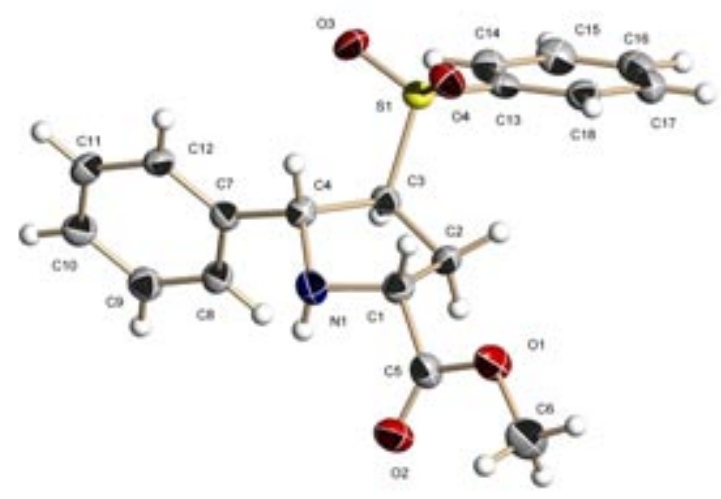

Table 1. Crystal data and structure refinement for exo-3a.

Empirical formula

Formula weight

Temperature

Wavelength

Crystal system

Space group

Unit cell dimensions

Volume

$\mathrm{Z}$

Density (calculated)

Absorption coefficient

$\mathrm{F}(000)$

Crystal size

Theta range for data collection

Index ranges

Reflections collected

Independent reflections

Completeness to theta $=71.41^{\circ}$

Absorption correction
C18 H19 N O4 S

345.40

100(2) K

$1.54178 \AA$

Orthorhombic

P2(1)2(1)2(1)

$a=8.5842(9) \AA \quad \alpha=90^{\circ}$.

$\mathrm{b}=13.1039(12) \AA \quad \beta=90^{\circ}$.

$\mathrm{c}=15.2066(17) \AA \quad \gamma=90^{\circ}$.

$1710.5(3) \AA^{3}$

4

$1.341 \mathrm{Mg} / \mathrm{m}^{3}$

$1.868 \mathrm{~mm}^{-1}$

728

$0.20 \times 0.05 \times 0.03 \mathrm{~mm}^{3}$

4.45 to $71.41^{\circ}$.

$-9<=\mathrm{h}<=10,-15<=\mathrm{k}<=15,-18<=\mathrm{l}<=17$

10897

3244 [R(int $)=0.0635]$

$98.7 \%$

Semi-empirical from equivalents 
Refinement method

Data / restraints / parameters

Goodness-of-fit on $\mathrm{F}^{2}$

Final $\mathrm{R}$ indices [I $>2 \operatorname{sigma}(\mathrm{I})]$

$\mathrm{R}$ indices (all data)

Absolute structure parameter

Largest diff. peak and hole

RUTINA. H MIXTOS.
Full-matrix least-squares on $\mathrm{F}^{2}$

3244 / 0 / 222

1.007

$\mathrm{R} 1=0.0434, \mathrm{wR} 2=0.0950$

$\mathrm{R} 1=0.0597, \mathrm{wR} 2=0.1030$

$-0.01(2)$

0.305 and -0.255 e. $\AA^{-3}$ 
Table 2. Atomic coordinates ( $\times 10^{4}$ ) and equivalent isotropic displacement parameters $\left(\AA^{2} \times 10^{3}\right)$ for exo-3a. U(eq) is defined as one third of the trace of the orthogonalized Uij tensor.

\begin{tabular}{lrrrr}
\hline & $\mathrm{x}$ & $\mathrm{y}$ & $\mathrm{z}$ & $\mathrm{U}(\mathrm{eq})$ \\
\hline $\mathrm{S}(1)$ & $2256(1)$ & $7712(1)$ & $7590(1)$ & $26(1)$ \\
$\mathrm{C}(1)$ & $4283(4)$ & $6152(2)$ & $9159(2)$ & $29(1)$ \\
$\mathrm{C}(2)$ & $2549(4)$ & $6370(2)$ & $8974(2)$ & $29(1)$ \\
$\mathrm{C}(3)$ & $2553(3)$ & $7503(2)$ & $8733(2)$ & $24(1)$ \\
$\mathrm{C}(4)$ & $4210(3)$ & $7903(2)$ & $8996(2)$ & $26(1)$ \\
$\mathrm{C}(5)$ & $4498(4)$ & $5259(2)$ & $9771(2)$ & $31(1)$ \\
$\mathrm{C}(6)$ & $4667(6)$ & $3462(3)$ & $9828(3)$ & $58(1)$ \\
$\mathrm{C}(7)$ & $4263(3)$ & $8918(2)$ & $9455(2)$ & $25(1)$ \\
$\mathrm{C}(8)$ & $3479(3)$ & $9055(2)$ & $10249(2)$ & $28(1)$ \\
$\mathrm{C}(9)$ & $3604(4)$ & $9966(2)$ & $10701(2)$ & $33(1)$ \\
$\mathrm{C}(10)$ & $4500(4)$ & $10759(2)$ & $10369(2)$ & $35(1)$ \\
$\mathrm{C}(11)$ & $5273(4)$ & $10630(2)$ & $9583(2)$ & $34(1)$ \\
$\mathrm{C}(12)$ & $5150(3)$ & $9715(2)$ & $9126(2)$ & $28(1)$ \\
$\mathrm{C}(13)$ & $267(3)$ & $7427(2)$ & $7409(2)$ & $27(1)$ \\
$\mathrm{C}(14)$ & $-818(3)$ & $8200(2)$ & $7525(2)$ & $32(1)$ \\
$\mathrm{C}(15)$ & $-2374(4)$ & $7981(2)$ & $7362(2)$ & $36(1)$ \\
$\mathrm{C}(16)$ & $-2820(4)$ & $7009(3)$ & $7103(2)$ & $38(1)$ \\
$\mathrm{C}(17)$ & $-1718(4)$ & $6251(3)$ & $6988(2)$ & $34(1)$ \\
$\mathrm{C}(18)$ & $-155(4)$ & $6461(2)$ & $7137(2)$ & $31(1)$ \\
$\mathrm{N}(1)$ & $4906(3)$ & $7085(2)$ & $9530(2)$ & $30(1)$ \\
$\mathrm{O}(1)$ & $4478(3)$ & $4390(2)$ & $9320(2)$ & $43(1)$ \\
$\mathrm{O}(2)$ & $4640(3)$ & $5331(2)$ & $10555(2)$ & $39(1)$ \\
$\mathrm{O}(3)$ & $2508(2)$ & $8777(1)$ & $7429(2)$ & $37(1)$ \\
$\mathrm{O}(4)$ & $3173(2)$ & $6984(2)$ & $7107(1)$ & $33(1)$ \\
- & & & \\
\hline & & & &
\end{tabular}


Table 3. Bond lengths $[\AA]$ and angles $\left[{ }^{\circ}\right]$ for exo-3a.

\begin{tabular}{ll}
\hline $\mathrm{S}(1)-\mathrm{O}(3)$ & $1.433(2)$ \\
$\mathrm{S}(1)-\mathrm{O}(4)$ & $1.439(2)$ \\
$\mathrm{S}(1)-\mathrm{C}(13)$ & $1.769(3)$ \\
$\mathrm{S}(1)-\mathrm{C}(3)$ & $1.779(3)$ \\
$\mathrm{C}(1)-\mathrm{N}(1)$ & $1.449(4)$ \\
$\mathrm{C}(1)-\mathrm{C}(5)$ & $1.506(4)$ \\
$\mathrm{C}(1)-\mathrm{C}(2)$ & $1.542(4)$ \\
$\mathrm{C}(1)-\mathrm{H}(1)$ & 1.0000 \\
$\mathrm{C}(2)-\mathrm{C}(3)$ & $1.530(4)$ \\
$\mathrm{C}(2)-\mathrm{H}(2 \mathrm{~A})$ & 0.9900 \\
$\mathrm{C}(2)-\mathrm{H}(2 \mathrm{~B})$ & 0.9900 \\
$\mathrm{C}(3)-\mathrm{C}(4)$ & $1.567(4)$ \\
$\mathrm{C}(3)-\mathrm{H}(3)$ & 1.0000 \\
$\mathrm{C}(4)-\mathrm{N}(1)$ & $1.472(4)$ \\
$\mathrm{C}(4)-\mathrm{C}(7)$ & $1.502(4)$ \\
$\mathrm{C}(4)-\mathrm{H}(4)$ & 1.0000 \\
$\mathrm{C}(5)-\mathrm{O}(2)$ & $1.202(4)$ \\
$\mathrm{C}(5)-\mathrm{O}(1)$ & $1.330(4)$ \\
$\mathrm{C}(6)-\mathrm{O}(1)$ & $1.449(4)$ \\
$\mathrm{C}(6)-\mathrm{H}(6 \mathrm{~A})$ & 0.9800 \\
$\mathrm{C}(6)-\mathrm{H}(6 \mathrm{~B})$ & 0.9800 \\
$\mathrm{C}(6)-\mathrm{H}(6 \mathrm{C})$ & 0.9800 \\
$\mathrm{C}(7)-\mathrm{C}(12)$ & $1.386(4)$ \\
$\mathrm{C}(7)-\mathrm{C}(8)$ & $1.395(4)$ \\
$\mathrm{C}(8)-\mathrm{C}(9)$ & $1.381(4)$ \\
$\mathrm{C}(8)-\mathrm{H}(8)$ & 0.9500 \\
$\mathrm{C}(9)-\mathrm{C}(10)$ & $1.388(5)$ \\
$\mathrm{C}(9)-\mathrm{H}(9)$ & 0.9500 \\
$\mathrm{C}(10)-\mathrm{C}(11)$ & $1.378(5)$ \\
$\mathrm{C}(10)-\mathrm{H}(10)$ & 0.9500 \\
$\mathrm{C}(11)-\mathrm{C}(12)$ & $1.389(4)$ \\
$\mathrm{C}(11)-\mathrm{H}(11)$ & 0.9500 \\
$\mathrm{C}(12)-\mathrm{H}(12)$ & 0.9500 \\
$\mathrm{C}(13)-\mathrm{C}(18)$ & $1.380(4)$ \\
&
\end{tabular}




\begin{tabular}{|c|c|}
\hline$C(13)-C(14)$ & $1.387(4)$ \\
\hline $\mathrm{C}(14)-\mathrm{C}(15)$ & $1.389(4)$ \\
\hline $\mathrm{C}(14)-\mathrm{H}(14)$ & 0.9500 \\
\hline$C(15)-C(16)$ & $1.387(4)$ \\
\hline $\mathrm{C}(15)-\mathrm{H}(15)$ & 0.9500 \\
\hline$C(16)-C(17)$ & $1.383(5)$ \\
\hline $\mathrm{C}(16)-\mathrm{H}(16)$ & 0.9500 \\
\hline $\mathrm{C}(17)-\mathrm{C}(18)$ & $1.388(5)$ \\
\hline $\mathrm{C}(17)-\mathrm{H}(17)$ & 0.9500 \\
\hline $\mathrm{C}(18)-\mathrm{H}(18)$ & 0.9500 \\
\hline $\mathrm{N}(1)-\mathrm{H}(1 \mathrm{~A})$ & $0.87(4)$ \\
\hline $\mathrm{O}(3)-\mathrm{S}(1)-\mathrm{O}(4)$ & $118.47(14)$ \\
\hline $\mathrm{O}(3)-\mathrm{S}(1)-\mathrm{C}(13)$ & $108.93(13)$ \\
\hline $\mathrm{O}(4)-\mathrm{S}(1)-\mathrm{C}(13)$ & $108.00(13)$ \\
\hline $\mathrm{O}(3)-\mathrm{S}(1)-\mathrm{C}(3)$ & $107.13(13)$ \\
\hline $\mathrm{O}(4)-\mathrm{S}(1)-\mathrm{C}(3)$ & $108.58(13)$ \\
\hline $\mathrm{C}(13)-\mathrm{S}(1)-\mathrm{C}(3)$ & $104.93(13)$ \\
\hline $\mathrm{N}(1)-\mathrm{C}(1)-\mathrm{C}(5)$ & $111.7(3)$ \\
\hline $\mathrm{N}(1)-\mathrm{C}(1)-\mathrm{C}(2)$ & $105.7(2)$ \\
\hline $\mathrm{C}(5)-\mathrm{C}(1)-\mathrm{C}(2)$ & $112.0(3)$ \\
\hline $\mathrm{N}(1)-\mathrm{C}(1)-\mathrm{H}(1)$ & 109.1 \\
\hline $\mathrm{C}(5)-\mathrm{C}(1)-\mathrm{H}(1)$ & 109.1 \\
\hline $\mathrm{C}(2)-\mathrm{C}(1)-\mathrm{H}(1)$ & 109.1 \\
\hline$C(3)-C(2)-C(1)$ & $102.8(2)$ \\
\hline $\mathrm{C}(3)-\mathrm{C}(2)-\mathrm{H}(2 \mathrm{~A})$ & 111.2 \\
\hline $\mathrm{C}(1)-\mathrm{C}(2)-\mathrm{H}(2 \mathrm{~A})$ & 111.2 \\
\hline $\mathrm{C}(3)-\mathrm{C}(2)-\mathrm{H}(2 \mathrm{~B})$ & 111.2 \\
\hline $\mathrm{C}(1)-\mathrm{C}(2)-\mathrm{H}(2 \mathrm{~B})$ & 111.2 \\
\hline $\mathrm{H}(2 \mathrm{~A})-\mathrm{C}(2)-\mathrm{H}(2 \mathrm{~B})$ & 109.1 \\
\hline $\mathrm{C}(2)-\mathrm{C}(3)-\mathrm{C}(4)$ & $105.4(2)$ \\
\hline $\mathrm{C}(2)-\mathrm{C}(3)-\mathrm{S}(1)$ & $112.53(19)$ \\
\hline $\mathrm{C}(4)-\mathrm{C}(3)-\mathrm{S}(1)$ & $109.14(18)$ \\
\hline $\mathrm{C}(2)-\mathrm{C}(3)-\mathrm{H}(3)$ & 109.9 \\
\hline $\mathrm{C}(4)-\mathrm{C}(3)-\mathrm{H}(3)$ & 109.9 \\
\hline $\mathrm{S}(1)-\mathrm{C}(3)-\mathrm{H}(3)$ & 109.9 \\
\hline
\end{tabular}




\begin{tabular}{|c|c|}
\hline $\mathrm{N}(1)-\mathrm{C}(4)-\mathrm{C}(7)$ & $112.1(2)$ \\
\hline $\mathrm{N}(1)-\mathrm{C}(4)-\mathrm{C}(3)$ & $105.4(2)$ \\
\hline$C(7)-C(4)-C(3)$ & $116.2(2)$ \\
\hline $\mathrm{N}(1)-\mathrm{C}(4)-\mathrm{H}(4)$ & 107.6 \\
\hline $\mathrm{C}(7)-\mathrm{C}(4)-\mathrm{H}(4)$ & 107.6 \\
\hline $\mathrm{C}(3)-\mathrm{C}(4)-\mathrm{H}(4)$ & 107.6 \\
\hline $\mathrm{O}(2)-\mathrm{C}(5)-\mathrm{O}(1)$ & $125.3(3)$ \\
\hline $\mathrm{O}(2)-\mathrm{C}(5)-\mathrm{C}(1)$ & $124.4(3)$ \\
\hline $\mathrm{O}(1)-\mathrm{C}(5)-\mathrm{C}(1)$ & $110.3(3)$ \\
\hline $\mathrm{O}(1)-\mathrm{C}(6)-\mathrm{H}(6 \mathrm{~A})$ & 109.5 \\
\hline $\mathrm{O}(1)-\mathrm{C}(6)-\mathrm{H}(6 \mathrm{~B})$ & 109.5 \\
\hline $\mathrm{H}(6 \mathrm{~A})-\mathrm{C}(6)-\mathrm{H}(6 \mathrm{~B})$ & 109.5 \\
\hline $\mathrm{O}(1)-\mathrm{C}(6)-\mathrm{H}(6 \mathrm{C})$ & 109.5 \\
\hline $\mathrm{H}(6 \mathrm{~A})-\mathrm{C}(6)-\mathrm{H}(6 \mathrm{C})$ & 109.5 \\
\hline $\mathrm{H}(6 \mathrm{~B})-\mathrm{C}(6)-\mathrm{H}(6 \mathrm{C})$ & 109.5 \\
\hline $\mathrm{C}(12)-\mathrm{C}(7)-\mathrm{C}(8)$ & $118.7(3)$ \\
\hline$C(12)-C(7)-C(4)$ & $121.1(3)$ \\
\hline$C(8)-C(7)-C(4)$ & $120.1(3)$ \\
\hline $\mathrm{C}(9)-\mathrm{C}(8)-\mathrm{C}(7)$ & $120.3(3)$ \\
\hline $\mathrm{C}(9)-\mathrm{C}(8)-\mathrm{H}(8)$ & 119.8 \\
\hline $\mathrm{C}(7)-\mathrm{C}(8)-\mathrm{H}(8)$ & 119.8 \\
\hline $\mathrm{C}(8)-\mathrm{C}(9)-\mathrm{C}(10)$ & $120.6(3)$ \\
\hline $\mathrm{C}(8)-\mathrm{C}(9)-\mathrm{H}(9)$ & 119.7 \\
\hline $\mathrm{C}(10)-\mathrm{C}(9)-\mathrm{H}(9)$ & 119.7 \\
\hline $\mathrm{C}(11)-\mathrm{C}(10)-\mathrm{C}(9)$ & $119.4(3)$ \\
\hline $\mathrm{C}(11)-\mathrm{C}(10)-\mathrm{H}(10)$ & 120.3 \\
\hline $\mathrm{C}(9)-\mathrm{C}(10)-\mathrm{H}(10)$ & 120.3 \\
\hline $\mathrm{C}(10)-\mathrm{C}(11)-\mathrm{C}(12)$ & $120.2(3)$ \\
\hline $\mathrm{C}(10)-\mathrm{C}(11)-\mathrm{H}(11)$ & 119.9 \\
\hline $\mathrm{C}(12)-\mathrm{C}(11)-\mathrm{H}(11)$ & 119.9 \\
\hline $\mathrm{C}(7)-\mathrm{C}(12)-\mathrm{C}(11)$ & $120.8(3)$ \\
\hline $\mathrm{C}(7)-\mathrm{C}(12)-\mathrm{H}(12)$ & 119.6 \\
\hline $\mathrm{C}(11)-\mathrm{C}(12)-\mathrm{H}(12)$ & 119.6 \\
\hline $\mathrm{C}(18)-\mathrm{C}(13)-\mathrm{C}(14)$ & $122.1(3)$ \\
\hline $\mathrm{C}(18)-\mathrm{C}(13)-\mathrm{S}(1)$ & $119.5(2)$ \\
\hline$C(14)-C(13)-S(1)$ & $118.3(2)$ \\
\hline
\end{tabular}




$\begin{array}{ll}\mathrm{C}(13)-\mathrm{C}(14)-\mathrm{C}(15) & 118.2(3) \\ \mathrm{C}(13)-\mathrm{C}(14)-\mathrm{H}(14) & 120.9 \\ \mathrm{C}(15)-\mathrm{C}(14)-\mathrm{H}(14) & 120.9 \\ \mathrm{C}(16)-\mathrm{C}(15)-\mathrm{C}(14) & 120.4(3) \\ \mathrm{C}(16)-\mathrm{C}(15)-\mathrm{H}(15) & 119.8 \\ \mathrm{C}(14)-\mathrm{C}(15)-\mathrm{H}(15) & 119.8 \\ \mathrm{C}(17)-\mathrm{C}(16)-\mathrm{C}(15) & 120.4(3) \\ \mathrm{C}(17)-\mathrm{C}(16)-\mathrm{H}(16) & 119.8 \\ \mathrm{C}(15)-\mathrm{C}(16)-\mathrm{H}(16) & 119.8 \\ \mathrm{C}(16)-\mathrm{C}(17)-\mathrm{C}(18) & 119.9(3) \\ \mathrm{C}(16)-\mathrm{C}(17)-\mathrm{H}(17) & 120.0 \\ \mathrm{C}(18)-\mathrm{C}(17)-\mathrm{H}(17) & 120.0 \\ \mathrm{C}(13)-\mathrm{C}(18)-\mathrm{C}(17) & 119.0(3) \\ \mathrm{C}(13)-\mathrm{C}(18)-\mathrm{H}(18) & 120.5 \\ \mathrm{C}(17)-\mathrm{C}(18)-\mathrm{H}(18) & 120.5 \\ \mathrm{C}(1)-\mathrm{N}(1)-\mathrm{C}(4) & 104.5(2) \\ \mathrm{C}(1)-\mathrm{N}(1)-\mathrm{H}(1 \mathrm{~A}) & 108(3) \\ \mathrm{C}(4)-\mathrm{N}(1)-\mathrm{H}(1 \mathrm{~A}) & 108(3) \\ \mathrm{C}(5)-\mathrm{O}(1)-\mathrm{C}(6) & 116.3(3) \\ & \end{array}$


Table 4. Anisotropic displacement parameters $\left(\AA^{2} \times 10^{3}\right)$ for exo-3a. The anisotropic displacement factor exponent takes the form: $-2 \pi^{2}\left[h^{2} a^{* 2} U^{11}+\ldots+2 h \mathrm{k}^{*} b^{*} U^{12}\right]$

\begin{tabular}{lcccccc}
\hline & $\mathrm{U}^{11}$ & $\mathrm{U}^{22}$ & $\mathrm{U}^{33}$ & $\mathrm{U}^{23}$ & $\mathrm{U}^{13}$ & $\mathrm{U} 12$ \\
\hline $\mathrm{S}(1)$ & $27(1)$ & $27(1)$ & $23(1)$ & $2(1)$ & $-1(1)$ & $0(1)$ \\
$\mathrm{C}(1)$ & $32(2)$ & $24(2)$ & $30(2)$ & $-1(1)$ & $1(1)$ & $2(1)$ \\
$\mathrm{C}(2)$ & $31(2)$ & $28(2)$ & $30(2)$ & $7(1)$ & $-5(1)$ & $-6(1)$ \\
$\mathrm{C}(3)$ & $23(2)$ & $29(2)$ & $21(1)$ & $-2(1)$ & $1(1)$ & $0(1)$ \\
$\mathrm{C}(4)$ & $25(2)$ & $24(2)$ & $29(2)$ & $-1(1)$ & $2(1)$ & $1(1)$ \\
$\mathrm{C}(5)$ & $31(2)$ & $27(2)$ & $35(2)$ & $-3(1)$ & $-4(1)$ & $5(1)$ \\
$\mathrm{C}(6)$ & $94(3)$ & $23(2)$ & $57(2)$ & $1(2)$ & $-27(2)$ & $9(2)$ \\
$\mathrm{C}(7)$ & $26(2)$ & $22(1)$ & $25(2)$ & $-1(1)$ & $-3(1)$ & $4(1)$ \\
$\mathrm{C}(8)$ & $26(2)$ & $26(2)$ & $32(2)$ & $0(1)$ & $1(1)$ & $0(1)$ \\
$\mathrm{C}(9)$ & $36(2)$ & $34(2)$ & $28(2)$ & $-3(1)$ & $3(1)$ & $1(1)$ \\
$\mathrm{C}(10)$ & $51(2)$ & $23(2)$ & $31(2)$ & $-2(1)$ & $-2(2)$ & $2(2)$ \\
$\mathrm{C}(11)$ & $44(2)$ & $23(2)$ & $36(2)$ & $5(1)$ & $-3(2)$ & $-4(1)$ \\
$\mathrm{C}(12)$ & $33(2)$ & $26(2)$ & $24(2)$ & $2(1)$ & $0(1)$ & $-1(1)$ \\
$\mathrm{C}(13)$ & $31(2)$ & $30(2)$ & $20(1)$ & $4(1)$ & $-2(1)$ & $1(1)$ \\
$\mathrm{C}(14)$ & $37(2)$ & $29(2)$ & $29(2)$ & $-4(1)$ & $1(2)$ & $4(1)$ \\
$\mathrm{C}(15)$ & $30(2)$ & $44(2)$ & $35(2)$ & $-2(1)$ & $3(1)$ & $9(1)$ \\
$\mathrm{C}(16)$ & $28(2)$ & $55(2)$ & $29(2)$ & $3(1)$ & $1(1)$ & $-4(2)$ \\
$\mathrm{C}(17)$ & $35(2)$ & $35(2)$ & $32(2)$ & $1(1)$ & $-4(1)$ & $-7(1)$ \\
$\mathrm{C}(18)$ & $36(2)$ & $28(2)$ & $29(2)$ & $3(1)$ & $-2(1)$ & $3(1)$ \\
$\mathrm{N}(1)$ & $30(1)$ & $26(1)$ & $34(2)$ & $-3(1)$ & $-8(1)$ & $4(1)$ \\
$\mathrm{O}(1)$ & $67(2)$ & $22(1)$ & $39(1)$ & $-3(1)$ & $-14(1)$ & $6(1)$ \\
$\mathrm{O}(2)$ & $52(2)$ & $34(1)$ & $31(1)$ & $-1(1)$ & $-7(1)$ & $8(1)$ \\
$\mathrm{O}(3)$ & $40(1)$ & $32(1)$ & $39(1)$ & $14(1)$ & $-5(1)$ & $-7(1)$ \\
$\mathrm{O}(4)$ & $29(1)$ & $43(1)$ & $27(1)$ & $-6(1)$ & $1(1)$ & $3(1)$ \\
& & & & & & \\
\hline
\end{tabular}


Table 5. Hydrogen coordinates ( $\left.\times 10^{4}\right)$ and isotropic displacement parameters $\left(\AA^{2} \times 10\right.$ 3) for exo-3a.

\begin{tabular}{lcccl}
\hline & \multicolumn{1}{c}{$\mathrm{x}$} & $\mathrm{z}$ & $\mathrm{U}(\mathrm{eq})$ \\
\hline & & & & \\
$\mathrm{H}(1)$ & 4825 & 6005 & 8591 & 34 \\
$\mathrm{H}(2 \mathrm{~A})$ & 2158 & 5948 & 8482 & 35 \\
$\mathrm{H}(2 \mathrm{~B})$ & 1902 & 6240 & 9502 & 35 \\
$\mathrm{H}(3)$ & 1737 & 7871 & 9079 & 29 \\
$\mathrm{H}(4)$ & 4838 & 7964 & 8444 & 31 \\
$\mathrm{H}(6 \mathrm{~A})$ & 5682 & 3471 & 10123 & 87 \\
$\mathrm{H}(6 \mathrm{~B})$ & 4611 & 2870 & 9435 & 87 \\
$\mathrm{H}(6 \mathrm{C})$ & 3837 & 3417 & 10269 & 87 \\
$\mathrm{H}(8)$ & 2856 & 8519 & 10481 & 33 \\
$\mathrm{H}(9)$ & 3071 & 10051 & 11244 & 39 \\
$\mathrm{H}(10)$ & 4580 & 11385 & 10681 & 42 \\
$\mathrm{H}(11)$ & 5891 & 11168 & 9352 & 41 \\
$\mathrm{H}(12)$ & 5680 & 9635 & 8582 & 33 \\
$\mathrm{H}(14)$ & -505 & 8861 & 7712 & 38 \\
$\mathrm{H}(15)$ & -3137 & 8500 & 7428 & 43 \\
$\mathrm{H}(16)$ & -3890 & 6863 & 7004 & 45 \\
$\mathrm{H}(17)$ & -2029 & 5588 & 6806 & 41 \\
$\mathrm{H}(18)$ & 613 & 5948 & 7054 & 37 \\
$\mathrm{H}(1 \mathrm{~A})$ & $4550(50)$ & $7150(30)$ & $10060(30)$ & $55(13)$ \\
- & & & & \\
\hline
\end{tabular}


Table 6. Torsion angles $\left[{ }^{\circ}\right]$ for exo-3a.

\begin{tabular}{lc}
\hline $\mathrm{N}(1)-\mathrm{C}(1)-\mathrm{C}(2)-\mathrm{C}(3)$ & $33.1(3)$ \\
$\mathrm{C}(5)-\mathrm{C}(1)-\mathrm{C}(2)-\mathrm{C}(3)$ & $154.9(2)$ \\
$\mathrm{C}(1)-\mathrm{C}(2)-\mathrm{C}(3)-\mathrm{C}(4)$ & $-12.0(3)$ \\
$\mathrm{C}(1)-\mathrm{C}(2)-\mathrm{C}(3)-\mathrm{S}(1)$ & $106.8(2)$ \\
$\mathrm{O}(3)-\mathrm{S}(1)-\mathrm{C}(3)-\mathrm{C}(2)$ & $-171.8(2)$ \\
$\mathrm{O}(4)-\mathrm{S}(1)-\mathrm{C}(3)-\mathrm{C}(2)$ & $-42.8(2)$ \\
$\mathrm{C}(13)-\mathrm{S}(1)-\mathrm{C}(3)-\mathrm{C}(2)$ & $72.5(2)$ \\
$\mathrm{O}(3)-\mathrm{S}(1)-\mathrm{C}(3)-\mathrm{C}(4)$ & $-55.2(2)$ \\
$\mathrm{O}(4)-\mathrm{S}(1)-\mathrm{C}(3)-\mathrm{C}(4)$ & $73.8(2)$ \\
$\mathrm{C}(13)-\mathrm{S}(1)-\mathrm{C}(3)-\mathrm{C}(4)$ & $-170.89(18)$ \\
$\mathrm{C}(2)-\mathrm{C}(3)-\mathrm{C}(4)-\mathrm{N}(1)$ & $-12.1(3)$ \\
$\mathrm{S}(1)-\mathrm{C}(3)-\mathrm{C}(4)-\mathrm{N}(1)$ & $-133.1(2)$ \\
$\mathrm{C}(2)-\mathrm{C}(3)-\mathrm{C}(4)-\mathrm{C}(7)$ & $-136.8(3)$ \\
$\mathrm{S}(1)-\mathrm{C}(3)-\mathrm{C}(4)-\mathrm{C}(7)$ & $102.1(2)$ \\
$\mathrm{N}(1)-\mathrm{C}(1)-\mathrm{C}(5)-\mathrm{O}(2)$ & $24.5(5)$ \\
$\mathrm{C}(2)-\mathrm{C}(1)-\mathrm{C}(5)-\mathrm{O}(2)$ & $-93.9(4)$ \\
$\mathrm{N}(1)-\mathrm{C}(1)-\mathrm{C}(5)-\mathrm{O}(1)$ & $-156.7(3)$ \\
$\mathrm{C}(2)-\mathrm{C}(1)-\mathrm{C}(5)-\mathrm{O}(1)$ & $84.9(3)$ \\
$\mathrm{N}(1)-\mathrm{C}(4)-\mathrm{C}(7)-\mathrm{C}(12)$ & $114.7(3)$ \\
$\mathrm{C}(3)-\mathrm{C}(4)-\mathrm{C}(7)-\mathrm{C}(12)$ & $-124.1(3)$ \\
$\mathrm{N}(1)-\mathrm{C}(4)-\mathrm{C}(7)-\mathrm{C}(8)$ & $-61.9(4)$ \\
$\mathrm{C}(3)-\mathrm{C}(4)-\mathrm{C}(7)-\mathrm{C}(8)$ & $59.3(4)$ \\
$\mathrm{C}(12)-\mathrm{C}(7)-\mathrm{C}(8)-\mathrm{C}(9)$ & $-0.7(4)$ \\
$\mathrm{C}(4)-\mathrm{C}(7)-\mathrm{C}(8)-\mathrm{C}(9)$ & $176.0(3)$ \\
$\mathrm{C}(7)-\mathrm{C}(8)-\mathrm{C}(9)-\mathrm{C}(10)$ & $0.4(5)$ \\
$\mathrm{C}(8)-\mathrm{C}(9)-\mathrm{C}(10)-\mathrm{C}(11)$ & $-0.2(5)$ \\
$\mathrm{C}(9)-\mathrm{C}(10)-\mathrm{C}(11)-\mathrm{C}(12)$ & $0.2(5)$ \\
$\mathrm{C}(8)-\mathrm{C}(7)-\mathrm{C}(12)-\mathrm{C}(11)$ & $0.7(4)$ \\
$\mathrm{C}(4)-\mathrm{C}(7)-\mathrm{C}(12)-\mathrm{C}(11)$ & $-175.9(3)$ \\
$\mathrm{C}(10)-\mathrm{C}(11)-\mathrm{C}(12)-\mathrm{C}(7)$ & $-0.5(5)$ \\
$\mathrm{O}(3)-\mathrm{S}(1)-\mathrm{C}(13)-\mathrm{C}(18)$ & $151.3(2)$ \\
$\mathrm{O}(4)-\mathrm{S}(1)-\mathrm{C}(13)-\mathrm{C}(18)$ & $21.4(3)$ \\
$\mathrm{C}(3)-\mathrm{S}(1)-\mathrm{C}(13)-\mathrm{C}(18)$ & $-94.3(3)$ \\
$\mathrm{O}(3)-\mathrm{S}(1)-\mathrm{C}(13)-\mathrm{C}(14)$ & \\
& $-27.0(3)$ \\
&
\end{tabular}




$\begin{array}{lc}\mathrm{O}(4)-\mathrm{S}(1)-\mathrm{C}(13)-\mathrm{C}(14) & -156.9(2) \\ \mathrm{C}(3)-\mathrm{S}(1)-\mathrm{C}(13)-\mathrm{C}(14) & 87.4(2) \\ \mathrm{C}(18)-\mathrm{C}(13)-\mathrm{C}(14)-\mathrm{C}(15) & 0.1(5) \\ \mathrm{S}(1)-\mathrm{C}(13)-\mathrm{C}(14)-\mathrm{C}(15) & 178.4(2) \\ \mathrm{C}(13)-\mathrm{C}(14)-\mathrm{C}(15)-\mathrm{C}(16) & 1.0(5) \\ \mathrm{C}(14)-\mathrm{C}(15)-\mathrm{C}(16)-\mathrm{C}(17) & -1.3(5) \\ \mathrm{C}(15)-\mathrm{C}(16)-\mathrm{C}(17)-\mathrm{C}(18) & 0.4(5) \\ \mathrm{C}(14)-\mathrm{C}(13)-\mathrm{C}(18)-\mathrm{C}(17) & -1.0(4) \\ \mathrm{S}(1)-\mathrm{C}(13)-\mathrm{C}(18)-\mathrm{C}(17) & -179.2(2) \\ \mathrm{C}(16)-\mathrm{C}(17)-\mathrm{C}(18)-\mathrm{C}(13) & 0.7(5) \\ \mathrm{C}(5)-\mathrm{C}(1)-\mathrm{N}(1)-\mathrm{C}(4) & -163.9(3) \\ \mathrm{C}(2)-\mathrm{C}(1)-\mathrm{N}(1)-\mathrm{C}(4) & -41.8(3) \\ \mathrm{C}(7)-\mathrm{C}(4)-\mathrm{N}(1)-\mathrm{C}(1) & 160.4(3) \\ \mathrm{C}(3)-\mathrm{C}(4)-\mathrm{N}(1)-\mathrm{C}(1) & 33.1(3) \\ \mathrm{O}(2)-\mathrm{C}(5)-\mathrm{O}(1)-\mathrm{C}(6) & -0.9(5) \\ \mathrm{C}(1)-\mathrm{C}(5)-\mathrm{O}(1)-\mathrm{C}(6) & -179.7(3)\end{array}$

